\title{
Radio Jet Feedback and Star Formation in Heavily Obscured, Hyperluminous Quasars at Redshifts \# 0.5-3. I. Alma Observations
}

\section{Citation}

Lonsdale, Carol J., M. Lacy, A. E. Kimball, A. Blain, M. Whittle, B. Wilkes, D. Stern, et al. 2015. "Radio Jet Feedback and Star Formation in Heavily Obscured, Hyperluminous Quasars at Redshifts \# 0.5-3. I. Alma Observations." The Astrophysical Journal 813 (1) (October 27): 45. doi:10.1088/0004-637x/813/1/45.

\section{Published Version}

doi:10.1088/0004-637X/813/1/45

\section{Permanent link}

http://nrs.harvard.edu/urn-3:HUL.InstRepos:30212130

\section{Terms of Use}

This article was downloaded from Harvard University's DASH repository, and is made available under the terms and conditions applicable to Other Posted Material, as set forth at http:// nrs.harvard.edu/urn-3:HUL.InstRepos:dash.current.terms-of-use\#LAA

\section{Share Your Story}

The Harvard community has made this article openly available.

Please share how this access benefits you. Submit a story. 


\title{
RADIO JET FEEDBACK AND STAR FORMATION IN HEAVILY OBSCURED, HYPERLUMINOUS QUASARS AT REDSHIFTS $\sim 0.5-3$. I. ALMA OBSERVATIONS
}

\author{
Carol J. Lonsdale ${ }^{1}$, M. Lacy ${ }^{1,17}$, A. E. Kimball ${ }^{1,2,17}$, A. Blain ${ }^{3}$, M. Whittle ${ }^{4}$, B. Wilkes ${ }^{5}$, D. Stern ${ }^{6}$, J. Condon ${ }^{1}$, \\ M. $\mathrm{Kim}^{7,8,9}$, R. J. Assef ${ }^{10,17}$, C.-W. Tsai ${ }^{6}$, A. Efstathiou ${ }^{11}$, S. Jones ${ }^{3}$, P. Eisenhardt ${ }^{6}$, C. Bridge ${ }^{12}$, J. Wu ${ }^{13}$, \\ Colin J. Lonsdale ${ }^{14}$, K. Jones ${ }^{4}$, T. Jarrett ${ }^{15}$, And R. Smith ${ }^{16}$ \\ ${ }^{1}$ National Radio Astronomy Observatory, Charlottesville, VA 22903, USA; clonsdal@nrao.edu \\ ${ }^{2}$ CSIRO Astronomy and Space Science, Australia Telescope National Facility, P.O. Box 76, Epping, NSW 1710, Australia \\ ${ }^{3}$ Department of Physics \& Astronomy, University of Leicester, University Road, Leicester LE1 7RH, UK \\ ${ }^{4}$ Department of Astronomy, University of Virginia, Charlottesville, VA 22903, USA \\ ${ }^{5}$ Harvard-Smithsonian Center for Astrophysics, Cambridge, MA 02138, USA \\ ${ }^{6}$ Jet Propulsion Laboratory, California Institute of Technology, Pasadena, CA 91109, USA \\ ${ }^{7}$ The Observatories of the Carnegie Institution for Science, 813 Santa Barbara Street, Pasadena, CA 91101, USA \\ ${ }^{8}$ Korea Astronomy and Space Science Institute, Daejeon 305-348, Korea \\ 9 KASI-Carnegie Fellow, The Observatories of the Carnegie Institution for Science, Pasadena, CA 91101, USA \\ ${ }^{10}$ Núcleo de Astronomía de la Facultad de Ingeniería, Universidad Diego Portales, Av. Ejército Libertador 441, Santiago, Chile; NASA Postdoctoral Program (NPP) \\ ${ }^{11}$ School of Sciences, European University Cyprus, Diogenes Street, Engomi, 1516 Nicosia, Cyprus \\ ${ }^{12}$ California Institute of Technology, 249-17, Pasadena, CA 91125, USA \\ ${ }^{13}$ Division of Astronomy \& Astrophysics, University of California, Los Angeles, Physics and Astronomy Building, 430 Portola Plaza, \\ Los Angeles, CA 90095-1547, USA \\ ${ }^{14}$ Massachusetts Institute of Technology, Haystack Observatory, Westford, MA 01886, USA \\ ${ }^{15}$ Astronomy Department, University of Cape Town, Cape Town, South Africa \\ ${ }^{16}$ Department of Physics, 3141 Chestnut Street, Drexel University, Philadelphia, PA 19104, USA \\ Received 2015 April 7; accepted 2015 August 28; published 2015 October 27
}

\begin{abstract}
We present Atacama Large Millimeter/submillimeter Array (ALMA) $870 \mu \mathrm{m}(345 \mathrm{GHz})$ data for 49 high-redshift $(0.47<z<2.85)$, luminous $\left(11.7<\log \left(L_{\mathrm{bol}} / L_{\odot}\right)<14.2\right)$ radio-powerful active galactic nuclei (AGNs), obtained to constrain cool dust emission from starbursts concurrent with highly obscured radiative-mode black hole $(\mathrm{BH})$ accretion in massive galaxies that possess a small radio jet. The sample was selected from the Wide-field Infrared Survey Explorer with extremely steep (red) mid-infrared colors and with compact radio emission from NVSS/FIRST. Twenty-six sources are detected at $870 \mu \mathrm{m}$, and we find that the sample has large mid- to farinfrared luminosity ratios, consistent with a dominant and highly obscured quasar. The rest-frame $3 \mathrm{GHz}$ radio powers are $24.7<\log \left(P_{3.0 \mathrm{GHz}} / \mathrm{W} \mathrm{Hz}^{-1}\right)<27.3$, and all sources are radio-intermediate or radio-loud. $\mathrm{BH}$ mass estimates are $7.7<\log \left(M_{\mathrm{BH}} / M_{\odot}\right)<10.2$. The rest-frame $1-5 \mu \mathrm{m}$ spectral energy distributions are very similar to the "Hot DOGs" (hot dust-obscured galaxies), and steeper (redder) than almost any other known extragalactic sources. ISM masses estimated for the ALMA-detected sources are $9.9<\log \left(M_{\mathrm{ISM}} / M_{\odot}\right)<11.75$ assuming a dust temperature of $30 \mathrm{~K}$. The cool dust emission is consistent with star formation rates reaching several thousand $M_{\odot}$ $\mathrm{yr}^{-1}$, depending on the assumed dust temperature, but we cannot rule out the alternative that the AGN powers all the emission in some cases. Our best constrained source has radiative transfer solutions with approximately equal contributions from an obscured AGN and a young (10-15 Myr) compact starburst.
\end{abstract}

Key words: galaxies: active - galaxies: evolution - galaxies: jets - quasars: general - radio continuum: galaxies submillimeter: galaxies

\section{INTRODUCTION}

Central questions concerning coeval galaxy and supermassive black hole (SMBH) evolution include the relative timescales and mechanisms for stellar mass and black hole (BH) mass building including the roles and duty cycles of (a) secular versus merger-triggered mechanisms for driving material into the central regions; (b) "radiative-mode" versus "jet-mode" BH accretion modes and rates; and (c) "quasarmode" versus "radio-mode" feedback mechanisms, all as a function of epoch, galaxy mass, and galaxy environment. Jetmode active galactic nuclei (AGNs) are low-excitation systems in which the AGN is powered by advection-dominated accretion flows onto the $\mathrm{BH}$ with a low accretion rate, as recently reviewed by Heckman \& Best (2014). AGNs in jet

\footnotetext{
${ }^{17}$ Visiting Astronomer, Cerro Tololo Inter-American Observatory. CTIO is operated by AURA, Inc., under contract to the National Science Foundation.
}

mode are expected to have low radiative emission and to be energetically dominated by the jet kinetic outflow. Jet-mode radio AGNs are thought to be highly effective in maintaining galaxies free of new gas and star formation via "radio-mode" kinetic feedback, i.e. jet inflation of bubbles in the surrounding hot intergalactic gas (Croton 2006; Cattaneo \& Teyssier 2007; Fabian 2010). Radiative-mode, or "quasar-mode," AGNs have higher accretion rates from a thin accretion disk whose radiation powers the narrow- and broad-line regions, and which is fed through a surrounding dusty torus or "torus-like" structure. Quasar-mode accretion has a lower duty cycle than jet-mode, occurring when large amounts of material are available for accretion onto the SMBH. Quasar-mode AGNs are capable of powering efficient feedback into the host galaxy via thermal winds from the accretion disk, disrupting star formation and ejecting gas.

Powerful jets are also found in $\sim 10 \%$ of radiatively efficient AGNs, the radio-loud QSOs (also known as broad-line radio 
galaxies, BLRGs, and high excitation radio galaxies, HERGs), and therefore jets may also contribute to feedback activity in quasar-mode AGNs (Holt et al. 2008; Nesvadba et al. 2008). It is often assumed that the high-radio-power Fanaroff-Riley type II (FRII) jets are too collimated to impact the ISM within host galaxies (De Young 2010). However, high-resolution hydrodynamic models by Wagner \& Bicknell (2011) and Wagner et al. $(2012,2013)$ have demonstrated that the impact of powerful jets on the ISM within the central regions of AGN host galaxies is highly dependent on the density, the filling factor, and the average size of the cool clouds within the ISM. High porosity leads to the inflation of a cocoon by the overpressured jet, leading to a quasispherical bubble being driven into the ISM. Wagner \& Bicknell (2011) and Wagner et al. (2012) find that jets of moderate to high power can accelerate dense ISM gas from a hundred to several thousand $\mathrm{km} \mathrm{s}^{-1}$, with wide-angle flows, within 10-100 Myr.

Best \& Heckman (2012) and Best et al. (2014) have shown that the two populations of radio AGNs, quasar-mode (or radiative-mode) and radio-mode (or jet-mode), are both found across all radio luminosities, and that the radiative-mode radio AGNs show much stronger evolution with cosmic time than jet-mode radio AGN, with an order-of-magnitude increase in space density out to $z \sim 1$. This evolution in space density is similar to that of the star formation rate (SFR) density and the quasar luminosity function, consistent with the scenario that the radiative-mode AGNs are controlled by episodic cold gas supply.

Gas flows associated with gas-rich mergers are the likely source of episodic cold gas fueling, important for building galaxies and BHs by triggering both starbursts and AGN activity. Morphological signatures of mergers have been found in a large faction of powerful, $z<0.7$, radio galaxies (RGs) which display spectroscopic signatures of young stellar populations (Tadhunter et al. 2011), and those with the youngest stellar populations $(<0.1 \mathrm{Gyr})$ show a correlation with mid- to far-infrared and [O III] luminosity, indicating the presence of a radiative-mode AGN. Tadhunter et al. (2011) also find significant complexity in correlations between the triggering or re-triggering of jets, recent star formation, and the merging of the dual nuclei, implying chaotic gas infall histories during merger events, while Dicken et al. (2011) find evidence for the strongest correlation between recent star formation and radio jets for the radio AGNs with the most compact jets. These results support the idea that small (young) radio jets play an important role in the evolution of massive galaxies and SMBHs during merger-driven phases of high accretion rate.

In this series of papers we address the impact of young, moderate to powerful, radio jets from luminous, radiatively efficient, highly obscured, radio AGNs on the disruption of the ISM and star formation in their hosts at redshifts near the peak of galaxy and $\mathrm{BH}$ building, $z \sim 1-3$. We also consider the possibility of ISM compression and starburst triggering by jet kinetic energy. By selecting systems with a high mid-infrared (MIR) luminosity we aim to identify radiatively efficient AGNs, and by selecting compact radio sources we favor young radio jets that are confined within the hosts. By selecting AGNs that are very red through the optical-MIR we favor highly obscured systems likely to be in a peak fueling phase.

A nearby example of such a system is the MIR-bright QSO Mrk 231, which has a luminous radiative-mode AGN, two small radio jet systems ( 2 and 40 pc) (Ulvestad et al. 1999; Lonsdale et al. 2003), and powerful molecular outflows (Fischer et al. 2010; Gonzalez-Alfonso et al. 2014; Aalto et al. 2015; Feruglio et al. 2015). Mrk 231 also shows a bright optical core, indicating a complex nuclear geometry.

\subsection{Evidence for Radio Jet Interactions with Molecular Gas}

Most early studies of outflows from AGNs targeted the ionized gas, which, because of its much lower mass, requires much less energy to disperse than the neutral and molecular gas. More recent work shows that jet-induced feedback can indeed impact both warm and cold molecular gas in RGs (Feruglio et al. 2010, 2015; Fischer et al. 2010; Sturm et al. 2011; Dasyra \& Combes 2012; Combes et al. 2013; Morganti et al. 2013; Dasyra et al. 2014; Garca-Burillo et al. 2014; Gonzalez-Alfonso et al. 2014; Tadhunter et al. 2014a, 2014b), including turbulence and shock-excited $\mathrm{H}_{2}$ emission in RGs (e.g., Morganti et al. 2003; Nesvadba et al. 2008, 2011a, 2011b; Guillard et al. 2012, 2015). Molecular hydrogen emission galaxies have large mid-IR $\mathrm{H}_{2}$ luminosities that are too high for photodissociation regions, and which are most likely generated by jet-generated shocks in the ISM (Appleton et al. 2006; Ogle et al. 2010; Guillard et al. 2012; Lanz et al. 2015). Most of these RGs are in a phase of radio-mode accretion without strong evidence for a concurrent radiative-mode AGN core. Quasar winds may also contribute to molecular outflows in radiative-mode radio AGNs (e.g., Veilleux et al. 2013), such as those we study here; therefore our sample is ideal for studying the relative importance of these two feedback modes in early feedback phases of heavily obscured objects.

\subsection{MIR-selected Highly Obscured Quasars}

A rare class of highly obscured and luminous quasars at redshifts above 1 was revealed in follow-up studies of extremely red (from the optical to the MIR) sources found in Spitzer surveys (Lutz et al. 2005; Yan et al. 2005; Polletta et al. 2006; Dey et al. 2008; Lacy et al. 2011). These systems can have luminosities over $10^{13} L_{\odot}$, and deep X-ray observations have revealed Compton-thick AGNs in some $\left(N_{\mathrm{H}}>1.5 \times\right.$ $10^{24} \mathrm{~cm}^{-2}$; Polletta et al. 2008). The reddest of these Spitzerselected systems are sometimes referred to as dust-obscured galaxies, "DOGs" (Dey et al. 2008).

The Wide-field Infrared Survey Explorer (WISE), which covers the entire sky at 3.4, 4.6, 12, and $22 \mu \mathrm{m}$ (henceforth refereed to as the $W 1, W 2, W 3$, and $W 4$ bands) (Wright et al. 2010; Jarrett et al. 2011; Cutri et al. 2012), has opened up the entire MIR sky to searches for obscured QSOs by their MIR signatures (Eisenhardt et al. 2012; Stern et al. 2012, 2014; Wu et al. 2012, 2014; Assef et al. 2013; Bridge et al. 2013; Yan et al. 2013; Jones et al. 2014; Tsai et al. 2015). Although not as deep as the largest Spitzer surveys (e.g., Lonsdale et al. 2004; Ashby et al. 2009, 2013), WISE is sensitive enough to see the dust thermal emission from the most powerful quasars to redshifts $>4$. The first results from WISE follow-up of the reddest sources (selected without regard to radio brightness) have indeed revealed an extremely IR-luminous population of high-redshift quasars, exceeding $10^{14} L_{\odot}$ in bolometric luminosity in some cases (Eisenhardt et al. 2012; Wu et al. 2012, 2014; Bridge et al. 2013; Jones et al. 2014; Assef et al. 2015; Tsai et al. 2015). Their bolometric luminosities are MIR- 
dominated, which led $\mathrm{Wu}$ et al. (2012) to dub them "Hot DOGs" (hot dust-obscured galaxies), while Bridge et al. (2013) investigate Ly $\alpha$ Blobs discovered around a high percentage of their red WISE sample, the WISE Lyman alpha Blobs (WLABs). X-ray observations show only faint fluxes, consistent with highly obscured X-ray AGNs (Stern et al. 2014; Pinconcelli et al. 2015). In this paper we will henceforth refer to the main discovery papers for these WISE Hot DOGs and WLABs (Eisenhardt et al. 2012; Wu et al. 2012; Bridge et al. 2013) as EWB12.

\subsection{This Work}

We present a snapshot survey of 49 sources with the Atacama Large Millimeter/submillimeter Array, ALMA, at $870 \mu \mathrm{m}$, which represents a southern sky subset of our sample of 156 radio-powerful (RP) obscured quasar candidates. The sample has been selected to be unresolved in the NVSS and FIRST radio surveys (Becker et al. 1995; Condon et al. 1998) and ultra-red in the WISE MIR survey, with similar selection criteria as the Hot DOGs (EWB12), to search for radio-jet dominated feedback from massive, obscured quasars. We also present redshifts obtained from optical and/or near-IR (NIR) spectroscopy for 45 of these ALMA-observed quasars, and additional FIR-submillimeter photometry from other facilities.

The sample is described in Section 2 and the observations in Section 3. The results are presented in Section 4, followed by spectral energy distribution (SED) model fitting and derivation of luminosities and masses in Section 5. The discussion is in Section 6 and conclusions in Section 7. Seven sources with NIR spectroscopy from FIRE on Magellan have been discussed by Kim et al. (2013). Jones et al. (2015) have published deep SCUBA $850 \mu \mathrm{m}$ imaging from the James Clerk Maxwell Telescope (JCMT) for 30 northern sources from our overall sample of 156 RP quasars, detecting four, and finding an excess of serendipitously detected $850 \mu \mathrm{m}$ sources in the fields on $\sim 1 \mathrm{Mpc}$ scales. Silva et al. (2015) have found an excess of serendipitously detected $870 \mu \mathrm{m}$ sources in the 49 ALMA images discussed here, in agreement with Jones et al. (2015), although on smaller physical size scales of $\sim 150 \mathrm{kpc}$, perhaps indicating an excess of starbursting submillimeter galaxies (SMGs) in the fields of these QSOs. Subsequent papers will address Jansky VLA $8-12 \mathrm{GHz}$ imaging of the full sample (Carol J. Lonsdale et al. 2015, in preparation) and VLBA $C$ band imaging of 90 sources, including 33 from the ALMA sample (Colin J. Lonsdale et al. 2015, in preparation). NIR $J$ and $K_{s}$ imaging for a subset of the ALMA sample from Very Large Telescope (VLT)/ISAAC and VLT/XShooter spectroscopy will be presented in A. Blain et al. (2015, in preparation). We adopt a cosmology with $H_{0}=71 \mathrm{~km} \mathrm{~s}^{-1} \mathrm{Mpc}^{-1}$, $\Omega_{\Lambda}=0.73$, and $\Omega_{M}=0.27$.

\section{SAMPLE SELECTION}

For our overall sample of ultra-red, radio-powerful sources, we cross-matched the NVSS catalog, which covers the sky north of $\delta=-40^{\circ}$, with point sources from the WISE Allsky Catalog. The ALMA-observed subsample described here was selected at an earlier time when only the WISE Preliminary Catalog was available, as explained further below. Extended WISE sources were rejected based on the cataloged ext flag. We used positional information from the higher resolution but smaller area FIRST catalog where available, and excluded

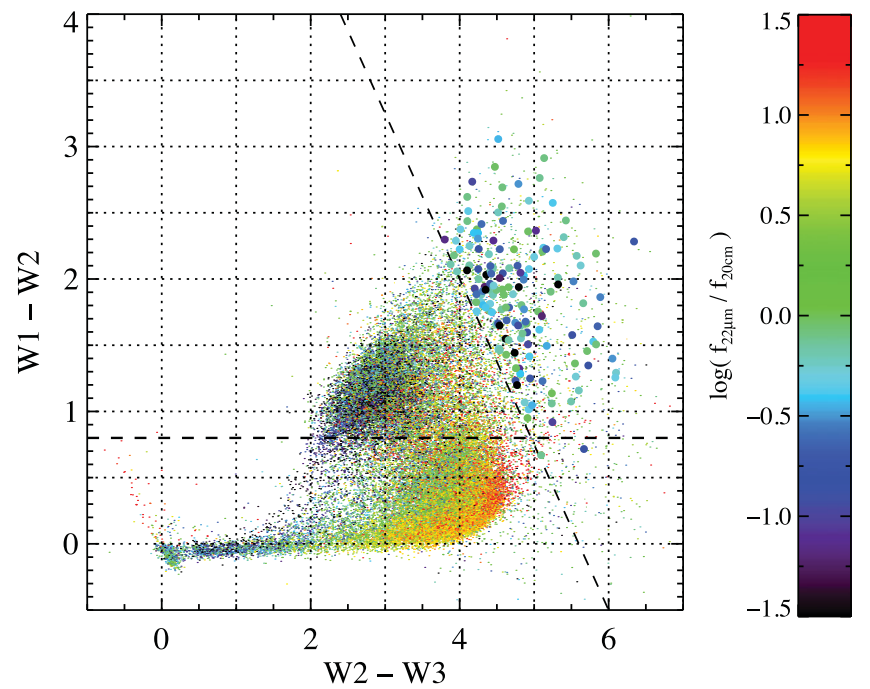

Figure 1. WISE 3.4-4.6-12 $\mu \mathrm{m}(W 1-W 2-\mathrm{W} 3$ ) color-color plot (using Vega magnitudes) showing the full WISE-NVSS sample of 54,457 sources, colorcoded by radio loudness (see Figure 2) as shown by the color bar on the right, radio loudness increasing red to black. The sequence at the bottom is the sequence of normal spirals and starbursts. The flux-limited ultra-red sample of 156 sources is highlighted above the dashed line with larger symbols. The horizontal dashed line shows the AGN color selection criterion used by Stern et al. (2012): $(W 1-W 2)>0.8$, for comparison to our ultra-red selection criterion.

$\pm 10^{\circ}$ from the Galactic plane. Within this area of 28,443 square degrees of mutual NVSS-WISE coverage are 54,457 WISE sources that have a robust point source detection (SNR > 7) in the WISE $12 \mu \mathrm{m}$ and/or $22 \mu \mathrm{m}$ bands and an NVSS/FIRST $1.4 \mathrm{GHz}$ match with a separation of $<7^{\prime \prime}$, the best compromise between completeness and reliability based on a randomized match analysis. The entire sample is illustrated in the WISE 3.4-4.6-12 $\mu \mathrm{m}$ color-color diagram in Figure 1, where we plot the WISE-NVSS sample color-coded by $q_{22}=\log \left(f_{22 \mu \mathrm{m}} / f_{20 \mathrm{~cm}}\right)$, the $22 \mu \mathrm{m} q$ parameter, which is a measure of radio loudness (Appleton et al. 2004; Ivison et al. 2007; Ibar et al. 2008). The sequence of low-redshift normal spirals and starbursts has blue $(W 1-W 2)$ colors (where $W X$ is the Vega magnitude in WISE band number $\mathrm{X}$ ) with a wide range of $(W 2-W 3)$ colors, and a cloud of AGNs is seen with redder (W1 - W2) colors (Wright et al. 2010; Jarrett et al. 2011; Yan et al. 2013). The most radio-loud systems are seen toward the left of each of the normal galaxy sequences and the AGN cloud, consisting of the radio-mode and the radiative-mode AGNs, respectively. For low-redshift galaxies the point source fluxes used for this figure may underestimate the total fluxes.

Highly obscured luminous AGNs are expected to be among the reddest sources in this figure. Previous authors have used color cuts in MIR color space and/or MIR-optical space to select the reddest extragalactic Spitzer and WISE sources (e.g., Dey et al. 2008; EWB12). We have instead chosen to reduce potential bias caused by color cuts, due to the complexity of the observed MIR spectral shape of these sources, which depends strongly on redshift due to the polycyclic aromatic hydrocarbon (PAH) emission features and the 9.7 and $18 \mu \mathrm{m}$ silicate features. Therefore we have included all sources lying significantly redward of the main WISE populations. This is illustrated in Figure 1 by the dashed line, defined empirically as $(W 2-W 3)+1.25(W 1-W 2)>7$. The resulting number of sources in the sample redward of our MIR selection threshold 


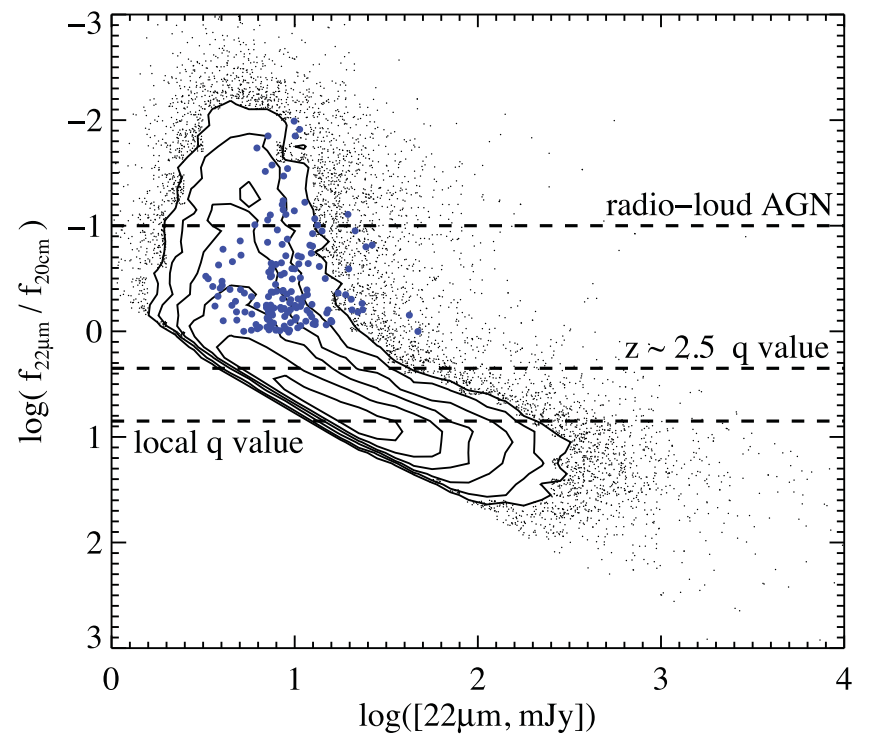

Figure 2. The $22 \mu \mathrm{m} q$ value, $q_{22}=\log \left(f_{22 \mu \mathrm{m}} / f_{20 \mathrm{~cm}}\right)$ vs. WISE $22 \mu \mathrm{m}$ flux density, for the 156 ultra-red, radio-powerful sources (blue points), compared to the full WISE/NVSS cross-matched sample of 54,457 sources (dots and contours). The completeness limit of the NVSS is responsible for the diagonal cutoff at the lower left. Also shown are the mean expected values for starforming galaxies of the infrared-to-radio parameter, $q_{24}=\log \left(f_{24 \mu \mathrm{m}} / f_{20 \mathrm{~cm}}\right)$ for the Spitzer $24 \mu \mathrm{m}$ band by Ibar et al. (2008) in the local universe and the $k$ corrected $z \sim 2.5$ value. The representative radio-loud SED from Elvis et al. (1994) is also shown. The subset of ultra-red sources observed with ALMA was selected to have $0>\log \left(f_{22 \mu \mathrm{m}} / f_{22 \mathrm{~cm}}\right)>-1$.

in Figure 1 is 1858 . To reject radio-quiet systems we added the requirement that $\log \left(f_{22} \mu \mathrm{m} / f_{20 \mathrm{~cm}}\right)<0$ as illustrated in Figure 2. For comparison, the color selection criteria for the Hot DOGs of Eisenhardt et al. (2012) and Wu et al. (2012) are either $(W 2-W 4)>8.2$ or $(W 2-W 3)>5.3$, termed by these authors the "W1W2drop" criteria. Bridge et al. (2013) have used a slightly different color selection method: $(W 2-W 3) \geqslant$ 4.8. Both studies also use brightness or SNR threshold similar to ours, and neither has a radio flux density criterion.

We inspected all candidates in WISE and DSS images, and Sloan Digital Sky Survey (SDSS) images where available, rejecting low-redshift galaxies, artifacts, and confused sources. To avoid rejecting galaxies or quasars that could plausibly be within the redshift range of interest, $1<z<3$, no specific cut in optical magnitude or MIR/optical flux ratio was imposed. Galaxies with a size or brightness large enough to place them at $z<0.5$ were rejected, while compact sources with high surface brightness were retained to brighter limits as potential highredshift quasars. We are obtaining spectra to reject low-redshift sources from our final sample. Thus, our selection can include objects that are less red (in $R-[22]$ ) than the criterion $(R-[24])>14$ used for Spitzer DOGs (Dey et al. 2008) and similar samples, and, for example, may include systems in which a massive galaxy dominates the optical light. A total of 708 of the NVSS-WISE sample satisfy all our criteria, or $\sim 1.3 \%$ of the entire NVSS-WISE matched sample. Of the 708 sources, 703 (269) are detected at 12 (22) $\mu \mathrm{m}$ with $\mathrm{SNR}>7$.

Due to the strongly varying WISE sensitivity across the sky, caused by the varying coverage level, a flux density threshold at $22 \mu \mathrm{m}$ was applied. For the subsample observed with ALMA the flux density threshold chosen was $4 \mathrm{mJy}$. This was later revised upward to $7 \mathrm{mJy}$ for the remainder of the sample when the Allsky Catalog became available. The final sample is 156 sources, 49 of which belong to the ALMA-observed subsample.

The ALMA subsample fluxes have been revised in the updated WISE Allsky Catalog, some now falling below the original $4 \mathrm{mJy}$ threshold. These have been retained in the sample. The decl. range for the ALMA subsample was chosen to allow access to many northern facilities as well as ALMA: $-40^{\circ}<\delta<+1^{\circ}$. The ALMA sample is restricted in R.A. to two regions: $3^{\mathrm{h}}<$ R. A. $<8^{\mathrm{h}} 30^{\mathrm{m}}$ and $13^{\mathrm{h}}<$ R. A. $<21^{\mathrm{h}}$, due to the incompleteness of the WISE Preliminary Catalog at that time. Fifty-five sources met our original selection criteria within these areas. Six of these have $\log \left(f_{22 \mu \mathrm{m}} / f_{20 \mathrm{~cm}}\right)<-1$ and were excluded to disfavor classical double-lobed sources, a criterion that was later dropped for the full sample of 156 sources. Of the final ALMA subsample of 49 sources, 48 (23) have SNR $>7$ at 12 (22) $\mu \mathrm{m}$ in the WISE Allsky Catalog, and the minimum $12 / 22 \mu \mathrm{m}$ SNR is $6.7 / 2.6$. Only one source fails the original SNR criterion of SNR $>7$ at 12 or $22 \mu \mathrm{m}$ after the Allsky flux revision: WISE J204049.51-390400.5, which has an SNR at $12 / 22 \mu \mathrm{m}$ of $6.7 / 6.2$. Due to the evolving selection criteria the sample is not complete to a fixed SNR or flux density limit.

\section{OBSERVATIONS}

\subsection{FIR and Submillimeter Observations}

Twenty-three sources were observed with ALMA in two Band 7 scheduling blocks on 2011 November 16. An additional 14 were observed on 2012 May 25 and the final 12 on 2012 August 29. The central frequency was set to $345 \mathrm{GHz}(870 \mu \mathrm{m})$, with an $8 \mathrm{GHz}$ bandwidth split into two sidebands. Fifteen antennas were used in the "compact" Early Science configuration for the 2011 November observations, 19 for the 2012 May observations, and 23 for the August observations. The resulting beamsizes are 1 !! 2,0 !" 5 , and 1 !! 2 , respectively.

For the November observations, Callisto was used for flux calibration. Titan was used for seven sources in May and Neptune for the other seven sources in May. Titan was again used for all sources in August. The objects were observed with the correlator in time division mode, which results in 256 channels per sideband with a spectral resolution of $14 \mathrm{~km} \mathrm{~s}^{-1}$. Time on source was 1.5 minute per object, resulting in an rms noise of $0.3-0.6 \mathrm{mJy}$; the lower noise levels generally correspond to the observations with larger antenna numbers. Flux densities were measured using imfit in CASA, and are reported in Table 1.

Four sources from our overall sample of 156 were observed at $350 \mu \mathrm{m}$ at the Submillimeter High Angular Resolution Camera II installed at the $10.4 \mathrm{~m}$ Caltech Submillimeter Observatory (CSO) telescope (Dowell et al. 2003) on UT 2012 March 22. Two of these, W1343-1136 and W1400-2919, belong to the ALMA subsample. Our observing and data reduction process follows that described by Wu et al. (2012).

Seventy-seven of our full sample of 156 sources, including all the ALMA targets, were queued for Herschel ${ }^{18}$ PACS (Poglitsch et al. 2010) and SPIRE (Griffin et al. 2010) photometric observations (PID: OT2_clonsdal_1). In total 15 objects were observed by PACS, and five with SPIRE, before

\footnotetext{
18 Herschel is an ESA space observatory with science instruments provided by European-led Principal Investigator consortia and with important participation from NASA.
} 
Table 1

Optical, WISE, NVSS, and ALMA Photometry

\begin{tabular}{|c|c|c|c|c|c|c|c|c|c|}
\hline WISE Name & $\begin{array}{c}R \text {-band } \\
\text { app. mag. } \\
\text { (Vega) }\end{array}$ & $\begin{array}{c}f_{3.4 \mu \mathrm{m}} \\
(\mathrm{mJy}) \\
W I S E \\
\end{array}$ & $\begin{array}{c}f_{4.6 \mu \mathrm{m}} \\
(\mathrm{mJy}) \\
W I S E \\
\end{array}$ & $\begin{array}{l}f_{12 \mu \mathrm{m}} \\
(\mathrm{mJy}) \\
\text { WISE } \\
\end{array}$ & $\begin{array}{l}f_{22 \mu \mathrm{m}} \\
(\mathrm{mJy}) \\
W I S E \\
\end{array}$ & $\begin{array}{c}q_{22} \\
\text { Observed } \\
\text { frame } \\
\end{array}$ & $\begin{array}{c}f_{1.4 \mathrm{GHz}} \\
(\mathrm{mJy}) \\
\mathrm{NVSS} \\
\end{array}$ & 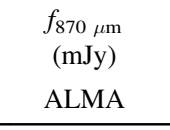 & $\begin{array}{c}\log \\
\left(f_{870 \mu \mathrm{m}} /\right. \\
\left.f_{22 \mu \mathrm{m}}\right) \\
\end{array}$ \\
\hline J030427.53-310838 & $22.0 \pm 0.3$ & $0.179 \pm 0.0066$ & $0.693 \pm 0.018$ & $4.55 \pm 0.12$ & $10.87 \pm 0.65$ & -0.70 & $54.99 \pm 1.72$ & $2.8 \pm 0.3$ & -0.52 \\
\hline J030629.21-335332 & $20.7 \pm 0.2$ & $0.194 \pm 0.0070$ & $0.749 \pm 0.020$ & $6.19 \pm 0.14$ & $4.83 \pm 0.65$ & -0.11 & $6.17 \pm 0.52$ & $1.7 \pm 0.4$ & -0.45 \\
\hline J035448.24-330827 & $22.5 \pm 0.2$ & $0.086 \pm 0.0063$ & $0.314 \pm 0.015$ & $3.34 \pm 0.12$ & $7.05 \pm 0.78$ & -0.04 & $7.73 \pm 0.54$ & $<1.2$ & $<-1.12$ \\
\hline J040403.61-243600 & $20.7 \pm 0.2$ & $0.104 \pm 0.0072$ & $0.326 \pm 0.017$ & $4.63 \pm 0.15$ & $4.43 \pm 0.92$ & -0.40 & $11.04 \pm 0.58$ & $3.1 \pm 0.4$ & -0.16 \\
\hline J040937.67-183757 & $21.3 \pm 0.2$ & $0.517 \pm 0.0147$ & $2.492 \pm 0.054$ & $22.97 \pm 0.36$ & $47.07 \pm 1.68$ & 0.00 & $46.91 \pm 1.48$ & $<1.5$ & $<-1.47$ \\
\hline J041754.10-281654 & $23.9 \pm 0.3$ & $0.043 \pm 0.0057$ & $0.143 \pm 0.013$ & $1.43 \pm 0.10$ & $3.84 \pm 0.85$ & -0.63 & $16.32 \pm 0.68$ & $4.3 \pm 0.6$ & 0.05 \\
\hline J043921.92-315908 & $24.2 \pm 0.3$ & $0.034 \pm 0.0051$ & $0.090 \pm 0.011$ & $1.19 \pm 0.09$ & $4.57 \pm 0.77$ & -0.66 & $20.88 \pm 0.78$ & $6 \pm 0.4$ & 0.12 \\
\hline J051905.84-081320 & $>24$ & $0.059 \pm 0.0071$ & $0.136 \pm 0.015$ & $1.76 \pm 0.13$ & $5.09 \pm 0.85$ & -0.72 & $26.79 \pm 0.90$ & $<1.5$ & $<-0.56$ \\
\hline J052533.47-361440 & $22.9 \pm 0.3$ & $0.013 \pm 0.0053$ & $0.046 \pm 0.010$ & $1.38 \pm 0.10$ & $4.07 \pm 0.71$ & -0.10 & $5.12 \pm 0.56$ & $<1.5$ & $<-0.41$ \\
\hline J052624.72-322500 & $22.6 \pm 0.2$ & $0.023 \pm 0.0056$ & $0.106 \pm 0.011$ & $6.73 \pm 0.17$ & $26.47 \pm 1.17$ & -0.82 & $173.5 \pm 5.23$ & $18 \pm 0.5$ & -0.17 \\
\hline J053622.59-270300 & $23.2 \pm 0.3$ & $0.065 \pm 0.0060$ & $0.404 \pm 0.016$ & $3.26 \pm 0.12$ & $5.35 \pm 0.80$ & -0.18 & $8.16 \pm 0.56$ & $2.7 \pm 0.4$ & -0.30 \\
\hline J054930.07-373939 & $>24$ & $0.021 \pm 0.0049$ & $0.091 \pm 0.010$ & $1.09 \pm 0.09$ & $3.37 \pm 0.73$ & -0.50 & $10.60 \pm 0.57$ & $2 \pm 0.4$ & -0.23 \\
\hline J061200.23-062209 & $20.8 \pm 0.2$ & $0.330 \pm 0.0118$ & $0.621 \pm 0.022$ & $9.52 \pm 0.20$ & $20.55 \pm 1.01$ & -0.20 & $32.79 \pm 1.08$ & $2.7 \pm 0.6$ & -0.88 \\
\hline J061348.08-340728 & $>24.6$ & $0.065 \pm 0.0065$ & $0.211 \pm 0.012$ & $2.62 \pm 0.11$ & $7.37 \pm 0.71$ & -0.52 & $24.15 \pm 1.16$ & $<1.8$ & $<-0.62$ \\
\hline J061405.57-093658 & $24.1 \pm 0.3$ & $0.017 \pm 0.0073$ & $0.069 \pm 0.015$ & $2.16 \pm 0.14$ & $5.80 \pm 0.84$ & -0.01 & $5.94 \pm 0.51$ & $<1.8$ & $<-0.51$ \\
\hline J063027.81-212058 & $22.2 \pm 0.2$ & $0.020 \pm 0.0070$ & $0.063 \pm 0.014$ & $2.62 \pm 0.13$ & $5.06 \pm 0.90$ & -0.39 & $12.55 \pm 0.60$ & $5 \pm 1.3$ & -0.01 \\
\hline J064228.92-272801 & $21.4 \pm 0.2$ & $0.038 \pm 0.0070$ & $0.108 \pm 0.014$ & $1.33 \pm 0.16$ & $3.67 \pm 0.87$ & -0.24 & $6.36 \pm 0.52$ & $2.2 \pm 0.6$ & -0.22 \\
\hline J065215.85-200612 & $>23.7$ & $<0.013$ & $0.045 \pm 0.013$ & $1.92 \pm 0.13$ & $4.81 \pm 0.83$ & -0.18 & $7.35 \pm 0.52$ & $3.2 \pm 0.5$ & 0.18 \\
\hline J070257.20-280842 & $21.8 \pm 0.2$ & $0.027 \pm 0.0061$ & $0.140 \pm 0.016$ & $1.76 \pm 0.12$ & $4.54 \pm 0.86$ & -0.25 & $8.00 \pm 0.58$ & $<1.8$ & $<-0.40$ \\
\hline J071433.54-363552 & $22.8 \pm 0.2$ & $<0.012$ & $0.039 \pm 0.011$ & $0.99 \pm 0.12$ & $4.01 \pm 0.84$ & -0.47 & $11.95 \pm 0.60$ & $2.4 \pm 0.3$ & -0.22 \\
\hline J071912.58-334944 & $24.1 \pm 0.3$ & $<0.011$ & $0.081 \pm 0.012$ & $1.93 \pm 0.12$ & $4.06 \pm 0.88$ & -0.78 & $24.30 \pm 0.87$ & $5.2 \pm 0.6$ & 0.11 \\
\hline J081131.61-222522 & $21.5 \pm 0.2$ & $0.132 \pm 0.0086$ & $0.611 \pm 0.023$ & $5.62 \pm 0.17$ & $7.61 \pm 1.17$ & -0.37 & $17.84 \pm 0.71$ & $<1.8$ & $<-0.63$ \\
\hline J082311.24-062408 & $22.32 \pm 0.16^{\mathrm{a}}$ & $0.118 \pm 0.0078$ & $0.441 \pm 0.019$ & $4.08 \pm 0.15$ & $10.42 \pm 0.97$ & -0.71 & $53.99 \pm 1.67$ & $<1.8$ & $<-0.76$ \\
\hline J130817.00-344754 & $22.3 \pm 0.2$ & $0.086 \pm 0.0056$ & $0.248 \pm 0.013$ & $3.36 \pm 0.12$ & $9.12 \pm 0.73$ & -0.87 & $68.10 \pm 2.10$ & $1.38 \pm 0.34$ & -0.81 \\
\hline J134331.37-113609 & $21.7 \pm 0.2$ & $0.024 \pm 0.0057$ & $0.136 \pm 0.013$ & $1.61 \pm 0.12$ & $3.81 \pm 0.79$ & -0.33 & $8.18 \pm 0.54$ & $2.34 \pm 0.31$ & -0.22 \\
\hline J140050.13-291924 & $21.7 \pm 0.2$ & $0.110 \pm 0.0063$ & $0.501 \pm 0.018$ & $5.58 \pm 0.14$ & $11.85 \pm 0.77$ & -0.64 & $51.92 \pm 1.63$ & $<0.90$ & $<-1.37$ \\
\hline J141243.15-202011 & $\cdots$ & $0.092 \pm 0.0063$ & $0.333 \pm 0.015$ & $3.39 \pm 0.13$ & $7.41 \pm 0.78$ & -0.09 & $9.01 \pm 0.55$ & $2.55 \pm 0.63$ & -0.45 \\
\hline J143419.59-023543 & $22.02 \pm 0.18^{\mathrm{a}}$ & $0.058 \pm 0.0056$ & $0.257 \pm 0.014$ & $2.13 \pm 0.11$ & $5.04 \pm 0.71$ & -0.86 & $36.15 \pm 1.16$ & $<0.9$ & $<-0.75$ \\
\hline J143931.76-372523 & $\cdots$ & $0.027 \pm 0.0071$ & $0.115 \pm 0.013$ & $2.34 \pm 0.12$ & $3.92 \pm 0.83$ & -0.41 & $10.02 \pm 0.57$ & $<0.6$ & $<-0.75$ \\
\hline J150048.73-064939 & $\cdots$ & $0.068 \pm 0.0065$ & $0.293 \pm 0.016$ & $6.26 \pm 0.17$ & $15.77 \pm 0.94$ & -0.10 & $20.01 \pm 0.73$ & $6.11 \pm 0.28$ & -0.41 \\
\hline J151003.71-220311 & $\cdots$ & $0.143 \pm 0.0095$ & $0.411 \pm 0.020$ & $5.34 \pm 0.18$ & $14.87 \pm 1.09$ & -0.06 & $17.14 \pm 0.70$ & $<0.9$ & $<-1.27$ \\
\hline J151310.42-221004 & $\ldots$ & $0.037 \pm 0.0082$ & $0.214 \pm 0.018$ & $2.64 \pm 0.16$ & $9.71 \pm 1.10$ & -0.50 & $30.40 \pm 1.03$ & $4.86 \pm 0.27$ & -0.30 \\
\hline J151424.12-341100 & $\ldots$ & $0.076 \pm 0.0091$ & $0.184 \pm 0.019$ & $3.12 \pm 0.16$ & $7.01 \pm 1.03$ & -0.25 & $12.39 \pm 0.60$ & $<0.9$ & $<-0.94$ \\
\hline $\mathrm{J} 152116.59+001755$ & $\ldots$ & $0.039 \pm 0.0046$ & $0.274 \pm 0.014$ & $5.41 \pm 0.15$ & $9.51 \pm 0.70$ & -0.60 & $37.89 \pm 1.20$ & $1.19 \pm 0.28$ & -0.90 \\
\hline J154141.64-114409 & $\ldots$ & $0.032 \pm 0.0077$ & $0.155 \pm 0.017$ & $2.91 \pm 0.16$ & $10.74 \pm 1.14$ & -0.51 & $34.52 \pm 1.13$ & $1.2 \pm 0.3$ & -1.11 \\
\hline J163426.87-172139 & $\ldots$ & $0.039 \pm 0.0094$ & $0.101 \pm 0.018$ & $1.70 \pm 0.17$ & $3.57 \pm 1.15$ & -0.42 & $9.49 \pm 0.55$ & $<0.84$ & $<-0.63$ \\
\hline J164107.22-054827 & $\ldots$ & $0.086 \pm 0.0083$ & $0.423 \pm 0.020$ & $3.14 \pm 0.15$ & $6.26 \pm 0.89$ & -0.02 & $6.62 \pm 0.48$ & $2.3 \pm 0.29$ & -0.43 \\
\hline J165305.40-010230 & $\ldots$ & $0.083 \pm 0.0074$ & $0.191 \pm 0.015$ & $2.56 \pm 0.14$ & $5.31 \pm 0.93$ & -0.36 & $12.21 \pm 0.56$ & $<0.78$ & $<-0.83$ \\
\hline J165742.88-174049 & $\ldots$ & $0.073 \pm 0.0102$ & $0.186 \pm 0.026$ & $2.82 \pm 0.24$ & $8.60 \pm 1.01$ & -0.31 & $17.48 \pm 0.71$ & $<0.78$ & $<-1.04$ \\
\hline J170204.65-081108 & $\ldots$ & $0.021 \pm 0.0690$ & $0.074 \pm 0.053$ & $3.05 \pm 0.26$ & $12.32 \pm 1.40$ & -0.74 & $67.59 \pm 2.07$ & $<1.02$ & $<-1.08$ \\
\hline J170325.05-051742 & $\cdots$ & $0.021 \pm 0.0082$ & $0.199 \pm 0.018$ & $2.35 \pm 0.24$ & $11.66 \pm 1.42$ & -0.39 & $28.77 \pm 0.96$ & $1.02 \pm 0.27$ & -1.05 \\
\hline J170746.08-093916 & $\cdots$ & $0.119 \pm 0.0073$ & $0.342 \pm 0.020$ & $3.46 \pm 0.28$ & $3.27 \pm 1.26$ & -0.52 & $10.86 \pm 0.52$ & $<1.02$ & $<-0.51$ \\
\hline J193622.58-335420 & $\cdots$ & $0.031 \pm 0.0069$ & $0.127 \pm 0.016$ & $2.34 \pm 0.14$ & $5.27 \pm 0.96$ & 0.00 & $5.27 \pm 0.51$ & $1.86 \pm 0.36$ & -0.45 \\
\hline J195141.22-042024 & $\cdots$ & $0.030 \pm 0.0178$ & $0.065 \pm 0.036$ & $2.55 \pm 0.15$ & $8.56 \pm 1.02$ & -0.38 & $20.52 \pm 1.09$ & $<1.05$ & $<-0.91$ \\
\hline J195801.72-074609 & $\ldots$ & $0.056 \pm 0.0086$ & $0.203 \pm 0.018$ & $3.29 \pm 0.16$ & $7.44 \pm 1.06$ & -0.64 & $32.79 \pm 1.06$ & $<0.93$ & $<-0.90$ \\
\hline J200048.58-280251 & $\ldots$ & $0.027 \pm 0.0169$ & $0.113 \pm 0.017$ & $3.21 \pm 0.17$ & $7.19 \pm 1.20$ & -0.33 & $15.33 \pm 0.66$ & $<0.96$ & $<-0.87$ \\
\hline
\end{tabular}


Table 1

(Continued)

\begin{tabular}{|c|c|c|c|c|c|c|c|c|c|}
\hline WISE Name & $\begin{array}{c}R \text {-band } \\
\text { app. mag. } \\
\text { (Vega) }\end{array}$ & $\begin{array}{c}f_{3.4 \mu \mathrm{m}} \\
(\mathrm{mJy}) \\
W I S E\end{array}$ & $\begin{array}{c}f_{4.6 \mu \mathrm{m}} \\
(\mathrm{mJy}) \\
W I S E\end{array}$ & $\begin{array}{l}f_{12 \mu \mathrm{m}} \\
(\mathrm{mJy}) \\
W I S E\end{array}$ & $\begin{array}{l}f_{22 \mu \mathrm{m}} \\
(\mathrm{mJy}) \\
W I S E\end{array}$ & $\begin{array}{c}q_{22} \\
\text { Observed } \\
\text { frame }\end{array}$ & $\begin{array}{c}f_{1.4 \mathrm{GHz}} \\
(\mathrm{mJy}) \\
\text { NVSS }\end{array}$ & $\begin{array}{c}f_{870 \mu \mathrm{m}} \\
(\mathrm{mJy}) \\
\text { ALMA }\end{array}$ & $\begin{array}{c}\log \\
\left(f_{870 \mu \mathrm{m}} /\right. \\
\left.f_{22 \mu \mathrm{m}}\right)\end{array}$ \\
\hline J202148.06-261159 & $\ldots$ & $<0.015$ & $<0.065$ & $1.03 \pm 0.15$ & $6.27 \pm 1.01$ & -0.04 & $6.82 \pm 0.55$ & $4.4 \pm 0.38$ & -0.15 \\
\hline J204049.51-390400 & $\ldots$ & $0.070 \pm 0.0077$ & $0.254 \pm 0.017$ & $2.75 \pm 0.15$ & $4.02 \pm 0.91$ & -0.44 & $10.95 \pm 0.57$ & $5.1 \pm 0.43$ & 0.10 \\
\hline J205946.93-354134 & $\ldots$ & $0.052 \pm 0.0069$ & $0.182 \pm 0.015$ & $2.94 \pm 0.14$ & $4.75 \pm 0.99$ & -0.28 & $9.13 \pm 1.07$ & $<0.99$ & $<-0.40$ \\
\hline
\end{tabular}

Note.

${ }^{\mathrm{a}}$ SDSS $r$-band $\mathrm{AB}$ magnitude. 
Table 2

Herschel, JCMT, and CSO Photometry

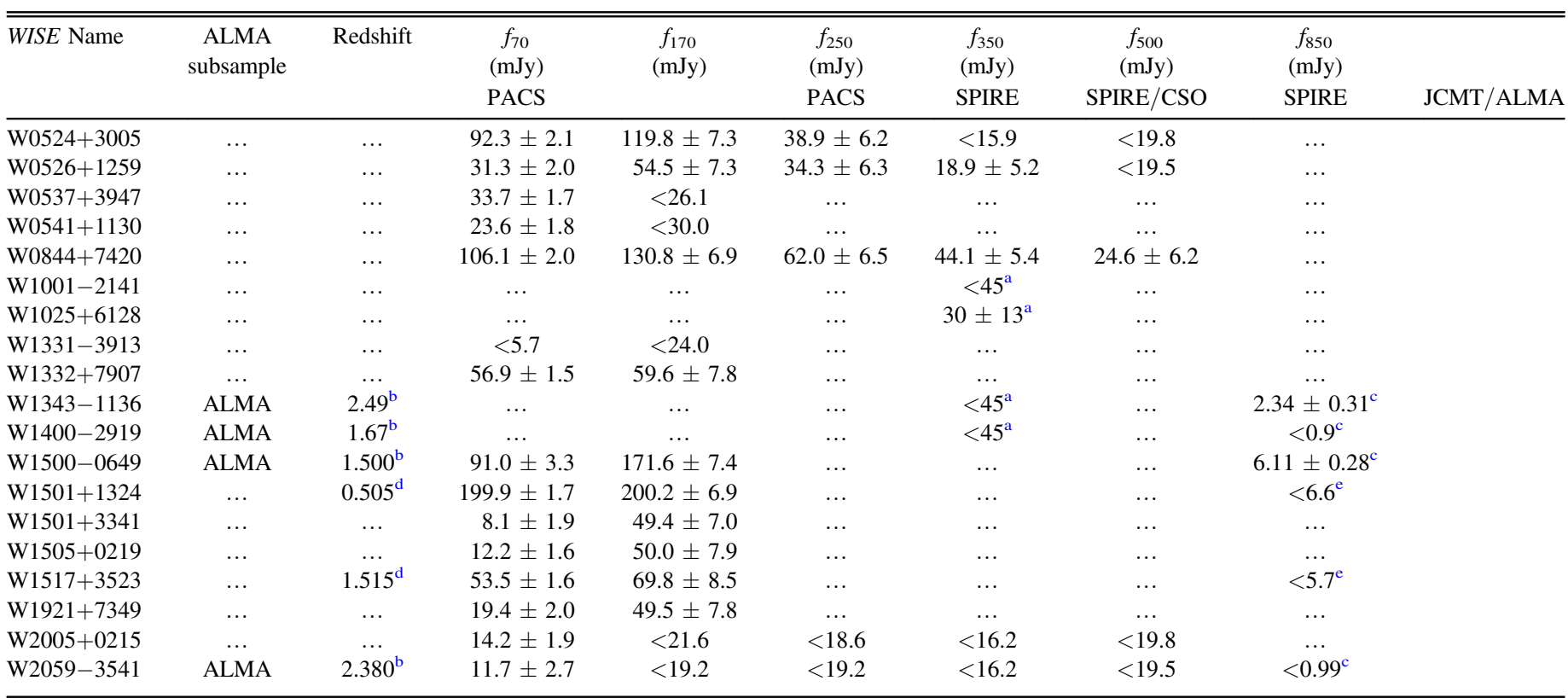

Notes.

${ }^{\mathrm{a}} \mathrm{CSO}$.

${ }^{\mathrm{b}}$ See Table 3 .

c ALMA $870 \mu$ m flux.

${ }^{\mathrm{d}}$ Palomar 200 inch.

e Jones et al. (2015).

the cryogen depletion of the Herschel Telescope in 2013 April. For the PACS observations, two concatenated mini-scans orientated at $70^{\circ}$ and $110^{\circ}$ were acquired. Each mini-scan has eight legs, a scan length of $3^{\prime}$, and a leg separation of $5^{\prime \prime}$, with a time on source of $220 \mathrm{~s}$. The SPIRE observations were conducted using the small jiggle map mode with two repeats, with $74 \mathrm{~s}$ time on source. The data were processed with HIPE 11.1.0. The aperture-corrected flux densities for all 15 Herschel-observed sources are presented in Table 2.

Summarizing, the FIR/submillimeter data are available for the 49 ALMA-observed sources discussed in this paper, PACS observations are available for two sources, W1500-0649 and W2059-3541, and SPIRE observations for one, W2059-3541. Two additional sources have upper limits from the CSO at $350 \mu \mathrm{m}, \mathrm{W} 1343-1136$ and W1400-2919.

\subsection{Optical and NIR Photometry and Spectroscopy}

Redshifts for 45 of the 49 ALMA sources and $R$-band photometry for 26 were obtained and are presented in Tables 1 and 3 . In total 48 of the 49 sources were observed spectroscopically, and three of these yielded no line detections. Full details of the spectroscopy will be presented in a later publication; here we make use only of the redshifts. Optical spectra of 23 objects were obtained using the Goodman spectrograph on the SOAR $4.2 \mathrm{~m}$ telescope on UT 2012 January 21-24 and UT 2012 December 9-12. The $R$-band photometry obtained with SOAR was taken with the spectrograph acquisition camera. We observed five sources from the WISE-NVSS-ALMA sample, and two from the northern JCMT sample, using the Double Spectrograph on the $5 \mathrm{~m}$ Hale telescope at Palomar Observatory between 2012 November and 2013 March. As described in more detail by
Kim et al. (2013), 24 sources were observed in the NIR with Magellan on UT 2012 July 27-29. Finally, 28 sources were observed with VLT/XShooter on UT 2013 June 4-6 and 31 sources were observed in $K_{s}$ with VLT/ISAAC over three nights from UT 2013 June 1-4, of which 14 were also observed in $J$.

\section{OBSERVATIONAL RESULTS}

\subsection{Photometry and Redshifts}

The $R$ and $r$ magnitudes and the WISE, NVSS, and ALMA flux densities for the 49 sources are presented in Table 1 . There are 26 ALMA detections at $3 \sigma$ or above. None of the sources are resolved. The two sources from the ALMA subsample observed with Herschel were both detected at $70 \mu \mathrm{m}$, and W1500-0649 also at $170 \mu \mathrm{m}$ (Table 2). Twelve additional sources from the full sample have Herschel detections. Neither of the two ALMA sources observed with the CSO, W1343-1136 and W1400-2919, was detected, resulting in $3 \sigma$ upper limits of $45 \mathrm{mJy}$ for each, while one of the other two souces, W1025+6128, has a modest detection, as listed in Table 2 . There are available $R$-band Vega system magnitudes from SOAR for 16 of the ALMA-detected sources and for 10 of the sources with upper limits. W0823-0624 and W1434-0235 have SDSS data in one or more bands. The $R / r$ magnitudes range from 20.7 to 24.1 . The optical photometry is used in this paper only as a rough constraint on the mass of the stellar populations; a full analysis of these data and the NIR photometry will be presented by A. Blain et al. (2015, in preparation).

Redshifts are available for 25 of the 26 detected sources, and for 20 of the 23 sources with ALMA upper limits. Six of the 
Table 3

Redshifts, Luminosities and Radio Power

\begin{tabular}{|c|c|c|c|c|c|c|c|c|c|c|c|c|c|}
\hline \multirow[t]{2}{*}{ WISE Name } & \multirow[t]{2}{*}{ Redshift } & \multicolumn{2}{|c|}{$\begin{array}{c}\log L_{\mathrm{AGN}} \\
\left(L_{\odot}\right)\end{array}$} & \multicolumn{4}{|c|}{$\begin{array}{c}\log L_{\mathrm{BB}} \\
\left(L_{\odot}\right)\end{array}$} & \multicolumn{3}{|c|}{$\begin{array}{c}\log L_{\text {Total }} \\
\left(L_{\odot}\right)\end{array}$} & \multirow{2}{*}{$\begin{array}{c}T_{\text {dust }} \\
(\mathrm{K})^{\mathrm{a}} \\
\text { Best model }\end{array}$} & \multirow[t]{2}{*}{$\begin{array}{l}\log P_{3 \mathrm{GHz}} \\
\left(\mathrm{W} \mathrm{Hz}^{-1}\right)\end{array}$} & \multirow[t]{2}{*}{$\begin{array}{c}q_{22} \\
\text { k-corrected }\end{array}$} \\
\hline & & Min & Max & $30 \mathrm{~K}$ & $50 \mathrm{~K}$ & $90 \mathrm{~K}$ & $120 \mathrm{~K}$ & Min & Max & Best & & & \\
\hline W0304-3108 & 1.54 & 13.01 & 13.16 & 11.49 & 12.46 & 12.92 & $\ldots$ & 13.02 & 13.36 & 13.36 & 90 & 26.58 & -1.30 \\
\hline W0306-3353 & 0.78 & 12.19 & 12.23 & 10.98 & 12.07 & 12.28 & $\ldots$ & 12.25 & 12.54 & 12.53 & $\langle 50,90\rangle$ & 24.92 & -0.55 \\
\hline W0354-3308 & 1.37 & 12.70 & 12.72 & $<11.14$ & $<12.14$ & $<12.59$ & $\ldots$ & $<12.71$ & $<12.96$ & $<12.96$ & 90 & 25.61 & -1.15 \\
\hline W0404-2436 & 1.26 & 12.58 & 12.63 & 11.46 & 12.48 & 12.93 & $\ldots$ & 12.61 & 13.11 & 13.11 & 90 & 25.67 & -1.69 \\
\hline W0409-1837 & 0.67 & 12.73 & 12.74 & $\ldots$ & $<11.99$ & $<12.4$ & $\ldots$ & $<12.80$ & $<12.90$ & $<12.90$ & 90 & 25.65 & -0.28 \\
\hline W0417-2816 & 0.94 & 11.95 & 12.01 & 11.48 & 12.55 & 12.98 & $\ldots$ & 12.12 & 13.02 & 12.47 & $\langle 30,50\rangle$ & 25.54 & -1.38 \\
\hline W0439-3159 & 2.82 & 13.44 & 13.45 & 12.04 & 12.84 & 13.33 & 14.17 & 13.47 & 14.24 & 13.70 & 90 & 26.76 & -1.12 \\
\hline W0519-0813 & $2.05^{\mathrm{b}}$ & 13.01 & 13.02 & $<11.30$ & $<12.2$ & $<12.68$ & $<13.58$ & $<13.03$ & $<13.68$ & $<13.18$ & 90 & 26.53 & -1.09 \\
\hline W0525-3614 & 1.69 & 12.51 & 12.60 & $<11.29$ & $<12.25$ & $<12.71$ & $<13.75$ & $<12.62$ & $<13.77$ & $<12.94$ & 90 & 25.64 & -0.55 \\
\hline W0526-3225 & 1.98 & 13.44 & 13.54 & 12.40 & 13.31 & 13.79 & $\ldots$ & 13.57 & 13.95 & 13.95 & 90 & 27.33 & -1.18 \\
\hline W0536-2703 & 1.79 & 13.05 & 13.05 & $\ldots$ & $\ldots$ & 12.94 & $\ldots$ & 13.00 & 13.30 & 13.30 & 90 & 25.90 & -0.54 \\
\hline W0549-3739 & 1.71 & 12.56 & 12.56 & 11.39 & 12.34 & 12.81 & $\ldots$ & 12.59 & 13.00 & 13.00 & 90 & 25.97 & -0.92 \\
\hline W0612-0622 & 0.47 & 12.03 & 12.04 & 10.89 & 12.05 & 12.20 & $\ldots$ & 12.07 & 12.43 & 12.43 & 90 & 25.14 & -0.32 \\
\hline W0613-3407 & 2.18 & 13.20 & 13.20 & $<11.43$ & $<12.31$ & $<12.79$ & $<13.68$ & $<13.21$ & $<13.80$ & $<13.34$ & 90 & 26.57 & -0.90 \\
\hline W0614-0936 & $\ldots{ }^{\mathrm{c}}$ & 12.94 & 12.95 & $<11.40$ & $<12.31$ & $<12.78$ & $<13.69$ & $<12.96$ & $<13.76$ & $<13.17$ & 90 & 25.87 & -0.38 \\
\hline W0630-2120 & 1.44 & 12.52 & 12.57 & 11.72 & 12.71 & 13.17 & $\ldots$ & 12.63 & 13.26 & 12.82 & $\langle 30,50\rangle$ & 25.86 & -1.33 \\
\hline W0642-2728 & 1.34 & 12.36 & 12.36 & 11.34 & 12.35 & $\ldots$ & $\ldots$ & 12.40 & 12.66 & 12.66 & 50 & 25.50 & -1.42 \\
\hline W0652-2006 & 0.60 & 11.58 & 11.59 & 11.11 & 12.24 & $\ldots$ & $\ldots$ & 11.71 & 12.33 & 12.12 & $\langle 30,50\rangle$ & 24.74 & -0.39 \\
\hline W0702-2808 & $0.94^{\mathrm{b}}$ & 12.04 & 12.04 & $<11.10$ & $<12.17$ & $\ldots$ & $\ldots$ & $<12.09$ & $<12.41$ & $<12.41$ & 50 & 25.23 & -1.00 \\
\hline W0714-3635 & 0.88 & 11.88 & 11.89 & 11.18 & 12.26 & & $\ldots$ & 11.97 & 12.41 & 12.41 & 50 & 25.34 & -1.09 \\
\hline W0719-3349 & 1.63 & 12.57 & 12.57 & $\ldots$ & 12.75 & 13.22 & $\cdots$ & 12.97 & 13.31 & 13.01 & $\langle 50,90\rangle$ & 26.71 & -1.33 \\
\hline W0811-2225 & 1.11 & 12.63 & 12.63 & $\ldots$ & $\ldots$ & $<12.65$ & $\ldots$ & $<12.94$ & $<12.94$ & $<12.94$ & 90 & 25.75 & -1.53 \\
\hline W0823-0624 & 1.75 & 13.11 & 13.22 & $<11.36$ & $<12.3$ & $<12.76$ & $\ldots$ & $<13.12$ & $<13.35$ & $<13.35$ & 90 & 26.70 & -1.10 \\
\hline W1308-3447 & 1.65 & 12.99 & 12.99 & 11.22 & 12.18 & 12.65 & $\ldots$ & 13.00 & 13.15 & 13.15 & 90 & 26.74 & -1.40 \\
\hline W1343-1136 & 2.49 & 13.00 & 13.02 & $\ldots$ & 12.43 & 12.92 & 13.78 & 13.12 & 13.85 & 13.27 & 90 & 26.23 & -0.75 \\
\hline W1400-2919 & 1.67 & 13.10 & 13.11 & $<10.78$ & $<11.73$ & $\ldots$ & $<13.16$ & $<13.11$ & $<13.43$ & $<13.43$ & 120 & 26.63 & -1.13 \\
\hline W1412-2020 & 1.82 & 13.02 & 13.17 & 11.52 & 12.45 & 12.92 & $\ldots$ & 13.03 & 13.36 & 13.36 & 90 & 25.95 & -0.43 \\
\hline W1434-0235 & 1.92 & 12.89 & 12.89 & $<11.08$ & $<12.00$ & $<12.47$ & $<13.39$ & $<12.90$ & $<13.51$ & $<13.03$ & 90 & 26.62 & -1.22 \\
\hline W1439-3725 & 1.19 & 12.29 & 12.29 & $<10.79$ & $<11.81$ & $<12.26$ & $\ldots$ & $<12.30$ & $<12.58$ & $<12.58$ & 90 & 25.58 & -1.70 \\
\hline W1500-0649 & 1.50 & 13.07 & 13.50 & $\ldots$ & 12.84 & 13.33 & $\ldots$ & 13.52 & 13.59 & 13.52 & 90 & 26.10 & -0.89 \\
\hline W1510-2203 & 0.95 & 12.60 & 12.61 & $<10.74$ & $<11.81$ & $<12.23$ & $\ldots$ & $<12.62$ & $<12.76$ & $<12.76$ & 90 & 25.57 & -0.84 \\
\hline W1513-2210 & 2.20 & 13.26 & 13.26 & 11.86 & 12.74 & 13.22 & $\ldots$ & 13.28 & 13.54 & 13.54 & 90 & 26.68 & -0.89 \\
\hline W1514-3411 & 1.09 & 12.44 & 12.48 & $<10.81$ & $<11.86$ & $<12.29$ & $\ldots$ & $<12.45$ & $<12.70$ & $<12.70$ & 90 & 25.57 & -1.36 \\
\hline $\mathrm{W} 1521+0017$ & $2.63^{\mathrm{b}}$ & 13.61 & 13.61 & $\ldots$ & $\ldots$ & 12.63 & $\ldots$ & 13.65 & 13.65 & 13.65 & 90 & 25.60 & -1.04 \\
\hline W1541-1144 & 1.58 & 12.81 & 12.94 & 11.13 & 12.10 & 12.57 & $\ldots$ & 12.95 & 13.01 & 13.01 & 90 & 26.40 & -1.15 \\
\hline W1634-1721 & 2.08 & 12.83 & 12.83 & $<11.08$ & $<11.98$ & $<12.46$ & $<13.36$ & $<12.84$ & $<13.47$ & $<12.98$ & 90 & 26.11 & -0.80 \\
\hline W1641-0548 & 1.84 & 12.94 & 13.09 & & 12.41 & 12.88 & $\ldots$ & 13.05 & 13.30 & 13.30 & 90 & 25.83 & -0.37 \\
\hline W1653-0102 & 2.02 & 13.00 & 13.00 & $<11.04$ & $<11.94$ & $<12.41$ & $<13.31$ & $<13.00$ & $<13.48$ & $<13.10$ & 90 & 26.19 & -0.73 \\
\hline W1657-1740 & $\ldots^{\mathrm{c}}$ & 13.16 & 13.17 & $<11.03$ & $<11.94$ & $<12.42$ & $<13.32$ & $<13.17$ & $<13.55$ & $<13.60$ & $\langle 90,120\rangle$ & 26.34 & -0.68 \\
\hline W1702-0811 & 2.85 & 13.60 & 13.60 & $<11.28$ & $<12.07$ & $-\ldots$ & $<13.4$ & $<13.60$ & $<13.81$ & $<13.81$ & 120 & 27.26 & -1.20 \\
\hline W1703-0517 & $1.80^{\mathrm{b}}$ & 13.11 & 13.51 & 11.12 & 12.05 & 12.48 & 13.45 & 13.12 & 13.61 & 3.60 & $\langle 90,120\rangle$ & 26.91 & -0.74 \\
\hline W1707-0939 & $\ldots{ }^{c}$ & 12.98 & 13.04 & $<11.15$ & $<12.06$ & $<12.54$ & $<13.43$ & $<12.99$ & $<13.56$ & $<13.56$ & 120 & 26.14 & -0.89 \\
\hline W1936-3354 & $2.24^{\mathrm{b}}$ & 13.05 & 13.18 & 11.45 & 12.32 & $\ldots$ & 13.69 & 13.06 & 13.78 & 13.78 & 120 & 25.64 & -0.40 \\
\hline W1951-0420 & 1.58 & 12.80 & 12.83 & $<11.08$ & $<12.05$ & $\ldots$ & $<13.45$ & $<12.84$ & $<13.54$ & $<13.54$ & 120 & 26.18 & -1.03 \\
\hline W1958-0746 & 1.80 & 12.98 & 12.98 & $<11.08$ & $<12.01$ & $<12.48$ & $<13.41$ & $<12.99$ & $<13.54$ & $<13.10$ & 90 & 26.51 & -0.99 \\
\hline W2000-2802 & 2.28 & 12.75 & 12.90 & $<10.72$ & $<11.59$ & $<12.07$ & $<12.96$ & $<12.75$ & $<13.17$ & $<12.96$ & 90 & 25.99 & -0.73 \\
\hline
\end{tabular}


Table 3

(Continued)

\begin{tabular}{|c|c|c|c|c|c|c|c|c|c|c|c|c|c|}
\hline \multirow[t]{2}{*}{ WISE Name } & \multirow[t]{2}{*}{ Redshift } & \multicolumn{2}{|c|}{$\begin{array}{c}\log L_{\mathrm{AGN}} \\
\left(L_{\odot}\right)\end{array}$} & \multicolumn{4}{|c|}{$\begin{array}{c}\log L_{\mathrm{BB}} \\
\left(L_{\odot}\right)\end{array}$} & \multicolumn{3}{|c|}{$\begin{array}{c}\log L_{\text {Total }} \\
\left(L_{\odot}\right)\end{array}$} & \multirow{2}{*}{$\begin{array}{c}T_{\text {dust }} \\
(\mathrm{K})^{\mathrm{a}} \\
\text { Best model }\end{array}$} & \multirow[t]{2}{*}{$\begin{array}{l}\log P_{3 \mathrm{GHz}} \\
\left(\mathrm{W} \mathrm{Hz}^{-1}\right)\end{array}$} & \multirow[t]{2}{*}{$\begin{array}{c}q_{22} \\
\text { k-corrected }\end{array}$} \\
\hline & & Min & Max & $30 \mathrm{~K}$ & $50 \mathrm{~K}$ & $90 \mathrm{~K}$ & $120 \mathrm{~K}$ & Min & $\operatorname{Max}$ & Best & & & \\
\hline W2021-2611 & 2.44 & 12.97 & 13.51 & 11.85 & 12.68 & $\ldots$ & 14.04 & 13.00 & 14.15 & 14.15 & 120 & 25.93 & -0.45 \\
\hline W2040-3904 & $\ldots^{c}$ & 12.92 & 13.15 & 11.85 & 12.76 & 13.23 & & 12.96 & 13.49 & 13.49 & 90 & 26.14 & -0.81 \\
\hline W2059-3541 & 2.38 & 13.15 & 13.37 & $\ldots$ & $\ldots$ & $<12.46$ & $<12.87$ & $<13.15$ & $<13.42$ & $<13.42$ & 90 & 26.26 & -0.69 \\
\hline
\end{tabular}

Notes.

${ }^{\mathrm{a}}\langle\mathrm{T} 1, \mathrm{~T} 2\rangle$ denotes an average between these two model fits.

${ }^{\mathrm{b}}$ Uncertain redshift: single line, blended lines, or weak lines.

${ }^{\mathrm{c}} z=2$ assumed if no spectroscopic redshift exists. 


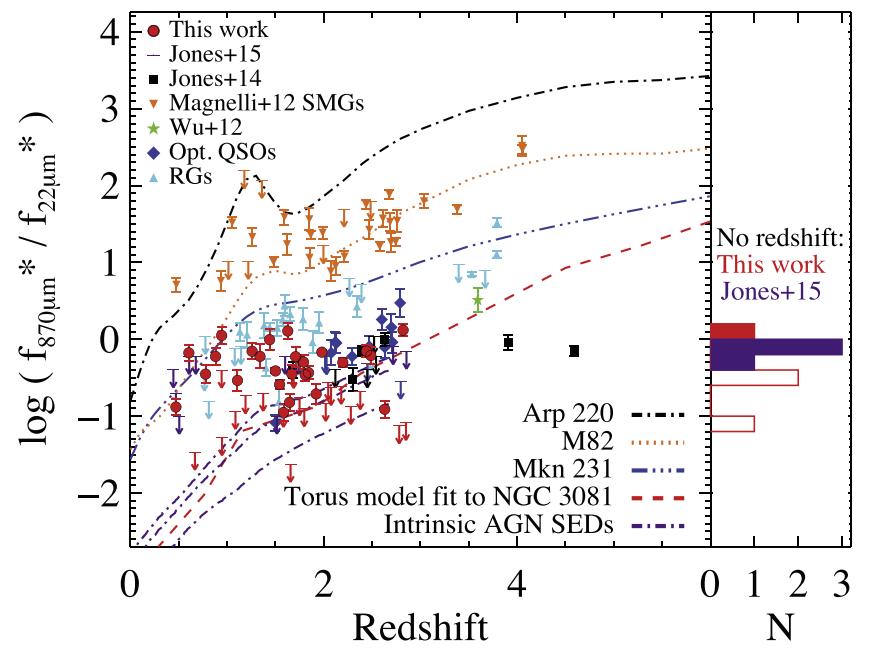

Figure 3. Ratio of ALMA $870 \mu \mathrm{m}$ to WISE $22 \mu \mathrm{m}$ flux density vs. redshift for the ALMA sample (red points and limits). Histogram: sources without redshift (submillimeter-detected sources: filled bars; upper limits: empty bars). The comparison templates and source samples are described in the text. The three intrinsic AGN curves (purple dashed-dotted lnes) are the mean $\left[\log \left(L_{2-10 \mathrm{kev}} /\right.\right.$ $\left.\left.\operatorname{erg~s}^{-1}\right)<42.9\right]$, mean[all], mean $\left[\log \left(L_{2-10 \mathrm{kev}} / \mathrm{erg} \mathrm{s}^{-1}\right)>42.9\right]$ SEDs of Mullaney et al. (2011) in descending order which cover the 6-1000 $\mu \mathrm{m}$ rest frame; the full dispersion of intrinsic AGN SEDs reported in that paper is significantly wider. The three ALMA sources lying on or above the Mkn 231 template are W0417-2816, W0652-2006, and W0714-3635. *: The comparison samples include data at $850 \mu \mathrm{m}$ and at $24 \mu \mathrm{m}$.

nineteen sources in Table 2 have known redshifts, including the four sources in the ALMA subsample and the two JCMTobserved sources. It is beyond the scope of the present paper to analyze the optical/NIR spectroscopy, but we note that many spectra have indications from the ionization levels of an obscured radiative-mode AGN (Baldwin et al. 1981; Kewley et al. 2013). The Magellan/FIRE observations have been published by Kim et al. (2013) while the VLT/Xshooter observations will be reported by A. Blain et al. (2015, in preparation). One object was not observed (W1657-0102), one was not detected even in continuum (W0614-0936), and for two objects only a faint continuum was detected (W1707-0811 and W2040-3541). Additionally the redshifts are uncertain for five sources that have only weak lines, a single line detection, or an unresolved line pair. They are: W0519-0813 (L $\alpha$ $\lambda 1216 \AA$ ), W0702-2808 ([O II $] \lambda 3727 \AA$ ), W1521+0017 (weak $\mathrm{H} \beta$, [O III] $\lambda 4959,5007 \AA$ ), W1703-0517 (blended H $\alpha+\left[\mathrm{N}_{\text {II }}\right]$ $\lambda 6584 \AA$ ), and W1936-3354 ([O II] $\lambda 3727 \AA$ ).

\subsection{Colors and SEDs}

The ALMA observations were designed to constrain the luminosity of these quasars in the rest-frame FIR-submillimeter compared to the MIR. We will show that most of our WISE-NVSS-ALMA sample have SEDs dominated by an AGN in the MIR, and possibly through the FIR-submillimeter also. However, substantial rates of star formation are likely also present.

We present the main results in Figures 3-5. We show these results in two complementary ways: (1) the ratio of $870 \mu \mathrm{m} /$ $22 \mu \mathrm{m}$ flux density as a function of redshift is shown in Figure 3, and (2) we use the range in redshifts for the sample to construct an "ensemble" rest-frame SED for our sample in Figure 4. We show ensemble SEDs of several comparison samples in Figure 5. In all the plots we also show the tracks for
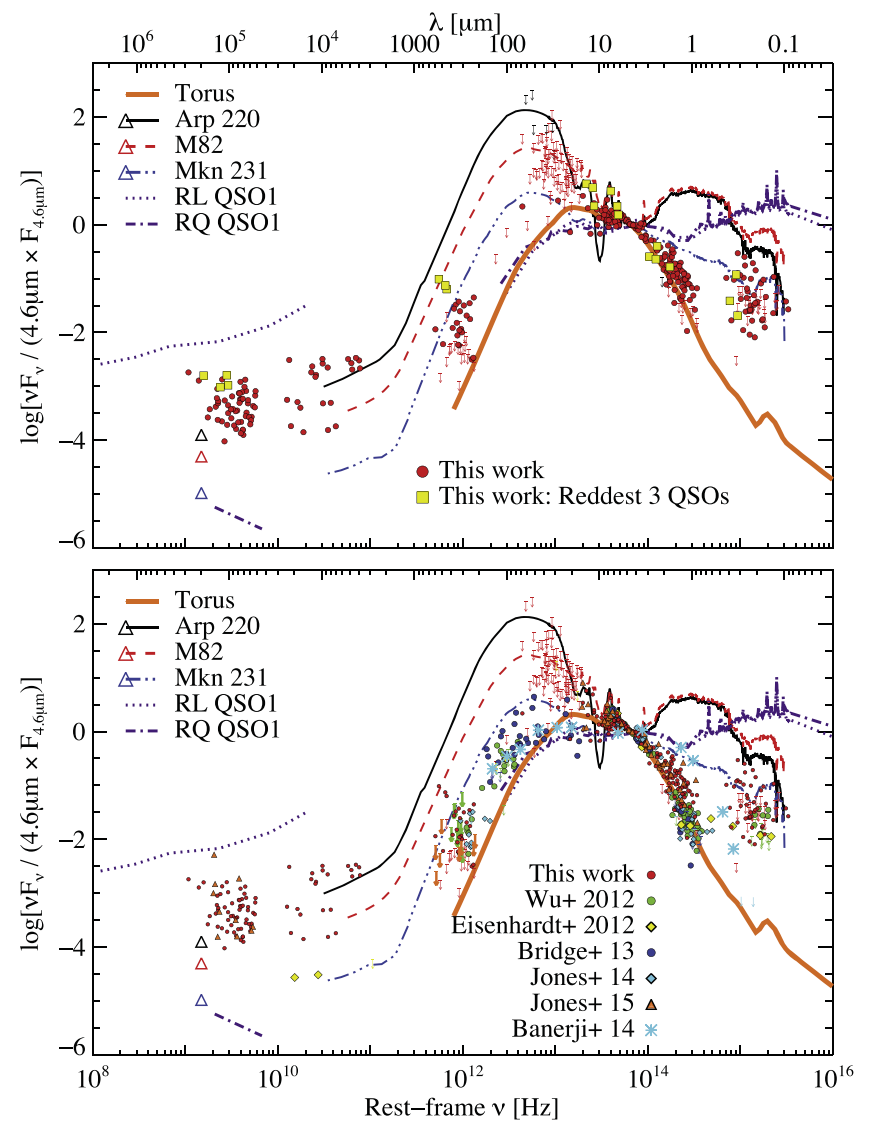

Figure 4. Rest-frame ensemble SEDs for WISE-selected ultra-red systems. Top: the 45 sources in our ALMA sample with known redshift, normalized at $4.6 \mu \mathrm{m}$, compared with the templates from the library of Polletta et al. (2007), the torus model fitted to one of our sources in Section 5.2.1, and the intrinsic AGN SEDs of Shang et al. (2011) (labelled RQ QSO1 and RL QSO1). The colored triangles in the radio correspond to the matched color template. The three reddest objects, relative to Mkn 231, from Figure 3 are highlighted in yellow. The upper limits in the rest $\sim 12-100 \mu \mathrm{m}$ range are largely from IRAS, with a few from Herschel (see text for details). Several sources lie at redshifts where the $22 \mu \mathrm{m}$ band coincides with the $9.7 \mu \mathrm{m}$ silicate absorption feature, which may partially account for the turnover in their spectral shapes. Note the intermediate radio power compared to classical (evolved) RQ and RL QSOs, when normalized to $4.6 \mu \mathrm{m}$ power. Bottom: Rest-frame SEDs for the WISEselected red Hot DOG sources from Wu et al. (2012) (green circles), Eisenhardt et al. (2012) (yellow diamonds), Jones et al. (2014) (cyan diamonds), and the WLABs from Bridge et al. (2013) (blue circles) compared to our samples (red circles, orange triangles; Jones et al. 2015). Also shown are the reddest of the dust-reddened type 1 quasars from Banerji et al. (2014) (cyan asterisks).

several templates of nearby well studied sources. The ensemble SEDs must be interpreted carefully because the choice of normalization wavelength affects the relative appearance of dispersion between the points at other wavelengths. We have chosen to normalize the templates and data at rest-frame $4.6 \mu \mathrm{m}$. The selection of $4.6 \mu \mathrm{m}$ has the disadvantage that this spectral region may suffer significant extinction from a thick torus or other nuclear dusty structure, but it is the longest wavelength (and hence has the lowest optical depth) that avoids the PAH and silicate features yet still lies within the WISE restframe wavelength range for all of the ALMA sources.

The source templates in all five figures are from Polletta et al. (2007), and include the cool starburst-dominated ultraluminous infrared galaxies (ULIRG) Arp 220, the starburst M82, and the broad-line dusty QSO Mrk 231. The torus model in Figures 4 and 5 is based on the tapered disk models of 

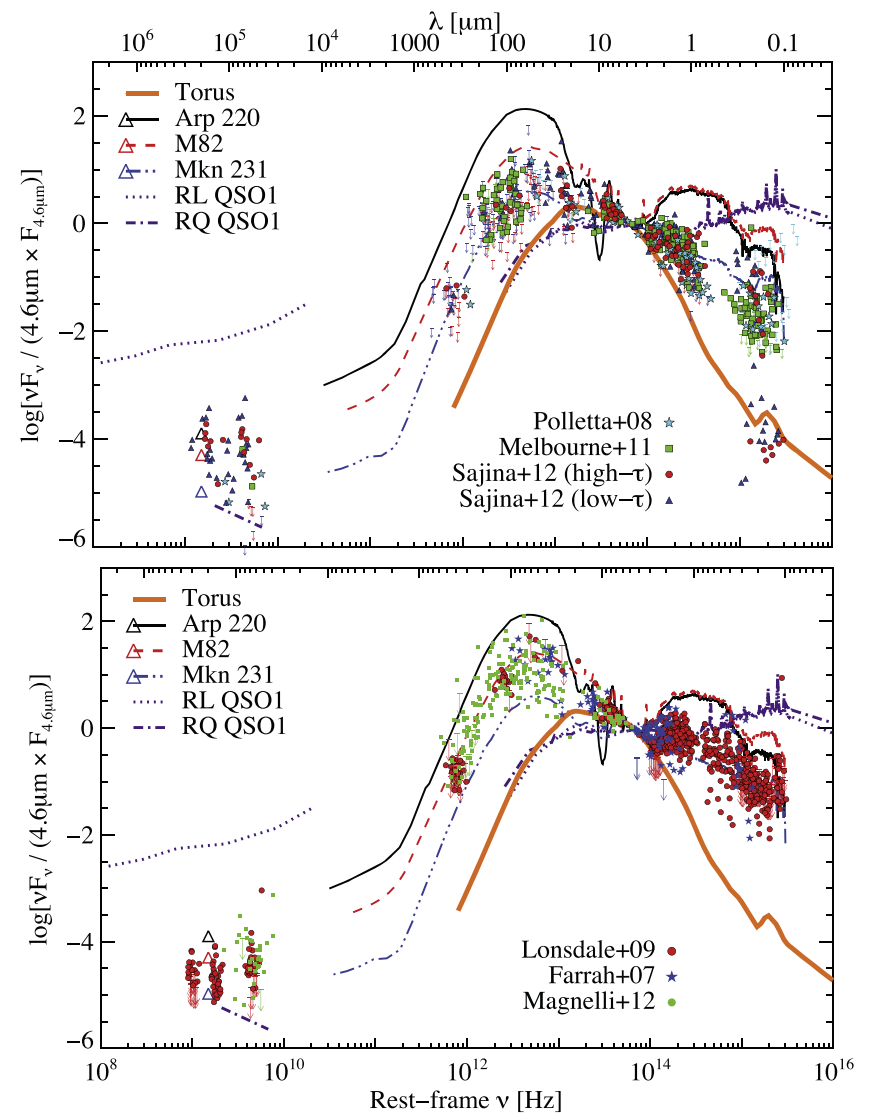

Figure 5. Rest-frame SEDs for several comparison Spitzer and Herschel samples. Top: rest-frame SEDs for 152 Spitzer DOGs which were selected in a similar manner to our sample (Polletta et al. 2008; Melbourne et al. 2012; Sajina et al. 2012). The extreme redness in the UV-optical for many of the Sajina et al. (2012) sources is due to their selection criteria. Bottom: rest-frame SEDs for 61 high-redshift MIR-selected starburst-dominated ULIRGs from Lonsdale et al. (2009) and Fiolet et al. (2009) (red symbols), 16 Spitzer $70 \mu \mathrm{m}$ selected galaxies from Farrah et al. (2007) (blue symbols) and 61 SMGs with Herschel data from Magnelli et al. (2012) (green symbols).

Efstathiou \& Rowan-Robinson (1995) and has been fitted to one of our best sampled SEDs in this paper (see Section 5.2.1). This torus model has an opening angle of $45^{\circ}$, an inclination angle of $54^{\circ}$ and a UV equatorial optical depth $\tau_{\nu}=500$. The intrinsic AGN SEDs, from Shang et al. (2011) and Mullaney et al. (2011), are empirical SEDs of nearby AGNs from which the host galaxy light has been subtracted, and they are quite similar to each other and to other published intrinsic AGN SEDs (Elvis et al. 1994; Richards et al. 2006; Netzer et al. 2007; Assef et al. 2010). In Figure 3 we include the modeled SED of a dust torus of $\sim 4 \mathrm{pc}$ radius in the nearby AGN NGC 3081 that Ramos-Almeida et al. (2013) derived based on Herschel data and subarcsecond MIR imaging with Gemini T-ReCS.

Most of our WISE-NVSS-ALMA sample is much more strongly MIR-dominated than the starburst templates (M82 and Arp 220) in Figure 3, and also compared to the $1<z<3$ SMGs (Magnelli et al. 2012), which we illustrate to provide a comparison to high-redshift starburst-dominated systems. The WISE radio-blind Hot DOG samples (EWB12; Jones et al. 2014) and our northern sample of RP sources (Jones et al. 2015) are similar to the ALMA sample in showing a low submillimeter/MIR flux density ratio compared to the galaxy templates. Generally speaking, the WISE sources lie between the intrinsic SEDs and the Mkn 231 template, though some of the upper limits lie below even the intrinsic SEDs shown. We also show some representative redshift $\sim 1-3$ RGs (Archibald et al. 2001; Grimes et al. 2005; Seymour et al. 2007) and broadline (optically selected) quasars (Priddey et al. 2003, 2007) with available $850 \mu \mathrm{m}$ data in the plot, but a detailed comparison to the FIR-submillimeter properties of well selected samples of AGNs of various types is beyond the scope of this paper. The broad-line QSOs shown tend to have similar colors to our sample, while the RGs shown tend to be a bit more FIR-strong relative to the MIR, but these trends may be dominated by selection effects and should be used only as a very general comparison to our sample.

The conclusions from Figure 3 are emphasized in the ensemble SED (Figure 4-top), where we see a strong similarity among the MIR-submillimeter SEDs of the ALMA sample. They resemble the torus model with high optical depth in the rest-frame NIR-MIR, as expected since this model was designed to fit one of our sources, but they are systematically steeper than any of the galaxy or intrinsic AGN templates in this wavelength range, when normalized at $4.6 \mu \mathrm{m}$. There is a lack of observational data available in the rest-frame FIR, although the IRAS limits (red limits at $10<\lambda_{\text {rest }}<100 \mu \mathrm{m}$ ) help to constrain the flux of many of our WISE-NVSS sample, ruling out SEDs like Arp 220 and M82.

The three sources with highest submillimeter/MIR flux ratios (relative to the templates) from Figure 3, W0417-2816, W0652-2006, and W0714-3635 (yellow points in Figure 4), are the strongest candidates for possessing significant ongoing star formation. Their presence in our sample may be the result of a selection effect due to strong 6.7-7.7 $\mu \mathrm{m}$ PAH features falling in the $W 3$ filter, or an $11.7 \mu \mathrm{m}$ PAH feature falling in the $W 4$ filter, as can be seen in Figure 4.

In Figure 4-bottom we add the ten sources with known redshift from Jones et al. (2015) (orange triangles), which are drawn from our northern WISE-NVSS sample. They all have upper limits at $850 \mu \mathrm{m}$ from JCMT and they show very similar SEDs to the ALMA subsample. We also compare our radioselected samples to the radio-blind WISE Hot DOG samples of EWB12 and of Jones et al. (2014). We see a very close similarity between the radio-selected (red and orange symbols) and radio-blind samples (green, blue, cyan, and yellow filled symbols). The radio-blind Hot DOG samples have systematically larger redshifts than our sample, displacing the two sets of SEDs from each other in rest wavelength somewhat, but the radio-selected and radio-blind samples fall within a continuous band. The different redshift selection function for the two samples may be unrelated to radio power, instead being due at least in part to the redder $(W 2-W 3)$ threshold of EWB12. This probably eliminates sources with silicate absorption that falls into the $22 \mu \mathrm{m}$ filter, and therefore favors sources with $z \gtrsim$ 1.5-2. Jones et al. (2015) have suggested that the northern sources from our NVSS-WISE sample that they observed at $850 \mu \mathrm{m}$ at the JCMT may show slightly less steep rest-frame SEDs than the EWB12 samples. This is an interesting possibility that requires further study.

The WISE samples of EWB12 have more data from Herschel in the rest MIR-FIR than we do for our sample, and their ensemble FIR SEDs tend to fall in the region occupied by the intrinsic SEDs, the torus template, and the Mkn 231 template (Figure 4). The two WISE-NVSS sources for which we have Herschel data fall in the same region. All of these sources lie 
well below both M82 and Arp 220 in this wavelength region, emphasizing the result from Figure 3. The limited Herschel data that we do have for our sample, and the IRAS limits, support a picture in which our WISE-NVSS sample has similar FIR-submillimeter SEDs to the radio-blind WISE samples. The one Hot DOG with published radio data (Eisenhardt et al. 2012) has lower radio power than our sample, when normalized to $4.6 \mu \mathrm{m}$ power.

It is interesting to compare the WISE-NVSS objects to the reddest known broad-line type 1 quasar from Banerji et al. (2014), ULAS J1234+0907 $(z=2.50)$, shown as the cyan asterisks in Figure 4-bottom. The SED shape is very similar to the Hot DOGs through the MIR-submillimeter but is much less red than any of the WISE sample in the rest NIR $1-5 \mu \mathrm{m}$ region. Unlike the WISE red sample, ULAS J1234+0907 follows the Mkn 231 template through $\sim 1 \mu \mathrm{m}$, only dropping steeply at shorter wavelengths. The best fit model by Banerji et al. (2014) has $A_{V}=6$. This result emphasizes the likelihood that the steep red WISE-optical SEDs for the WISE-selected samples are caused by heavy obscuration.

In Figure 5-top we turn to a comparison to the Spitzerselected "power-law" DOGs (Polletta et al. 2008; Melbourne et al. 2012; Sajina et al. 2012), including the two $z>3$ Compton-thick quasars discussed by Polletta et al. (2008). As a class the WISE samples show significantly redder ensemble rest NIR slopes than most of the Spitzer DOG samples. The WISE sources also appear to turn over into the FIR/submillimeter at systematically shorter wavelength than the DOGs, as can be seen by comparing Figures 4-bottom and 5-top, and as previously discussed by EWB12 and Jones et al. (2014, 2015). To first order, this is likely to indicate a larger ratio of star formation to AGN accretion in the Spitzer DOGs than in the Hot DOGs.

Lastly we illustrate in Figure 5-bottom the ensemble SEDs of several samples of Spitzer-selected starburst-dominated ULIRGs (Farrah et al. 2007; Fiolet et al. 2009; Lonsdale et al. 2009) and SMGs observed with Herschel (Magnelli et al. 2012). These samples have more marked FIR emission than either the WISE sources or the Spitzer-selected DOGs, relative to MIR emission, and bluer optical-MIR SEDs.

Turning to the centimeter-radio emission, there is a steady decrease in the average radio $/ 4.6 \mu \mathrm{m}$ flux density ratio from our sample through the Spitzer DOGs to the SMGs/starbursts, although there is a lot of overlap between the latter two samples. This in entirely consistent with the selection in favor of bright radio sources in our sample, and the dominance of star formation in the SMGs and starbursts.

In summary, the WISE-selected ultra-red samples have very similar SED shapes from the rest-frame NIR through the FIR, with no obvious difference between the radio-selected samples (this paper and Jones et al. 2015) and the radio-blind Hot DOGs (EWB12; Jones et al. 2014) except that the radio-blind samples have a larger mean redshift, which may be caused by the different selection functions. Together, these sources are redder than any other known source type in the NIR-MIR, and most of them turn over into the FIR at higher frequencies than the Spitzer DOGs, starbursts, and SMGs.

\subsection{Synchrotron Contribution to the ALMA fluxes}

Before addressing the possible range of SFRs in these sources, we first consider the possibility that a fraction of the $345 \mathrm{GHz}$ flux is due to synchrotron emission. Since we have selected compact radio sources in radiatively efficient AGNs there is the possibility that the radio emission is beamed and that some of our sources are blazars. We briefly address the possibility that some of the $345 \mathrm{GHz}$ emission arises from nonthermal synchrotron emission associated with the radio sources here, but we defer detailed discussion of this topic to the next paper in our series, in which we present the high-resolution $X$ band $(8-12 \mathrm{GHz})$ imaging from the VLA.

We can draw some preliminary conclusions from the measured spectral indices across the 8-12 GHz VLA $X$-band, which are more reliable than indices derived from noncontemporaneous and non-beam-matched $1.4 \mathrm{GHz}$ NVSS data and the much later $X$-band imaging. We find that the majority of the sample has steep spectral indices between 8 and $12 \mathrm{GHz}$; 42 of them have $\alpha_{12}^{8}<-0.8$ (27 have indices steeper than -1.0 ), characteristic of optically thin synchrotron emission and potentially consistent with being Gigahertz Peaked Sources ( $<1 \mathrm{kpc}$ in size with a synchrotron peak $\sim 1 \mathrm{GHz}$ ) or Compact Steep Spectrum sources (CSS; $<20 \mathrm{kpc}$ in size with a synchrotron peak below $1 \mathrm{GHz}$ ) (O'Dea 1998). Several sources are also resolved or multiple on scales of $1-10 \mathrm{kpc}$. For most of these steep spectrum sources the synchrotron contribution to the $345 \mathrm{GHz}$ flux is likely to be $<10 \%$.

The remaining seven sources have flat or inverted $8-12 \mathrm{GHz}$ spectral indices, but two of these show a steepening spectral index between 12 and $20 \mathrm{GHz}$ (from our limited VLA $K$-band imaging), thus they are also likely to be dominated by optically thin synchrotron emission (W0526-3225 and W0823-0642). Two of the remaining five sources (W0642-2728 and W1434-0235) have an ALMA measurement (a detection and a limit, respectively) that is well in excess (by a factor of five or more) of the extrapolated flat radio SED. The other three sources (W0536-2703, W1412-2020, and W1634-1721) require a synchrotron peak beyond $12 \mathrm{GHz}$ to avoid exceeding the ALMA flux density detection or limit, and these are also probably not blazars unless they are exceptionally variable. They are more likely to be High Frequency Peakers (small $<100 \mathrm{pc}$ sources with synchrotron peaks above $4 \mathrm{GHz}$; Dallacasa et al. 2000) and it is possible that their $345 \mathrm{GHz}$ flux has a significant contribution from optically thin synchrotron emission. None of the flat or inverted spectrum sources has a plausible SED that can explain the WISE data as synchrotron emission from a blazar.

\section{ISM MASS, SED FITS, AND DERIVED PARAMETERS}

As noted in the previous section, the simplest description of the NIR-FIR SED shapes of the majority of our sample is that they resemble the intrinsic shapes of local AGN samples, derived by subtracting the host galaxy emission (Elvis et al. 1994; Richards et al. 2006; Netzer et al. 2007; Assef et al. 2010; Mullaney et al. 2011; Shang et al. 2011). If we interpret the SEDs in this fashion then we could conclude that all of the MIR-submillimeter dust emission stems from reradiation of accretion disk energy by a nuclear torus or other dusty structure. In this section we explore, as an alternative, the range of plausible contributions from star formation that may be permitted by reasonable models of the SEDs.

\subsection{Interstellar Medium Mass}

We derive the ISM masses assuming that $100 \%$ of the $345 \mathrm{GHz}$ flux is thermal dust emission. As noted in the 
previous section the thermal fluxes could be overestimated by $<10 \%$ for most of the sample due to possible contributions from synchrotron emission, and by up to $100 \%$ for three sources. We do not correct the data for this contamination because it is highly uncertain at this point in time.

Scoville et al. (2014) derive the gaseous ISM mass $\left(M_{\mathrm{HI}}+M_{\mathrm{H}_{2}}\right)$ of populations of distant galaxies in the COSMOS field using ALMA $870 \mu \mathrm{m}$ data as a measure of cool dust mass. They use a local sample of well studied galaxies to show that the observed ratio of $850 \mu \mathrm{m}$ specific luminosity to ISM mass is $L_{\nu 850 \mu \mathrm{m}} / M_{\mathrm{ISM}}=1 \pm 0.23 \times 10^{20}$ $\mathrm{erg} \mathrm{s}^{-1} \mathrm{~Hz}^{-1} M_{\odot}^{-1}$ for low-redshift spirals, with a dispersion of a factor of about 5. Using this empirical calibration, Scoville et al. (2014) derive the following relation for a flux density measured at observed frequency $\nu_{\text {obs }}$ (valid for $\lambda_{\text {rest }}>250 \mu \mathrm{m}$ on the Rayleigh-Jeans tail where the emission will be optically thin) to derive ISM masses from ALMA data for SMGs (their Equation (12), valid for a dust temperature of $25 \mathrm{~K}$ ):

$$
\frac{f_{\nu_{\mathrm{obs}}}}{\mathrm{mJy}}=0.83 \frac{M_{\mathrm{ISM}}}{10^{10} M_{\odot}}(1+z)^{4.8}\left(\frac{\nu_{\mathrm{obs}}}{\nu_{850 \mu \mathrm{m}}}\right)^{3.8} \frac{\Gamma_{\mathrm{RJ}}}{\Gamma_{0}}\left(\frac{\mathrm{Gpc}}{d_{\mathrm{L}}}\right)^{2} .
$$

Here $\Gamma_{\mathrm{RJ}} / \Gamma_{0}$ corrects for the departure of the dust emission spectrum from the Rayleigh-Jeans tail as the redshift increases from 0 (and the rest frequency approaches the peak of the spectrum) and $d_{\mathrm{L}}$ is the luminosity distance. Scoville et al. (2014) adopt $250 \mu \mathrm{m}$ as the minimum acceptable rest-frame wavelength for derivations of ISM mass, thus we expect our results to provide reasonable estimates for sources with $z<2.5$, which represents $94 \%$ of our sample, with only small inaccuracies for the three sources with $2.5<z<2.85$. $\Gamma_{\mathrm{RJ}}$ is a function of assumed dust temperature, which was taken to be $25 \mathrm{~K}$ by Scoville et al. (2014). The constant 0.83 in the equation is also proportional to $T_{\mathrm{d}}$. We derive ISM masses (Table 5, columns 13 and 14), for dust temperatures of 30 and $90 \mathrm{~K}$, using the appropriate values for the constant, $\Gamma_{0}$, and $\Gamma_{\mathrm{RJ}}$. The majority of the dust in these systems is unlikely to be as warm as $90 \mathrm{~K}$, therefore these values provide fairly strong lower limits to the ISM masses when we have an $870 \mu \mathrm{m}$ detection. We estimate the overall uncertainty to be a factor of five, based on the unknown dust temperature and the overall dispersion for local starburst systems in Figure 1 of Scoville et al. (2014).

The ISM masses for $T_{\mathrm{d}}=30 \mathrm{~K}$ range from 0.8 to $56 \times 10^{10}$ $M_{\odot}$, with a median for the 26 ALMA-detected sources of $5.9 \times 10^{10} M_{\odot}$. This may be compared to the values derived by Scoville et al. (2014) for their mid- plus high-redshft sample galaxy stacks from the COSMOS field, which cover a similar redshift range to our sources. Their "IR Bright" sample has a median ISM mass of $(11.91 \pm 0.77) \times 10^{10} M_{\odot}$, about twice that of our sample.

\subsection{SED Fits}

The SEDs of most of the red WISE-NVSS sources are dominated by warm dust in the MIR with a strong decline into the submillimeter, and many of them display spectroscopic evidence of an obscured, high excitation AGN. The minimum AGN luminosity can be reasonably well determined from the warm dust emission that dominates the WISE data. The total $1-000 \mu \mathrm{m}$ luminosity is less well constrained, however, for most sources, because we lack data in the rest-frame FIR. We can model the emission by assuming a dominant dust temperature, $T_{\mathrm{d}}$, for the cooler dust that peaks in the FIR wavelength range, but the luminosity of such a component depends on the fourth power of $T_{\mathrm{d}}$, and is uncertain by $2-3$ dex without measurements at the FIR peak of the dust SED.

Two sources have available Herschel data, W1500-0649 and W2059-3541, and for these we find that the SED shapes fall well below the SEDs of M82 and Arp 220 longward of the MIR, resembling the radio-blind-selected Hot DOGs (Figure 4). We have constructed radiative transfer (RT) models for W1500-0649 $(z=1.50)$, which has the most detections (seven bands in total) that include good constraints on the peak of the SED, in order to provide some insights into the nature of these sources. For the remaining sample there are insufficient data points to justify RT models, therefore we use a parametric "torus" model plus modified blackbody (BB) fits for the cooler dust.

\subsubsection{RT Model for WISE 1500-0649}

We have constructed example models for W1500-0649 using both a tapered disk model (Efstathiou \& RowanRobinson 1995) for the torus (see also Efstathiou et al. 2013) and the two-phase clumpy torus models of Stalevski et al. (2012). For the cooler dust component radiating in the FIR we use the starburst models of Efstathiou et al. (2000) which were revised by Efstathiou \& Siebenmorgen (2009).

The tapered disk models have four parameters plus a normalization factor and the starburst models have three parameters plus a different normalization factor. In the tapered disc AGN models we fix the opening angle of the torus $\Theta_{0}$ at $45^{\circ}$ and vary the equatorial optical depth of the torus $\tau_{\mathrm{uv}}^{\mathrm{eq}}$ in the range $250-1250\left(A_{V} \approx 50-250\right)$, the inclination $i$ in the range $45^{\circ}-90^{\circ}$, and the ratio of outer torus radius to inner radius $r_{2} / r_{1}$ from 20 to 160 . In the starburst models we fix the initial optical depth of the molecular clouds $\tau_{V}$ at 75 (which is the average value in the model grid) and the e-folding time of the starburst at $20 \mathrm{Myr}$, which from previous work appears to be a reasonable timescale for starbursts (e.g., Efstathiou et al. 2000). In the starburst models we only vary the age of the starburst $t_{*}$.

We use a standard $\chi^{2}$ minimization technique to find the model parameters that best fit the data. The best tapered disk fit is shown in Figure 6-top (the AGN torus model is plotted with a blue dotted line and the starburst model with a red dashed line) and assumes the following parameters: $\tau_{\mathrm{uv}}^{\mathrm{eq}}=500, r_{2} /$ $r_{1}=160, i=54^{\circ}$, and $t^{*}=15 \mathrm{Myr}$.

The AGN torus has a derived luminosity of $1.9 \times 10^{13} L_{\odot}$. This needs to be multiplied by the anisotropy correction factor A (Efstathiou 2006), which for this particular combination of parameters is 0.84 , to give an AGN luminosity of $1.6 \times 10^{13} L_{\odot}$. The starburst luminosity is $1.8 \times 10^{13} L_{\odot}$ so the total luminosity of the system is predicted to be $3.4 \times 10^{13} L_{\odot}$.

In Figure 6-bottom we show the SED of W1500-0649 fitted with the two-phase clumpy torus models of Stalevski et al. (2012) in combination with the starburst models of Efstathiou \& Siebenmorgen (2009). We find that a good fit can be obtained with a torus that assumes a half-opening angle of $50^{\circ}$ and an inclination of $90^{\circ}$. The AGN luminosity is predicted to be $1.2 \times 10^{13} L_{\odot}$ and the starburst luminosity $2.4 \times 10^{13} L_{\odot}$. The starburst is still predicted to be a young system with an age of 10 Myr. We conclude that, irrespective of the uncertain dust 

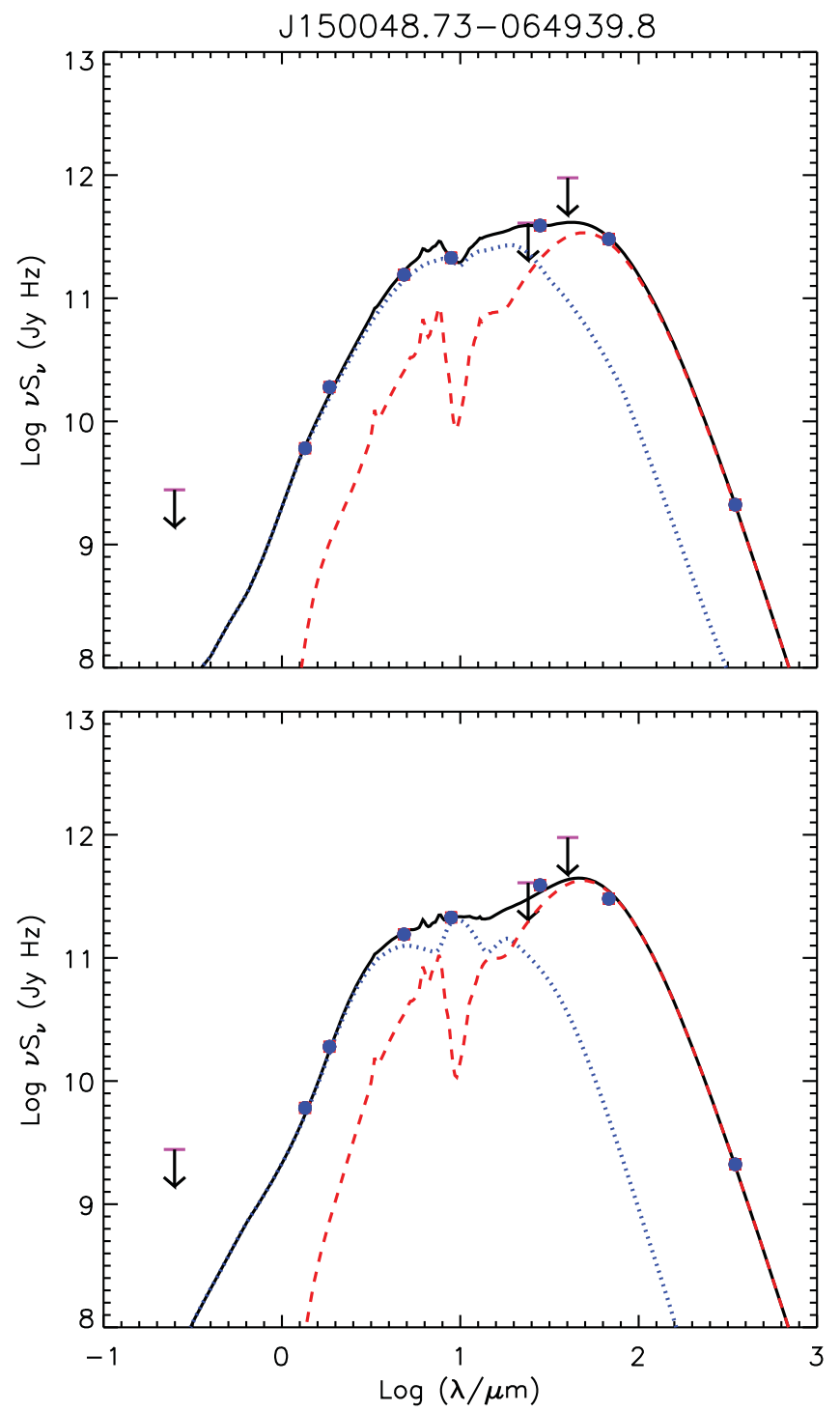

Figure 6. Best-fit radiative transfer models for WISEJ150048.73-064939.8. Top: AGN tapered disk model of Efstathiou \& Rowan-Robinson (1995) which has $\tau_{\mathrm{uv}}^{\mathrm{eq}}=500, r_{2} / r_{1}=160, i=54^{\circ}$, and $t_{*}=15 \mathrm{Myr}$. Bottom: AGN clumpy torus model of Stalevski et al. (2012) (blue dotted lines). The starburst models (red dashed lines) are from Efstathiou \& Siebenmorgen (2009).

geometry, in W1500-0649 the AGN and the starburst emit comparable luminosity.

\subsubsection{Three-component SED Fits}

We model the full sample by fitting the minimum number of simple spectral components to the SEDs that will define a reasonable maximum SFR that is consistent with the observed data, and to provide minimum and maximum estimates of the luminosity of the AGN-heated dust. A third SED component in the optical is used to estimate stellar mass, and we assume the AGN is completely obscured in the optical-UV. The results are presented in Tables 3-6 and illustrated in Figure 8. A more thorough analysis of the SEDs using RT models for the full sample is in preparation by A. Efstathiou et al.

The method fits a parameterized "torus" model to the MIR data and a modified blackbody (BB) to the longer wavelength SED. The "torus" model could describe a classical torus, or some other dusty structure heated by the AGN, including a spherical "cocoon" with $100 \%$ covering factor of warm dust, $\Omega_{\mathrm{WD}}$. For the BB component the characteristic dust temperature is undefined for most sources, therefore we construct four models with different fixed dust temperatures. Modeling a range of dust temperatures within an individual source is not justified by the available data points. We do not expect all sources to be fitted well for each of these temperature choices, particularly the higher values, and we carry forward into the analysis only those SED fits that are viable.

The methodology follows Sajina et al. (2012) but is simplified from their four dust components to only two, due to the limited SED data available for our sample. The AGN MIR emission is modeled with a parameterization that is consistent with the clumpy torus models of Nenkova et al. (2008). It has the functional form

$$
f_{\nu}=\frac{\nu}{\left(\frac{\nu}{\nu_{0}}\right)^{\alpha} e^{0.5 \nu}+\left(\frac{\nu}{\nu_{0}}\right)^{-0.5}+\left(\frac{\nu}{\nu_{0}}\right)^{-3}},
$$

where $\alpha$ and $\nu_{0}$ are free parameters. The MIR fit is phenomenological and so it is not characterized by specific values for the dust temperature range, orientation, torus size, or optical depth. We refer to this component henceforth as the "AGN" component. We emphasize that it could represent a structure of different shape than a classical torus, such as a more spherical cocoon, or a dusty narrow-line region (NLR) or polar wind. We also do not rule out in our later discussion that some of this warm emission could be contributed by a young compact starburst.

The modified blackbody component has a fixed dust temperature and emissivity, $\beta$. With only the single ALMA data point longward of $22 \mu \mathrm{m}$ for most sources, it is impossible to fit the long-wavelength portion of the SED using free parameters, therefore a single graybody is the best approximation. This component may represent dust heated by star formation, or dust heated by the AGN that is cooler than the "AGN component," and of course would in reality it have a wide range of dust temperatures compared to the single value used here. The fit to the MIR region of the spectrum with the AGN component varies very little as the BB component temperature is changed, because the AGN component is very well defined by the WISE data on the short-wavelength side. For the four sources without measured redshift we assume $z=2$.

For the stellar luminosity and mass we use either a 100 or $600 \mathrm{Myr}$ stellar population from Maraston (2005). This is constrained only by the $R$-band data point in most cases, except for the two SDSS-detected sources, and the shape of the MIR SED at the shortest wavelengths.

We have made four fits to each source, each with a different temperature for the $\mathrm{BB}$ component. We have allowed the temperatures to take on a wide range so that we can interpret the luminosity as arising from disk-like distributed star formation (the coolest dust temperature, $30 \mathrm{~K}$ ), a starburst similar to those found in local LIRGs and ULIRGs (50 K) (Melbourne et al. 2012; Bendo et al. 2015), and two additional warmer temperatures that might be appropriate for very young and compact starbursts and/or for additional AGN-heated dust (Wilson et al. 2014). For $\beta$ we have selected a value of 1.5, consistent with the range of values found in the literature. For the $50 \mathrm{~K}$ model only, we instead used a value of $\beta=2$, 
Table 4

Minimum and Maximum Model Ranges in Luminosity, Mass, SFR, and Accretion Rate $(\dot{M})$

\begin{tabular}{|c|c|c|c|c|c|c|c|}
\hline & & $\mathrm{BB}$ & Total & Total-Best & $\mathrm{BB}$ & Total & Total-Best \\
\hline Parameter & $\mathrm{AGN}$ & & $870 \mu \mathrm{m}$ Detected & & \multicolumn{3}{|c|}{$870 \mu \mathrm{m}$ Not Detected } \\
\hline Min. Value & W0652-2006 & W0612-0622 & W0652-2006 & W0612-0622 & W2000-2802 & W0702-2808 & W0702-2808 \\
\hline Max. Value & $\mathrm{W} 1521+0017$ & W0439-2159 & W0439-2159 & W2021-2611 & W0525-3225 & W1702-3225 & W1702-3225 \\
\hline Redshift & $0.60-2.63$ & $0.47-2.82$ & $0.60-2.82$ & $0.47-2.28$ & $2.28-1.69$ & $0.94-2.85$ & $0.94-2.85$ \\
\hline $\log L\left(L_{\odot}\right)$ & $11.58-13.61$ & $10.89-14.17$ & $11.71-14.24$ & $12.07-14.15$ & $<10.72-<13.75$ & $<12.09-<12.46$ & $<12.41-<13.81$ \\
\hline $\operatorname{SFR}\left(M_{\odot} \mathrm{yr}^{-1}\right)$ & $\cdots$ & $13.5-25700$ & $22-25700$ & $\ldots^{a}$ & $<9-<9800$ & $<22-<4360$ & $\ldots^{\mathrm{a}}$ \\
\hline $\log M_{\mathrm{BH}}\left(M_{\odot}\right)^{\mathrm{b}}$ & $7.66-9.69$ & $\ldots$ & $\ldots^{\mathrm{c}}$ & $8.11-10.23$ & $\ldots$ & $\ldots^{\mathrm{c}}$ & $<8.49-<9.89$ \\
\hline$\dot{M}\left(M_{\odot} \mathrm{yr}^{-1}\right)^{\mathrm{b}}$ & $0.24-26$ & $\ldots$ & $\ldots^{\mathrm{c}}$ & $0.7-90$ & $\ldots$ & $\ldots^{\mathrm{c}}$ & $<1.6-<41$ \\
\hline
\end{tabular}

Notes.

${ }^{\mathrm{a}}$ No star formation is present in the Total-Best model by definition, since it is designed to resemble the intrinsic AGN SEDs.

${ }^{\mathrm{b}} M_{\mathrm{BH}}$ and accretion rate are directly proportional to the luminosity since we have assumed a fixed Eddington ratio and accretion efficiency.

${ }^{c}$ Some Total $=$ AGN + BB fits are inconsistent with a torus-like or intrinsic AGN SED shape; the Total-Best model is the preferable maximal fit for an AGN.

consistent with the largest values found in the literature, in order to illustrate that the uncertainties due to the unknown dust temperature distribution in each source far outweigh the effect of the choice of $\beta$. This may be seen by comparing the $30 \mathrm{~K}$ and $50 \mathrm{~K}$ model fits in Figure 8. We do not expect any of these fits to be unique for any of our sources; instead we use them to constrain the range of plausible AGN and starburst luminosities.

To select appropriate dust temperatures for the two warmer BB models, we ran a set of models where $T_{\mathrm{d}}$ was allowed to be a free parameter for the two sources that have Herschel data, and the results are shown in Figure 7-right, compared to the 30 and $50 \mathrm{~K}$ fixed- $T$ fits in the left panels. For W1500-0649, which was fitted in the previous section with RT models, there are two plausible fits. The $50 \mathrm{~K} \mathrm{BB}$ model is reasonable, although the IRAS $60 \mu \mathrm{m}$ limit is slightly exceeded. This fit requires a large fraction of the FIR emission to be explained by the warm AGN component (dashed cyan line). The best fit free$T_{\mathrm{d}}$ model has $T_{\mathrm{d}}=89 \mathrm{~K}$ and provides a total MIRsubmillimeter luminosity of $\log \left(L_{\text {Total }} / L_{\odot}\right)=13.52$, and a division between the AGN and BB components of 1.17 and $2.14 \times 10^{13} L_{\odot}$. This is a very similar result to the RT model. For W2059-3541 a dust temperature greater than $50 \mathrm{~K}$ is required to match the $70 \mu \mathrm{m}$ PACS data. The best-fit free- $T_{\mathrm{d}}$ model has $T_{\mathrm{d}}=120 \mathrm{~K}$, with a total MIR-submillimeter luminosity of $\log \left(L_{\mathrm{bol}} / L_{\odot}\right)<13.33$ (the $870 \mu$ m measurement is a non-detection for this source), assuming $\beta=1.5$.

For the remainder of the sample, for which free- $T_{\mathrm{d}}$ fits are insufficiently constrained, we have adopted the two dust temperature values that have fitted W1500-0649 and W2059-3541 successfully: $90 \mathrm{~K}$ (rounding up from $89 \mathrm{~K}$ ) and $120 \mathrm{~K}$, for the two warmest of the four BB models. The two fits with the cooler dust temperatures, 30 and $50 \mathrm{~K}$, are shown in the left-hand panels of Figure 8, while the fits with the two warmer temperatures, 90 and $120 \mathrm{~K}$, are shown in the right-hand panels. The results are tabulated in Table 3. Since the AGN luminosities do not vary much between the four models, we list only the minimum and maximum AGN component luminosities in columns 3 and 4 of Table 3: $L_{\mathrm{AGN}-}$ Min and $L_{\mathrm{AGN}-\mathrm{Max}}$.

Many of the BB fits are not successful in fitting all the data points; in particular the $120 \mathrm{~K}$ fits exceed the IRAS limits for many sources. Also the stellar component is not always fitted well by the code. We have not attempted to refine the fitting procedures to improve this situation because the current optical photometry is inadequate for this purpose. Only fits judged to be acceptable are tabulated and used in the subsequent analysis.

For the two warmer BB models, the IRAS $60 \mu \mathrm{m}$ limit (and occasionally the $100 \mu \mathrm{m}$ limit) is helpful in constraining the dust temperature of the fits for many sources. Four sources fail to find a reasonable fit for the IRAS limits with $T_{\mathrm{d}}=90 \mathrm{~K}$, while for 29 sources, the IRAS $60 \mu \mathrm{m}$ limit rules out the $120 \mathrm{~K}$ model. In one case, W1500-0649, the $120 \mathrm{~K}$ model is ruled out not by IRAS but by ALMA and Herschel. In some cases the low-temperature BB models fail to allow the overall model to fit the WISE data; these sources are better fit with one or both of the two warmer models.

We derive SFRs from the BB components of the models using the Kennicutt (1998) conversion from far-infrared luminosity to SFR:

$$
\mathrm{SFR}=4.4 \times 10^{-37} L_{\mathrm{BB}}(\mathrm{W}) M_{\odot} \mathrm{yr}^{-1} .
$$

The SFR results are given in Table 6, columns 3-6, for those models that achieved successful fits.

We also list in Table 3 columns 9 and 10 the minimum and maximum summed luminosity from the up to four viable $L_{\text {Total }}$ $\left(=L_{\mathrm{AGN}}+L_{\mathrm{BB}}\right)$ models, $L_{\text {Total-Min }}$ and $L_{\text {Total-Max }}$, for each source. In addition we list $L_{\text {Total-Best }}$ in column 11 , which represents the $L_{\text {Total }}$ fit that best resembles the SED shapes of the two sources with well fitted SEDs: W1500-0649 and W2059-3541, and the Hot DOGs with well-sampled SEDs (Figure 4). The best-fit model is indicated in column 12. In some cases the best match is derived from the average of two of the $L_{\text {Total }}$ models.

The luminosities derived for the AGN from the AGN model component range from $\log \left(L_{\mathrm{AGN}-\mathrm{Min}} / L_{\odot}\right)=11.58$ to $\log \left(L_{\mathrm{AGN}-}\right.$ $\left.\operatorname{Max} / L_{\odot}\right)=13.61$. We also consider a maximum AGN luminosity derived from the Total-Best models: log ( $L_{\text {Total }}$ $\left.{ }_{- \text {Best }} / L_{\odot}\right)=12.01-14.15$. The BB component luminosity depends strongly on assumed dust temperature. Considering all acceptable fits to the SEDs for all of the $870 \mu \mathrm{m}$ detected sources, the acceptable range in $\log \left(L_{\mathrm{BB}} / L_{\odot}\right)$ is $10.89-14.17$, and the corresponding SFR range is $13.5-25700 M_{\odot} \mathrm{yr}^{-1}$. The total summed AGN + BB luminosity range is $\log \left(L_{\text {Total }} /\right.$ $\left.L_{\odot}\right)=11.71-14.24$. These results are summarized in Table 4.

\subsection{Stellar Masses}

The stellar population fit can be used to constrain the host galaxy mass from the rest-frame $H$-band absolute magnitude of the fitted stellar component. The host galaxy mass has only a 
Table 5

Masses and Accretion Rates

\begin{tabular}{|c|c|c|c|c|c|c|c|c|c|c|c|c|c|}
\hline \multirow[t]{2}{*}{ WISE Name } & \multirow[t]{2}{*}{ Redshift } & \multicolumn{3}{|c|}{$\begin{array}{c}\log M_{\mathrm{BH}} \\
\left(M_{\odot}\right)\end{array}$} & \multicolumn{2}{|c|}{$\begin{array}{c}\log M_{\mathrm{BH}} \\
\text { Kim, et al. } \\
\left(M_{\odot}\right)\end{array}$} & \multicolumn{3}{|c|}{$\begin{array}{l}\text { Accretion Rates } \\
\qquad\left(M_{\odot} \mathrm{yr}^{-1}\right)\end{array}$} & \multirow[t]{2}{*}{$\begin{array}{c}\text { Absolute } \\
H \text {-Magnitude }\end{array}$} & \multirow[t]{2}{*}{$\begin{array}{c}\log M_{\text {stars }} \\
\left(M_{\odot}\right)\end{array}$} & \multicolumn{2}{|c|}{$\begin{array}{c}\log M_{\text {ISM }} \\
\left(M_{\odot}\right)\end{array}$} \\
\hline & & Min. & Max. & Total-Best & Lower & Upper & Min. & Max. & Total-Best & & & $30 \mathrm{~K}$ & $90 \mathrm{~K}$ \\
\hline W0304-3108 & 1.54 & 9.09 & 9.24 & 9.44 & $\ldots$ & $\ldots$ & 6.52 & 9.20 & 14.50 & -24.5 & 10.6 & 10.83 & 10.20 \\
\hline W0306-3353 & 0.78 & 8.27 & 8.31 & 8.61 & $\ldots$ & $\ldots$ & 0.99 & 1.08 & 2.18 & -23.9 & 10.4 & 10.51 & 9.96 \\
\hline W0354-3308 & 1.37 & 8.78 & 8.80 & $<9.04$ & $\ldots$ & $\ldots$ & 3.19 & 3.34 & $<5.82$ & -23.6 & 10.2 & $<10.52$ & $<9.91$ \\
\hline W0404-2436 & 1.26 & 8.66 & 8.71 & 9.19 & $\ldots$ & $\ldots$ & 2.42 & 2.72 & 8.14 & -25.1 & 10.8 & 10.86 & 10.26 \\
\hline W0409-1837 & 0.67 & 8.81 & 8.82 & $<8.98$ & $\ldots$ & $\ldots$ & 3.42 & 3.50 & $<5.10$ & -22.6 & 9.8 & $<10.44$ & $<9.90$ \\
\hline W0417-2816 & 0.94 & 8.03 & 8.09 & 8.55 & 9.06 & 9.72 & 0.57 & 0.65 & 1.87 & -22.9 & 9.9 & 10.63 & 10.06 \\
\hline W0439-3159 & 2.82 & 9.52 & 9.53 & 9.78 & $\ldots$ & $\ldots$ & 17.54 & 17.95 & 31.56 & -23.6 & 10.3 & 11.15 & 10.38 \\
\hline W0519-0813 & 2.05 & 9.09 & 9.10 & $<9.26$ & $\ldots$ & $\ldots$ & 6.52 & 6.67 & $<9.72$ & $>-23.1$ & $<10.0$ & $<10.53$ & $<9.85$ \\
\hline W0525-3614 & 1.69 & 8.59 & 8.68 & $<9.02$ & $\ldots$ & $\ldots$ & 2.06 & 2.54 & $<5.58$ & -23.5 & 10.2 & $<10.59$ & $<9.94$ \\
\hline W0526-3225 & 1.98 & 9.52 & 9.62 & 10.03 & $\ldots$ & $\ldots$ & 17.54 & 22.08 & 56.80 & -24.1 & 10.4 & 11.64 & 10.96 \\
\hline W0536-2703 & 1.79 & 9.13 & 9.13 & 9.38 & $\ldots$ & $\ldots$ & 7.14 & 7.14 & 12.69 & -23.7 & 10.3 & 10.82 & 10.16 \\
\hline W0549-3739 & 1.71 & 8.64 & 8.64 & 9.08 & $\ldots$ & $\ldots$ & 2.31 & 2.31 & 6.42 & $>-22.7$ & $<9.9$ & 10.69 & 10.04 \\
\hline W0612-0622 & 0.47 & 8.11 & 8.12 & 8.51 & $\ldots$ & $\ldots$ & 0.68 & 0.70 & 1.71 & -23.8 & 10.2 & 10.53 & 10.01 \\
\hline W0613-3407 & 2.18 & 9.28 & 9.28 & $<9.42$ & 9.07 & 9.46 & 10.09 & 10.09 & $<14.02$ & $>-22.6$ & $<9.8$ & $<10.64$ & $<9.94$ \\
\hline W0614-0936 & $\ldots^{\mathrm{a}}$ & 9.02 & 9.03 & $<9.25$ & $\ldots$ & $\ldots$ & 5.55 & 5.68 & $<9.51$ & -22.9 & $<9.9$ & $<10.64$ & $<9.96$ \\
\hline W0630-2120 & 1.44 & 8.60 & 8.65 & 8.90 & $\ldots$ & $\ldots$ & 2.11 & 2.37 & 4.17 & -24.0 & 10.4 & 11.08 & 10.46 \\
\hline W0642-2728 & 1.34 & 8.44 & 8.44 & 8.74 & , & & 1.46 & 1.46 & 2.88 & -24.1 & 10.4 & 10.72 & 10.11 \\
\hline W0652-2006 & 0.60 & 7.66 & 7.67 & 8.20 & 8.85 & 9.61 & 0.24 & 0.25 & 0.84 & $>-21.3$ & $<9.2$ & 10.70 & 10.16 \\
\hline W0702-2808 & 0.94 & 8.12 & 8.12 & $<8.49$ & 8.92 & 9.73 & 0.70 & 0.70 & $<1.64$ & -21.9 & 9.5 & $<10.58$ & $<10.01$ \\
\hline W0714-3635 & 0.88 & 7.96 & 7.97 & 8.49 & 9.26 & 9.34 & 0.48 & 0.49 & 1.64 & -21.7 & 9.5 & 10.69 & 10.13 \\
\hline W0719-3349 & 1.63 & 8.65 & 8.65 & 9.09 & $\ldots$ & $\ldots$ & 2.37 & 2.37 & 6.47 & -22.5 & 9.8 & 10.51 & 9.74 \\
\hline W0811-2225 & 1.11 & 8.71 & 8.71 & $<9.02$ & $\ldots$ & $\ldots$ & 2.72 & 2.72 & $<5.56$ & -24.0 & 10.4 & $<10.61$ & $<10.02$ \\
\hline W0823-0624 & 1.75 & 9.19 & 9.30 & $<9.43$ & $\ldots$ & $\ldots$ & 8.20 & 10.57 & $<14.23$ & -24.2 & 10.5 & $<10.64$ & $<9.99$ \\
\hline W1308-3447 & 1.65 & 9.07 & 9.07 & 9.23 & $\ldots$ & $\ldots$ & 6.22 & 6.22 & 9.07 & -24.4 & 10.6 & 10.53 & 9.89 \\
\hline W1343-1136 & 2.49 & 9.08 & 9.10 & 9.35 & $\ldots$ & $\ldots$ & 6.37 & 6.67 & 11.81 & -25.7 & 11.1 & 10.74 & 10.00 \\
\hline W1400-2919 & 1.67 & 9.18 & 9.19 & $<9.51$ & $\ldots$ & $\ldots$ & 8.02 & 8.20 & $<17.22$ & -24.8 & 10.7 & $<10.09$ & $<9.44$ \\
\hline W1412-2020 & 1.82 & 9.10 & 9.25 & 9.44 & $\ldots$ & $\ldots$ & 6.67 & 9.42 & 14.72 & $>-24.7$ & $<10.7$ & 10.80 & 10.14 \\
\hline W1434-0235 & 1.92 & 8.97 & 8.97 & $<9.11$ & $\ldots$ & $\ldots$ & 4.94 & 4.94 & $<6.82$ & -24.5 & 10.6 & $<10.34$ & $<9.67$ \\
\hline W1439-3725 & 1.19 & 8.37 & 8.37 & $<8.66$ & $\ldots$ & $\ldots$ & 1.24 & 1.24 & $<2.40$ & $>-23.2$ & $<10.1$ & $<10.21$ & $<9.61$ \\
\hline W1500-0649 & 1.50 & 9.15 & 9.58 & 9.60 & $\ldots$ & $\ldots$ & 7.48 & 20.14 & 21.10 & $>-23.2$ & $<10.1$ & 11.18 & 10.56 \\
\hline W1510-2203 & 0.95 & 8.68 & 8.69 & $<8.84$ & $\ldots$ & $\ldots$ & 2.54 & 2.59 & $<3.68$ & $>-23.1$ & $<10.0$ & $<10.23$ & $<9.66$ \\
\hline W1513-2210 & 2.20 & 9.34 & 9.34 & 9.62 & $\ldots$ & $\ldots$ & 11.59 & 11.59 & 22.16 & $>-23.9$ & $<10.4$ & 11.07 & 10.36 \\
\hline W1514-3411 & 1.09 & 8.52 & 8.56 & $<8.78$ & $\ldots$ & $\ldots$ & 1.75 & 1.92 & $<3.16$ & $>-23.6$ & $<10.2$ & $<10.25$ & $<9.67$ \\
\hline $\mathrm{W} 1521+0017$ & 2.63 & 9.69 & 9.69 & 9.73 & $\ldots$ & $\ldots$ & 25.94 & 25.94 & 28.66 & $>-26.1$ & $<11.2$ & 11.75 & 11.20 \\
\hline W1541-1144 & 1.58 & 8.89 & 9.02 & 9.09 & $\ldots$ & $\ldots$ & 4.11 & 5.55 & 6.48 & $>-23.5$ & $<10.2$ & 10.46 & 9.83 \\
\hline W1634-1721 & 2.08 & 8.91 & 8.91 & $<9.06$ & $\ldots$ & $\ldots$ & 4.31 & 4.31 & $<6.14$ & $>-25.1$ & $<10.8$ & $<10.31$ & $<9.62$ \\
\hline W1641-0548 & 1.84 & 9.02 & 9.17 & 9.38 & $\ldots$ & $\ldots$ & 5.55 & 7.83 & 12.66 & $>-26.4$ & $<11.3$ & 10.75 & 10.09 \\
\hline W1653-0102 & 2.02 & 9.08 & 9.08 & $<9.18$ & $\ldots$ & $\ldots$ & 6.37 & 6.37 & $<8.00$ & $>-25.4$ & $<11.0$ & $<10.28$ & $<9.60$ \\
\hline W1657-1740 & $\ldots^{\mathrm{a}}$ & 9.24 & 9.25 & $<9.68$ & $\ldots$ & $\ldots$ & 9.20 & 9.42 & $<25.42$ & $>-25.1$ & $<10.8$ & $<10.28$ & $<9.60$ \\
\hline W1702-0811 & 2.85 & 9.68 & 9.68 & $<9.89$ & $\ldots$ & $\ldots$ & 25.35 & 25.35 & $<41.35$ & $>-25.2$ & $<10.9$ & $<10.38$ & $<9.61$ \\
\hline W1703-0517 & 1.80 & 9.19 & 9.59 & 9.68 & $\ldots$ & $\ldots$ & 8.20 & 20.61 & 25.62 & $>-25.1$ & $<10.8$ & 9.92 & 9.16 \\
\hline W1707-0939 & $\ldots^{\mathrm{a}}$ & 9.06 & 9.12 & $<9.64$ & $\cdots$ & $\cdots$ & 6.08 & 6.98 & $<23.22$ & $>-25.1$ & $<10.8$ & $<10.39$ & $<9.71$ \\
\hline W1936-3354 & 2.24 & 9.13 & 9.26 & 9.86 & $\ldots$ & $\ldots$ & 7.14 & 9.64 & 38.33 & $>-24.5$ & $<10.6$ & 10.97 & 10.32 \\
\hline W1951-0420 & 1.58 & 8.88 & 8.91 & $<9.62$ & $\ldots$ & $\ldots$ & 4.02 & 4.31 & $<21.97$ & $>-24.9$ & $<10.7$ & $<10.40$ & $<9.76$ \\
\hline W1958-0746 & 1.80 & 9.06 & 9.06 & $<9.18$ & 9.53 & $\ldots$ & 6.08 & 6.08 & $<8.00$ & $>-24.7$ & $<10.7$ & $<10.36$ & $<9.70$ \\
\hline W2000-2802 & 2.28 & 8.83 & 8.98 & $<9.04$ & $\ldots$ & $\ldots$ & 3.58 & 5.06 & $<5.81$ & $>-23.6$ & $<10.2$ & $<10.36$ & $<9.65$ \\
\hline W2021-2611 & 2.44 & 9.05 & 9.59 & 10.23 & $\ldots$ & $\ldots$ & 5.94 & 20.61 & 89.96 & $>-25.4$ & $<10.9$ & 11.24 & 10.56 \\
\hline W2040-3904 & $\ldots^{\mathrm{a}}$ & 9.00 & 9.23 & 9.57 & $\ldots$ & $\ldots$ & 5.30 & 8.99 & 19.81 & $>-24.8$ & $<10.7$ & 11.09 & 10.41 \\
\hline W2059-3541 & 2.38 & 9.23 & 9.45 & $<9.50$ & 9.07 & 9.10 & 8.99 & 14.93 & $<16.76$ & $>-25.2$ & $<10.9$ & $<10.35$ & $<9.62$ \\
\hline
\end{tabular}

Note.

${ }^{\mathrm{a}}$ Redshift assumed to be 2 if no spectroscopic redshift available.

small dependence on the stellar population selected by the fitting code. The masses do not depend on the dust temperature assumed for the BB dust component because the optical fit is dominated by the AGN component fitted to the WISE data. In a few cases, a self-consistent fit could not be obtained, indicating some possible confusion in the $R$-band data point. The $H$-band absolute magnitudes are upper limits in more than half the sample since there is no $R$-band measurement or detection, therefore most of the stellar mass estimates in columns 11 and 12 of Table 5 are also upper limits. The stellar masses derived from the models range from $\log \left(M_{\text {stars }} / M_{\odot}\right)=9.83$ to 11.09 for $R$-band detected sources. The upper limits ranges from log $\left(M_{\text {stars }} / M_{\odot}\right)<9.82$ to $<11.34$.

The modeled intrinsic $H$-band absolute magnitudes and the derived stellar masses are highly uncertain and should be viewed as indicative only. We have no measurement of 
Table 6

Star Formation Rates and Gas Depletion Timescales

\begin{tabular}{|c|c|c|c|c|c|c|c|c|}
\hline \multirow[t]{2}{*}{ WISE Name } & \multirow[t]{2}{*}{ Redshift } & \multicolumn{4}{|c|}{$\begin{array}{l}\log \mathrm{SFR} \\
\left(L_{\odot} \mathrm{yr}^{-1}\right)\end{array}$} & \multicolumn{3}{|c|}{$\begin{array}{c}\log (\text { Gas Depletion Time }) \\
(\mathrm{yr})\end{array}$} \\
\hline & & $30 \mathrm{~K}$ & $50 \mathrm{~K}$ & $90 \mathrm{~K}$ & $120 \mathrm{~K}$ & $30 \mathrm{~K}$ & $50 \mathrm{~K}$ & $90 \mathrm{~K}$ \\
\hline W0304-3108 & 1.54 & 1.73 & 2.70 & 3.16 & $\ldots$ & 9.10 & 7.81 & 7.04 \\
\hline W0306-3353 & 0.78 & 1.22 & 2.31 & 2.52 & $\ldots$ & 9.29 & 7.93 & 7.44 \\
\hline W0354-3308 & 1.37 & $<1.38$ & $<2.38$ & $<2.83$ & $\ldots$ & $<9.14$ & $<7.83$ & $<7.08$ \\
\hline W0404-2436 & 1.26 & 1.70 & 2.72 & 3.17 & $\ldots$ & 9.16 & 7.84 & 7.09 \\
\hline W0409-1837 & 0.67 & $\ldots$ & $<2.23$ & $<2.64$ & $\ldots$ & $\ldots$ & $<7.94$ & $<7.26$ \\
\hline W0417-2816 & 0.94 & 1.72 & 2.79 & 3.22 & $\ldots$ & 8.91 & 7.56 & 6.84 \\
\hline W0439-3159 & 2.82 & 2.28 & 3.08 & 3.57 & 4.41 & 8.87 & 7.66 & 6.81 \\
\hline W0519-0813 & 2.05 & $<1.54$ & $<2.44$ & $<2.92$ & $<3.82$ & $<8.99$ & $<7.74$ & $<6.93$ \\
\hline W0525-3614 & 1.69 & $<1.53$ & $<2.49$ & $<2.95$ & $<3.99$ & $<9.06$ & $<7.77$ & $<6.99$ \\
\hline W0526-3225 & 1.98 & 2.64 & 3.55 & 4.03 & $\ldots$ & 9.00 & 7.74 & 6.93 \\
\hline W0536-2703 & 1.79 & $\ldots$ & $\ldots$ & 3.18 & $\ldots$ & $\ldots$ & $\ldots$ & 6.98 \\
\hline W0549-3739 & 1.71 & $\ldots$ & 2.58 & 3.05 & $\ldots$ & 9.06 & 7.78 & 6.99 \\
\hline W0612-0622 & 0.47 & 1.13 & 2.29 & 2.00 & $\ldots$ & 9.40 & 7.99 & 7.57 \\
\hline W0613-3407 & 2.18 & $<1.67$ & $<2.55$ & $<3.03$ & $<3.92$ & $<8.97$ & $<7.73$ & $<6.91$ \\
\hline W0614-0936 & $\ldots^{a}$ & $<1.64$ & $<2.55$ & $<3.02$ & $<3.93$ & $<9.00$ & $<7.74$ & $<6.94$ \\
\hline W0630-2120 & 1.44 & 1.96 & 2.95 & 3.41 & $\ldots$ & 9.12 & 7.82 & 7.05 \\
\hline W0642-2728 & 1.34 & 1.58 & 2.59 & $\ldots$ & $\ldots$ & 9.14 & 7.82 & $\ldots$ \\
\hline W0652-2006 & 0.60 & 1.35 & 2.48 & $\ldots$ & $\ldots$ & 9.35 & 7.96 & $\ldots$ \\
\hline W0702-2808 & 0.94 & $<1.34$ & $<2.41$ & $\ldots$ & $\ldots$ & $<9.24$ & $<7.89$ & $\cdots$ \\
\hline W0714-3635 & 0.88 & 1.42 & 2.50 & $\ldots$ & $\ldots$ & 9.27 & 7.91 & $\ldots$ \\
\hline W0719-3349 & 1.63 & $\ldots$ & 2.99 & 3.46 & $\ldots$ & $\ldots$ & 7.11 & 6.28 \\
\hline W0811-2225 & 1.11 & $\ldots$ & $\ldots$ & $<2.89$ & $\ldots$ & $\ldots$ & $\ldots$ & $<7.13$ \\
\hline W0823-0624 & 1.75 & $<1.60$ & $<2.54$ & $<3.00$ & $\ldots$ & $<9.04$ & $<7.77$ & $<6.99$ \\
\hline W1308-3447 & 1.65 & 1.46 & 2.42 & 2.89 & $\ldots$ & 9.07 & 7.78 & 7.00 \\
\hline W1343-1136 & 2.49 & $\ldots$ & 2.67 & 3.16 & 4.02 & $\ldots$ & 7.69 & 6.84 \\
\hline W1400-2919 & 1.67 & $<1.02$ & $<1.97$ & $\ldots$ & $<3.40$ & $<9.07$ & $<7.79$ & $\cdots$ \\
\hline W1412-2020 & 1.82 & 1.76 & 2.69 & 3.16 & $\ldots$ & 9.04 & 7.77 & 6.98 \\
\hline W1434-0235 & 1.92 & $<1.32$ & $<2.24$ & $<2.71$ & $<3.63$ & $<9.02$ & $<7.75$ & $<6.96$ \\
\hline W1439-3725 & 1.19 & $<1.03$ & $<2.05$ & $<2.50$ & $\ldots$ & $<9.18$ & $<7.86$ & $<7.11$ \\
\hline W1500-0649 & 1.50 & $\ldots$ & 3.08 & 3.57 & $\ldots$ & $\ldots$ & 7.79 & 6.99 \\
\hline W1510-2203 & 0.95 & $<0.98$ & $<2.05$ & $<2.47$ & $\ldots$ & $<9.25$ & $<7.90$ & $<7.19$ \\
\hline W1513-2210 & 2.20 & 2.10 & 2.98 & 3.46 & $\ldots$ & 8.97 & 7.72 & 6.90 \\
\hline W1514-3411 & 1.09 & $<1.05$ & $<2.10$ & $<2.53$ & $\ldots$ & $<9.20$ & $<7.86$ & $<7.14$ \\
\hline $\mathrm{W} 1521+0017$ & 2.63 & $\ldots$ & $\ldots$ & 2.87 & $\ldots$ & $\ldots$ & $\ldots$ & 8.33 \\
\hline W1541-1144 & 1.58 & 1.37 & 2.34 & 2.81 & $\ldots$ & 9.09 & 7.80 & 7.02 \\
\hline W1634-1721 & 2.08 & $<1.32$ & $<2.22$ & $<2.70$ & $<3.60$ & $<8.99$ & $<7.73$ & $<6.92$ \\
\hline W1641-0548 & 1.84 & $\ldots$ & 2.65 & 3.12 & $\ldots$ & $\ldots$ & 7.76 & 6.97 \\
\hline W1653-0102 & 2.02 & $<1.28$ & $<2.18$ & $<2.65$ & $<3.55$ & $<9.00$ & $<7.75$ & $<6.95$ \\
\hline W1657-1740 & $\ldots^{\mathrm{a}}$ & $<1.27$ & $<2.18$ & $<2.66$ & $<3.56$ & $<9.01$ & $<7.75$ & $<6.94$ \\
\hline W1702-0811 & 2.85 & $<1.52$ & $<2.31$ & $\ldots$ & $<3.64$ & $<8.86$ & $<7.66$ & $\ldots$ \\
\hline W1703-0517 & 1.80 & 1.36 & 2.29 & 2.72 & 3.69 & 8.56 & 7.23 & 6.44 \\
\hline W1707-0939 & $\ldots^{\mathrm{a}}$ & $<1.39$ & $<2.30$ & $<2.78$ & $<3.67$ & $<9.00$ & $<7.74$ & $<6.93$ \\
\hline W1936-3354 & 2.24 & 1.69 & 2.56 & $\ldots$ & 3.93 & 9.28 & 8.08 & $\cdots$ \\
\hline W1951-0420 & 1.58 & $<1.32$ & $<2.29$ & $\ldots$ & $>5.41$ & $<9.08$ & $<7.79$ & $\cdots$ \\
\hline W1958-0746 & 1.80 & $<1.32$ & $<2.25$ & $<2.72$ & $<3.65$ & $<9.04$ & $<7.77$ & $<6.98$ \\
\hline W2000-2802 & 2.28 & $<0.96$ & $<1.83$ & $<2.31$ & $<3.20$ & $<9.40$ & $<8.16$ & $<7.34$ \\
\hline W2021-2611 & 2.44 & 2.09 & 2.92 & $\ldots$ & 4.28 & 9.15 & 7.97 & 0.00 \\
\hline W2040-3904 & $\ldots^{\mathrm{a}}$ & 2.09 & 3.00 & 3.47 & $\ldots$ & 9.00 & 6.94 & $\ldots$ \\
\hline W2059-3541 & 2.38 & $\ldots$ & $\ldots$ & $<2.70$ & $<3.11$ & $\ldots$ & $\ldots$ & $<6.92$ \\
\hline
\end{tabular}

Note.

${ }^{\mathrm{a}} z=2$ assumed for sources without a spectroscopic redshift.

extinction for the optical or WISE data points, and no color information to help constrain the stellar populations. We also have little information on morphology, and therefore on the stellar components included in the flux density. It is also possible that some of the emission is scattered AGN light. The fitting of the stellar component will be greatly improved by the use of the $J$ and $K_{s}$ data, therefore we limit discussion of these results in this paper.

\subsection{Radio Powers, Radio Loudness and the q Parameter}

The rest-frame $3 \mathrm{GHz}$ radio powers in column 13 of Table 3 are found to lie in the range $\log \left(P_{3 \mathrm{GHz}} / \mathrm{W}\right.$ $\left.\mathrm{Hz}^{-1}\right)=24.74-27.33$, adopting a power-law spectral index $\alpha=-1.0\left(f_{\nu} \propto \nu^{\alpha}\right)$ for the $k$-correction to $3 \mathrm{GHz}$ rest frequency. The median/mean value is $25.97 / 26.05$. We also list the rest-frame (i.e., $k$-corrected) $q_{22}=\log \left(f_{22 \mu \mathrm{m}} / f_{1.4 \mathrm{GHz}}\right)$ values in column 14 . The MIR $k$-correction for $q$ obviously 

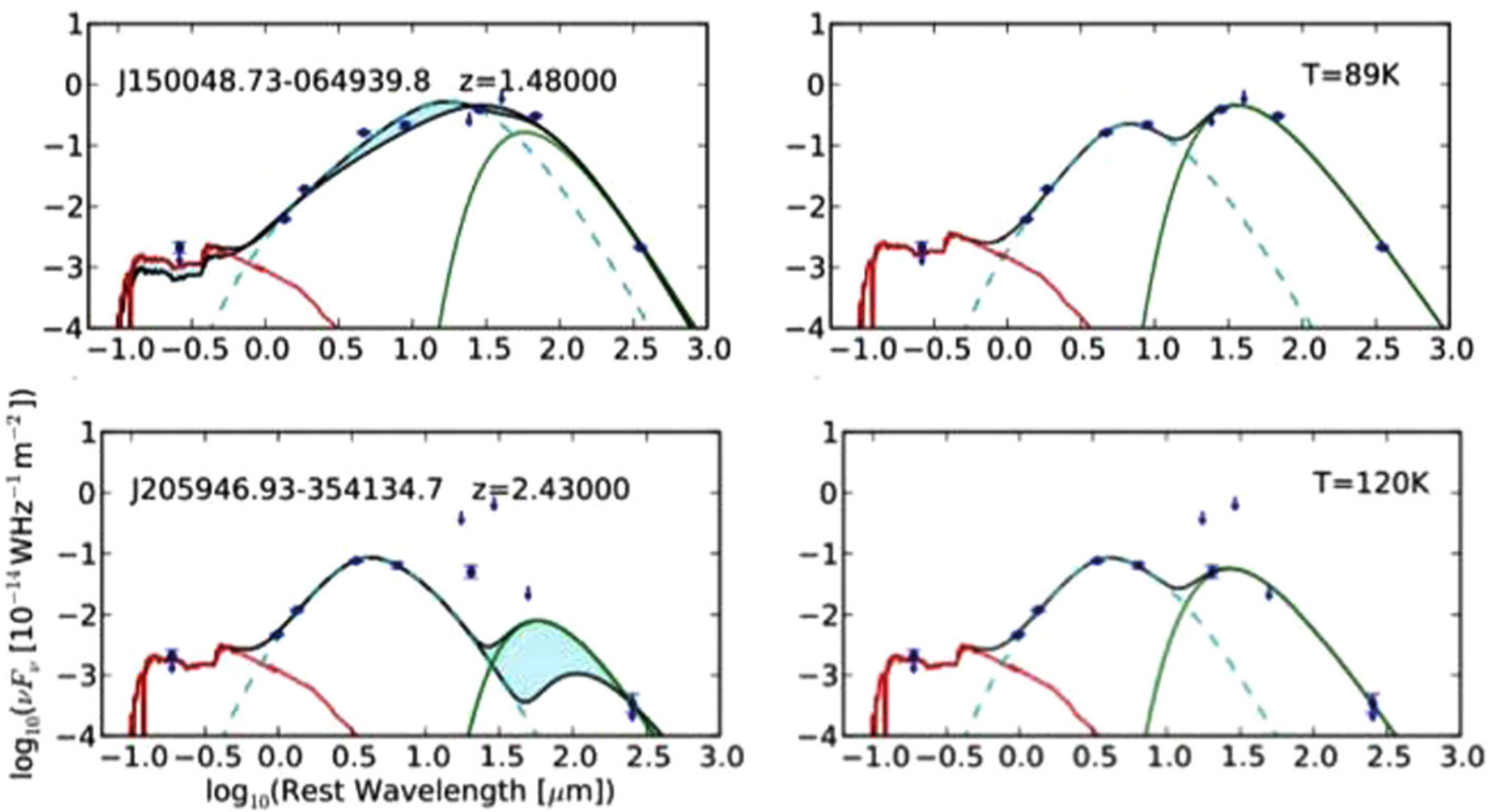

Figure 7. SED models for the two sources for which we have sufficient data near the peak of the SED, from Herschel, to obtain well constrained fits across the MIR and FIR: W1500-0649 and W2059-3541. "AGN" (dashed cyan line); modified blackbody "BB" dust component (green lines); stellar population (red lines); summed model (black line). For the AGN and stellar components, and for the total fits, only one fit is shown in each panel, for clarity. The ALMA data point is the rightmost point, and the limits near the peak of the cool component are 60 and $100 \mu \mathrm{m} I R A S$ data. Left: models with $T_{\mathrm{d}}=50 \mathrm{~K}, \beta=2$ (upper) and $T_{\mathrm{d}}=30 \mathrm{~K}, \beta=1.5$ (lower). Right: models in which the temperature of the BB dust component is allowed to float, for these two sources only, with $\beta=1.5$.

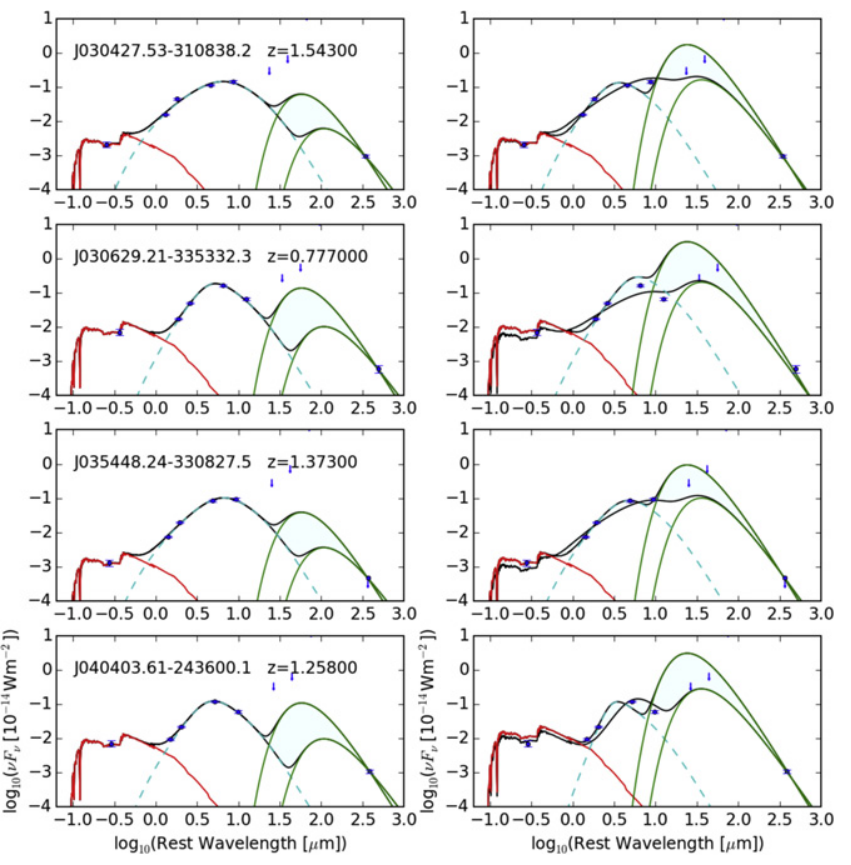

Figure 8. SED models for each source: data and fits as in Figure 7. Many of the BB models do not provide good fits, and these are not considered further in our analysis. In some cases no consistent fit is found for the stellar component. Left: models with $T_{\mathrm{d}}=50 \mathrm{~K}, \beta=2$ (upper) and $T_{\mathrm{d}}=30 \mathrm{~K}$, $\beta=1.5$ (lower). Right: models with $90 \mathrm{~K}$ (lower) and $120 \mathrm{~K}$ (upper), with $\beta=1.5$; temperature choices based on the results for W1500-0649 and W2059-3541 in Figure 7. depends strongly on the assumed rest-frame SED. In our situation this is particularly tricky because the unknown depth of the silicate feature will affect the observed $f_{22}$ flux density strongly at redshifts near 1.5 (e.g., see Figure 3). We have used the QSO2 ("Torus") template of Polletta et al. (2007) because it was successfully fitted by them (their Figure 9) to a very red, Compton-thick, Spitzer-selected obscured QSO that is similar in NIR-MIR spectral shape to our sources. To this we have grafted on the silicate absorption feature from the Arp 220 template of Polletta et al. (2007). We also derived the $k$ correction without the added silicate feature. All of the templates have been convolved with the WISE $22 \mu \mathrm{m}$ filter as a function of redshift by Polletta et al. (2007). Our quasars have comparable $k$-corrected $q_{22}$ values to the most radiopowerful and most radio-loud sources in the large Spitzer Subaru X-ray Deep Field sample of Ibar et al. (2008), and all of our sources would be considered to be moderately to very radio-loud after the $k$-correction, based on this criterion. This is the case even if the large silicate optical depth is omitted from the template. This conclusion is also apparent from Figure 4, in which our quasars lie between the radio-quiet (RQ) and radioloud (RL) quasar templates, when normalized at $4.6 \mu \mathrm{m}$ rest.

\subsection{BH Masses and Accretion Rates}

Lacking a high quality spectroscopic indicator of $\mathrm{BH}$ masses such as the $\mathrm{Mg}$ II emission line width, we derive $\mathrm{BH}$ masses and accretion rates from the AGN luminosities. These three parameters are therefore directly proportional to each other in 

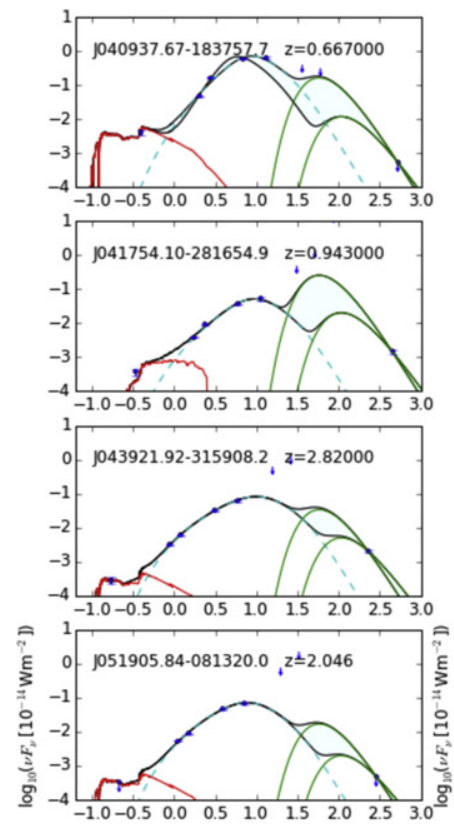

$\log _{10}($ Rest Wavelength $[\mu \mathrm{m}])$

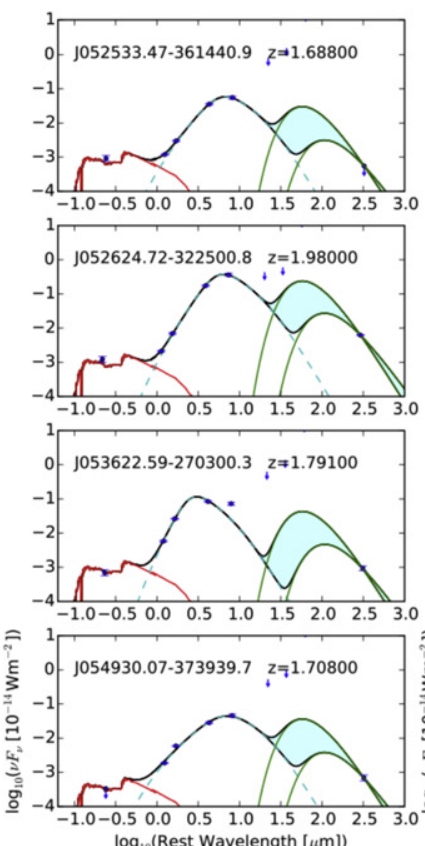

$\log _{10}($ Rest Wavelength $[\mu \mathrm{m}])$
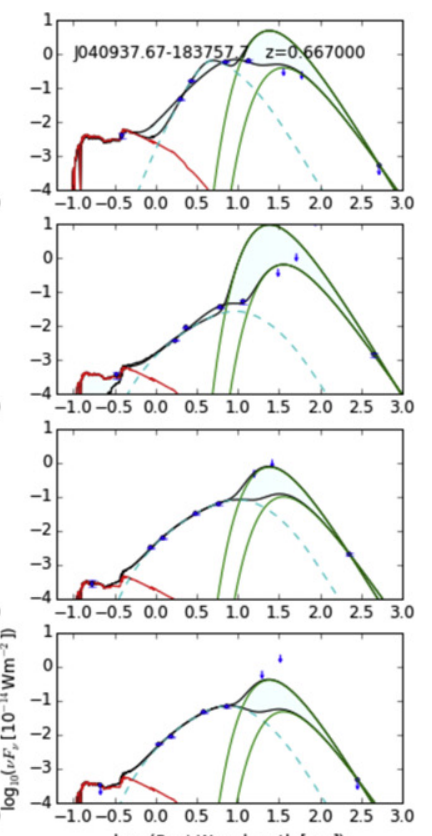

$\log _{10}($ Rest Wavelength $[\mu \mathrm{m}])$
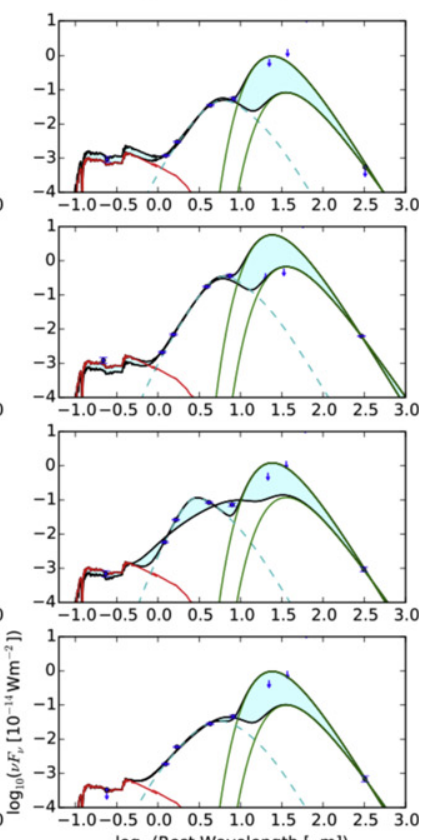

$\log _{10}($ Rest Wavelength $[\mu \mathrm{m}])$

Figure 8. (Continued.)

this work. We assume an Eddington ratio, $\lambda_{\text {Edd }}$, of 0.25, which is typical of $z \sim 2$ quasars (Kormendy \& Ho 2013):

$$
L_{\mathrm{Edd}}=\left(4 \pi G m_{p} c / \sigma_{\mathrm{T}}\right) M_{\mathrm{BH}}=3.3 \times 10^{4} M_{\mathrm{BH}} .
$$

We assume a covering factor of unity and spherical symmetry, consistent with late-stage mergers with heavily obscured nuclei. The covering factor cannot actually be this high in most sources, since emission lines from the NLR are visible in many cases; therefore our AGN luminosities and BH masses may be underestimates due to missing emission in the $\mathrm{X}$-ray to optical range which is not absorbed by the dust. We present the results in Table 5, columns 3-5, using our $L_{\mathrm{AGN}}$ Min,$L_{\mathrm{AGN}-\mathrm{Max}}$, and $L_{\text {Total-Best }}$ estimators. The median $/$ mean $\mathrm{BH}$ mass values are $1.0 / 1.15,1.07 / 1.45$, and $1.55 / 2.75 \times 10^{9} M_{\odot}$
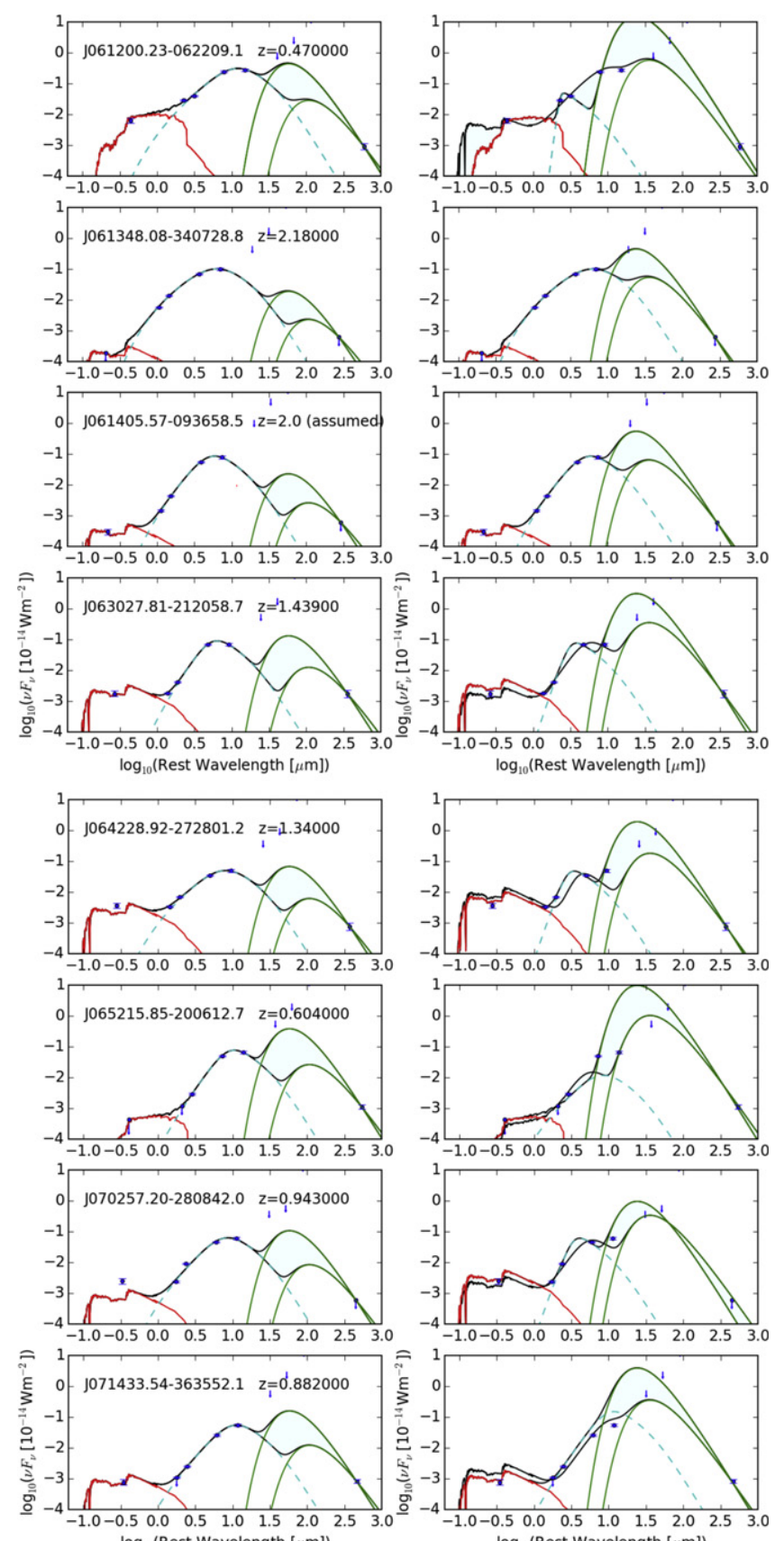

$\log _{10}($ Rest Wavelength $[\mu \mathrm{m}])$
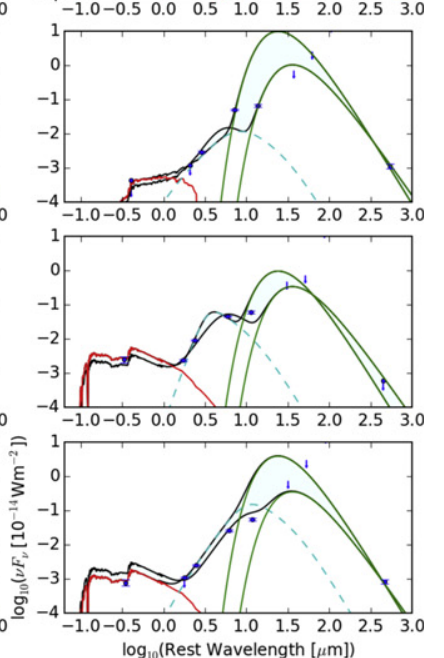

Figure 8. (Continued.)

respectively. In columns 6 and 7 we also list the BH masses derived by Kim et al. (2013), based on the [O III] $\lambda 5007 \AA$ line luminosity.

The accretion rates are derived from the AGN luminosities assuming an efficiency for the conversion of matter into radiant energy of $\epsilon=0.1$ (Heckman \& Best 2014):

$$
L_{\mathrm{bol}}=\epsilon \dot{M} c^{2} \text {. }
$$

The rates are listed in columns $8-10$ of Table 5 , and range from 0.24 to $25.3 M_{\odot} \mathrm{yr}^{-1}$, with median/mean values of $6.1 /$ 5.6, 7.6/6.2, and $14.5 / 9.1 M_{\odot} \mathrm{yr}^{-1}$ respectively for the AGNMin, AGN-Max, and Total-Best values. The overall ranges in BH mass and accretion rate are listed in Table 4. 

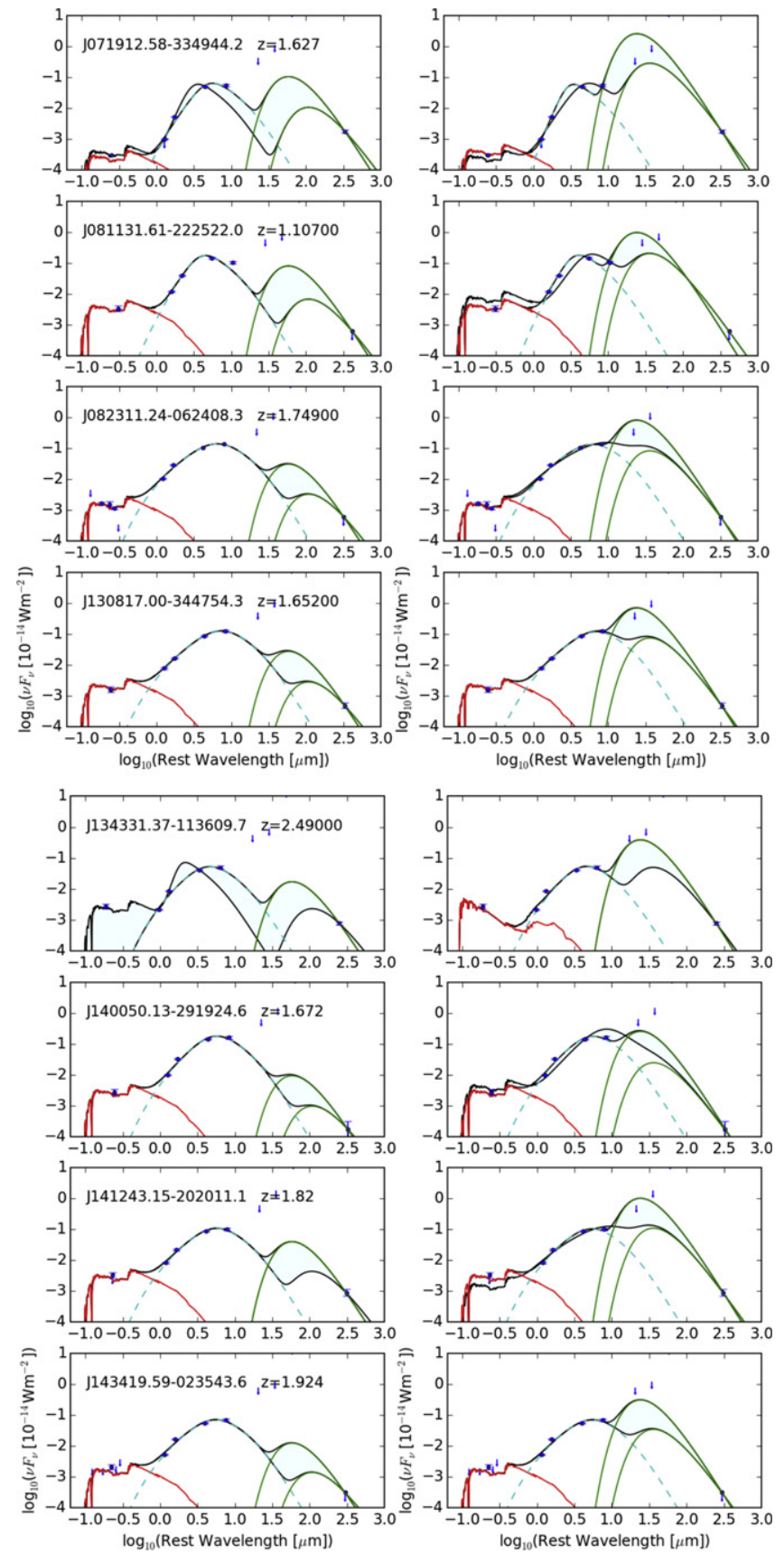

Figure 8. (Continued.)

\section{DISCUSSION}

Our goals are to search for the most luminous obscured quasars at redshifts $\sim 1-3$, the peak epoch of massive $\mathrm{BH}$ building, which are in the process of quenching star formation, and to investigate the role of radio jets in that process. We are specifically interested in the kinetic role of moderate to high power jets on the ISM within the galaxy host, while the AGN is still accreting strongly in "quasar-mode." This is in contrast to the role of jets in typical "radio-mode" AGNs, which are thought to be accreting at low rates and to have a role in maintaining galaxies free of infalling gas. As outlined in Section 1, models that take the porosity of the ISM into account show that high power jets could be quite effective in a dense
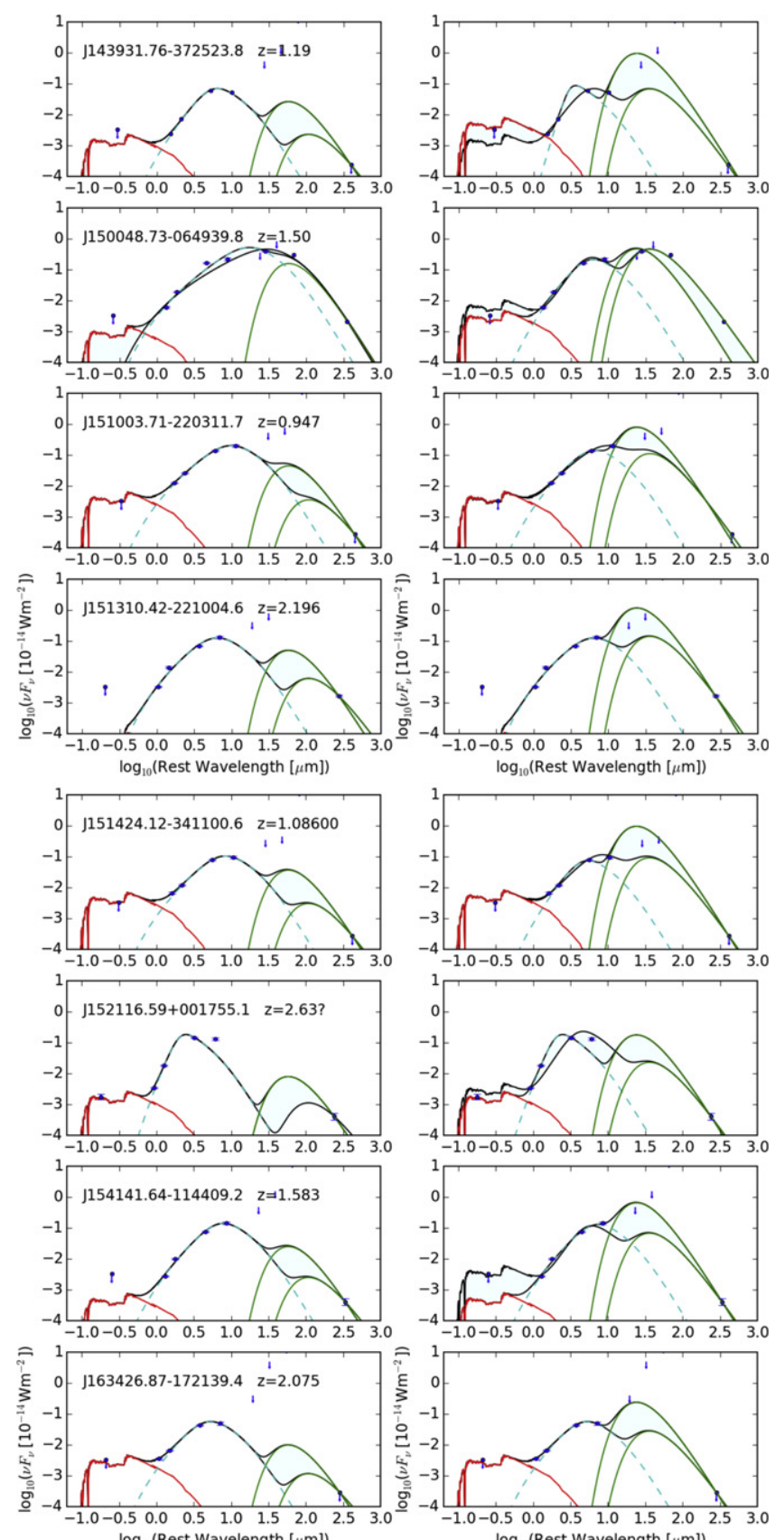

Figure 8. (Continued.)

dusty environment, such as found in the central regions of major mergers (Wagner \& Bicknell 2011; Wagner et al. 2012).

We have shown that WISE has found sources that are steeper (redder) in the rest-frame $1-10 \mu \mathrm{m}$ range than most known Spitzer-selected red sources, including the so-called DOGs. The two other samples of very red WISE sources, the Hot DOGs and the WLABs (EWB12) that were selected without regard for radio emission, have extremely red rest-frame 1-10 $\mu \mathrm{m}$ SEDs which are very similar to those of our radioselected sample. The radio-blind samples have higher average redshifts than our radio-powerful sample, probably as a result of the different WISE-optical color selection criteria. We attribute our ability to identify these extremely red sources to the much larger survey volume of WISE compared to Spitzer. 

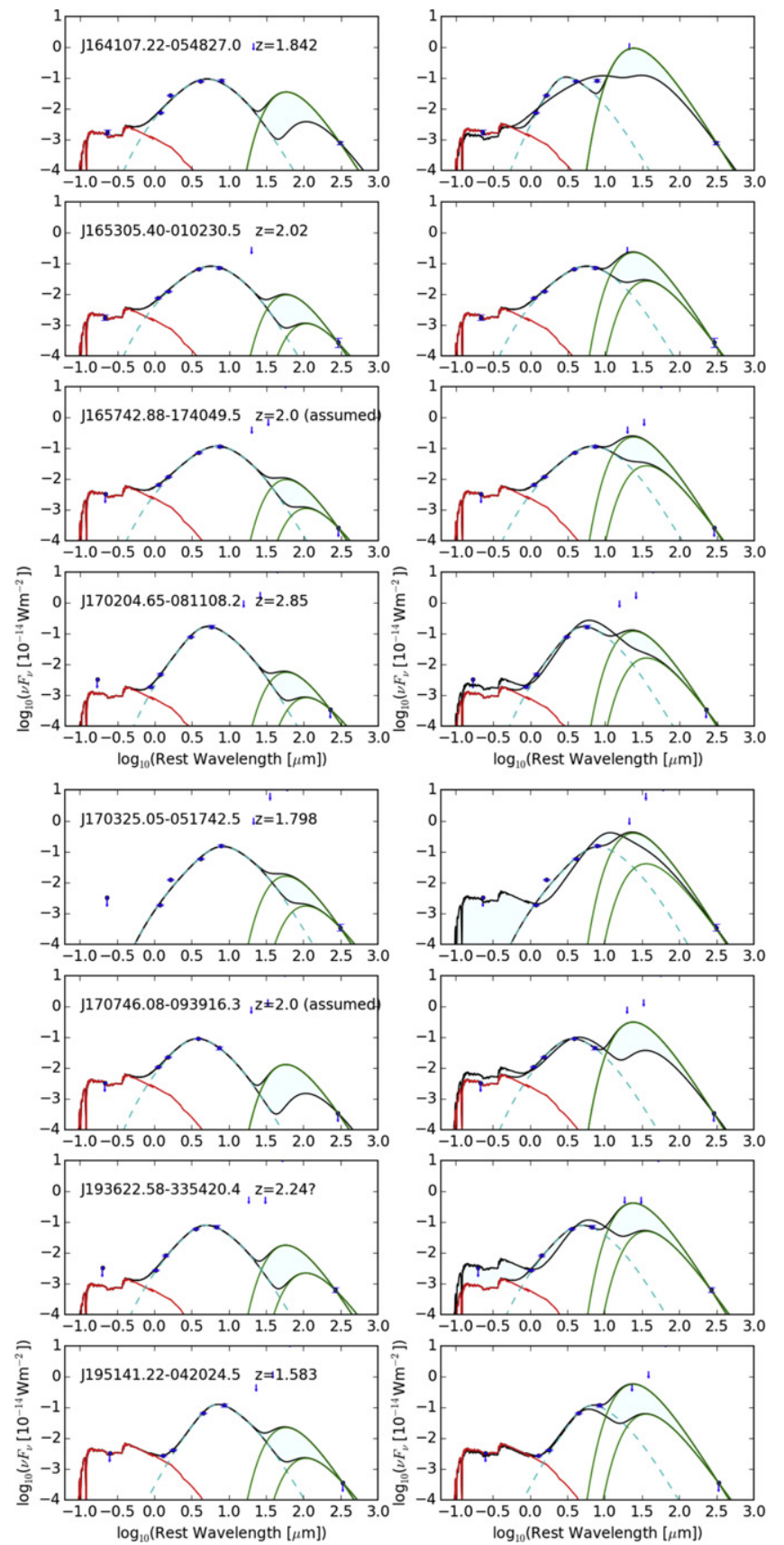

Figure 8. (Continued.)

These systems are candidate luminous quasar-mode AGNs that are highly obscured, and the radio-bright ones selected here potentially have young jet activity. Recent X-ray observations of a few Hot DOGs confirm the likelihood of highly buried AGNs (Stern et al. 2014; Pinconcelli et al. 2015). The hydrogen column for $\mathrm{W} 1835+4355$ was found to be $N_{\mathrm{H}} \geqslant 10^{23} \mathrm{~cm}^{-2}$ by Pinconcelli et al. (2015).

We also find that the WISE-NVSS-ALMA sample sources are more strongly dominated by AGNs than the Spitzer DOGs. SFRs of hundreds up to a few thousand $M_{\odot} \mathrm{yr}^{-1}$ could also be present in some of the systems, although the IR-submillimeter SEDs of some of the ALMA sources, in particular those with only upper limits at $345 \mathrm{GHz}$, may be consistent with the AGN torus model in Figure 4 without any SF contribution. Therefore
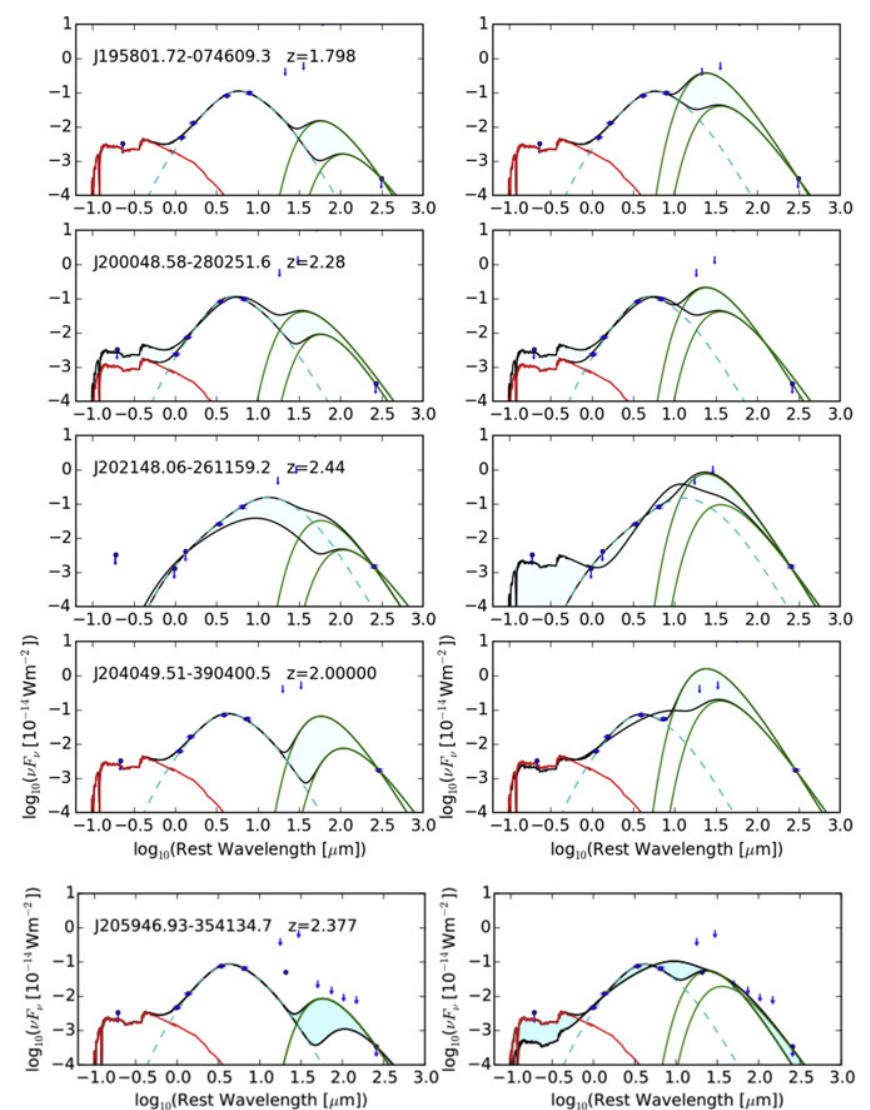

Figure 8. (Continued.)

these sources are indeed objects in which it is likely that the accretion rates are still very high but the SFRs are low relative to the accretion power, and could thus be ideal sources for investigating recent and ongoing quenching by jet-powered AGN feedback.

Some of our systems could be HERGs seen at high inclination through an optically thick torus, and it is possible that we have included some with lobes that are unresolved by the $45^{\prime \prime}$ NVSS beam. We will show in the next paper (Carol J. Lonsdale et al. 2015, in preparation) that our $8-12 \mathrm{GHz}$ VLA data rule out this scenario for most sources, although a small subset of the VLA sample of 156 red obscured quasars does indeed turn out to have large (several Mpc scale) double lobes $(\sim 7 \%)$. Another small percentage shows evidence of smallscale double lobes on scales $\sim 2-10 \mathrm{kpc}$.

\subsection{The AGN-heated Source}

In this work we have derived a range in the plausible AGN luminosities depending on whether we assume that any of the far-infrared luminosity component is AGN-heated:

$$
L_{\mathrm{bol}, \mathrm{AGN}}=L_{\mathrm{AGN}, \mathrm{MIR}}+f_{\mathrm{AGN}} L_{\mathrm{BB}}
$$

where $L_{\mathrm{bol}, \mathrm{AGN}}$ is the bolometric luminosity of the AGN, $L_{\mathrm{AGN}}$, MIR is the MIR AGN luminosity from the fit to Equation (2), $f_{\mathrm{AGN}}$ is the fraction of the far-infrared emission contributed by the AGNs and $L_{\mathrm{BB}}$ is the far-infrared luminosity. $f_{\mathrm{AGN}}$ is assumed to be 0 for the $L_{\mathrm{AGN}}$ models and to be 1 for the scenario in which we assume that $L_{\text {Total-Best }}$ is completely AGN-powered. The warm AGN component luminosity is very 
insensitive to the dust temperature of the $\mathrm{BB}$ component, due to the strong constraints placed on the AGN model shape by the four WISE data points. The range of values for the ratio of the maximum plausible AGN luminosity to the minimum estimate is $1.1<L_{\text {Total-Best }} / L_{\mathrm{AGN}-\text { Min }}<5.5$, with a median value of 2.0 .

We have assumed a covering factor of warm $(\sim 300 \mathrm{~K})$ dust that emits in the MIR, $\Omega_{\mathrm{WD}}$ :

$$
L_{\mathrm{bol}, \mathrm{AGN}}=L_{\mathrm{AGN}, \mathrm{MIR}} / \Omega_{\mathrm{WD}}
$$

of unity and spherical symmetry, consistent with late-stage mergers with heavily obscured nuclei. This may not be a valid assumption, in which case we may have underestimated the AGN luminosities and $\mathrm{BH}$ masses. In particular, narrow emission lines photoionized by the AGNs are seen in many cases, therefore these luminosity estimators may be excluding flux emitted by the AGNs that does not intercept the dusty structure. A further complication is that the covering factor of the cold $(\sim 50 \mathrm{~K})$ dust is likely to be different from that of the hot dust, and its optical depth to the MIR emission is unknown.

An added complication is the possibility that a non-spherical source emits non-isotropically because it is optically thick, as is often the case for torus models (e.g., Efstathiou et al. 2013). In that case a further correction for anisotropy is required, and this depends on the particular torus model and also on the inclination of the torus. Generally speaking, for edge-on viewing the luminosity will be underestimated whereas for face-on viewing it may be overestimated. Face-on viewing is ruled out by the red MIR-optical SEDs and therefore we can conclude that $L_{\mathrm{AGN}, \mathrm{MIR}}$ provides a firm lower limit to the bolometric luminosity of the AGNs.

Support for the high covering factor interpretation for most of our WISE-NVSS-ALMA sample comes from the overall relative numbers of obscured and unobscured AGNs among the highest luminosity radiative $=$ mode $\mathrm{AGNs}$ at high redshift $(z \sim 1-3)$, which are roughly equal (Assef et al. 2015; Lacy et al. 2015; Tsai et al. 2015). Taken at face value this would imply an average covering fraction of $\sim 50 \%$, if these populations differ only by orientation (ignoring the differing selection functions for type 1 and type 2 AGNs, Elitzur 2012).

\subsubsection{A Torus?}

Although we have commented above on the likelihood that the covering factor may be lower than 1.0 for many sources, it might be questionable to conclude that a classical smooth torus could be responsible for the very high luminosities found for some sources, because they would require very large tori (several hundred pc to over a $\mathrm{kpc}$ in diameter), especially if the $L_{\text {Total-Best }}$ values are interpreted as fully AGN-powered. Such large thin structures would be unstable. A clumpy torus would be more plausible, as it could achieve a wider range of dust temperatures than a smooth torus of the same diameter.

A torus-like structure would imply that a large fraction of the total AGN luminosity escapes dust absorption and would be easily visible at optical wavelengths. Since the line-of-sight optical continuum emission is very faint compared to type 1 AGNs (Figure 4) the tori would all have to be inclined closely to our line of sight. Our selection function of course has favored the selection of highly obscured systems, and we do indeed find a fraction of our sample to have (small) double radio lobes in the plane of the sky (Section 5.4), as might be expected for a radio quasar or radio galaxy interpreted as viewed through an edge-on disk in the standard unification picture. The majority of our sample does not display extended radio lobes, however.

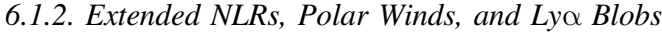

High-resolution observations of nearby low-luminosity AGNs show that the MIR emission can lie in the polar direction and may be associated with NLR clouds or with thermal winds from the AGNs (Zhang et al. 2013 and references therein). It is also known that a significant fraction of FRII radio AGNs have extended emission line regions (EELRs) up to tens of kpc in size (Fu \& Stockton 2007), which are outflowing (Shih \& Stockton 2014). The CSS sources, which are younger versions of the FRIIs, have smaller EELRs that are better aligned with the radio structures than they are in FRIIs (Axon et al. 2000). Therefore it is possible that some of the MIR emission in our sample is associated with NLR or EELR clouds that are heated by the central AGN, or shockheated by the radio jet interactions (Mullaney et al. 2013). Efstathiou et al. (2013) have found that a dusty NLR component is needed to fit the MIR-FIR SED of the hyperluminous galaxy IRAS10214 +4724 at $z=2.285$.

Bridge et al. (2013) have discovered that a subset of the Hot DOGs that have extended faint optical emission also possess Lyman alpha Blobs (LABs; defined to have Ly $\alpha$ emission extended on scales $>30 \mathrm{kpc}$ ). The WISE LABs are nonsymmetric and there is evidence for large outflow velocities. The presence of extended ionized gas suggests significant shock heating or that a significant fraction of the nuclear ionizing radiation must be able to find its way out of the galaxy, as in the radio galaxy EELRs, even in these highly obscured MIR-dominated systems. Few of our sources currently have spectra that cover Ly $\alpha$. W0613-3407 has Ly $\alpha$ extended spatially on a scale of $3^{\prime \prime}$, so qualifies as an LAB by the size definition. W1343-1136 appears to also have extended emission (about 2 ". $5 ; 25 \mathrm{kpc}$ ) but to just miss the usual LAB definition of $>30 \mathrm{kpc}$. Although it remains to be seen whether a large fraction of our WISE-NVSS-ALMA sources possess LABs, we have some evidence that some of them possess broad forbidden emission lines that might indicate substantial outflows. Kim et al. (2013) find that the [O III] lines are exceptionally broad for six of our quasars, with full width at half maximum $\sim 1300-2100 \mathrm{~km} \mathrm{~s}^{-1}$, significantly larger than that of typical distant quasars.

\subsection{Star Formation}

The contribution to the $1-1000 \mu \mathrm{m}$ luminosity from star formation has larger overall uncertainty than the AGN contribution because star formation can produce a larger range in observed dust temperature, varying by $\sim 2$ dex between the viable 30 to $120 \mathrm{~K} \mathrm{BB}$ models (Table 6, columns 4-6). The maximum star formation luminosity, $L_{\mathrm{BB}-\mathrm{Max}}$, is comparable to the the AGN luminosity estimators, while the minimum starburst luminosity, coming from the $30 \mathrm{~K}$ model in all but one case, can be well over an order of magnitude smaller than the AGN luminosity. A minimum SFR of 0 is also possible if $L_{\text {Total-Best }}$ is interpreted as completely AGN-powered.

If we adopt $50 \mathrm{~K}$ dust for the $\mathrm{BB}$ component, the SFRs lie between $\sim 200$ and $3500 M_{\odot} \mathrm{yr}^{-1}$. The lower values are consistent with main-sequence galaxies at these redshifts while 


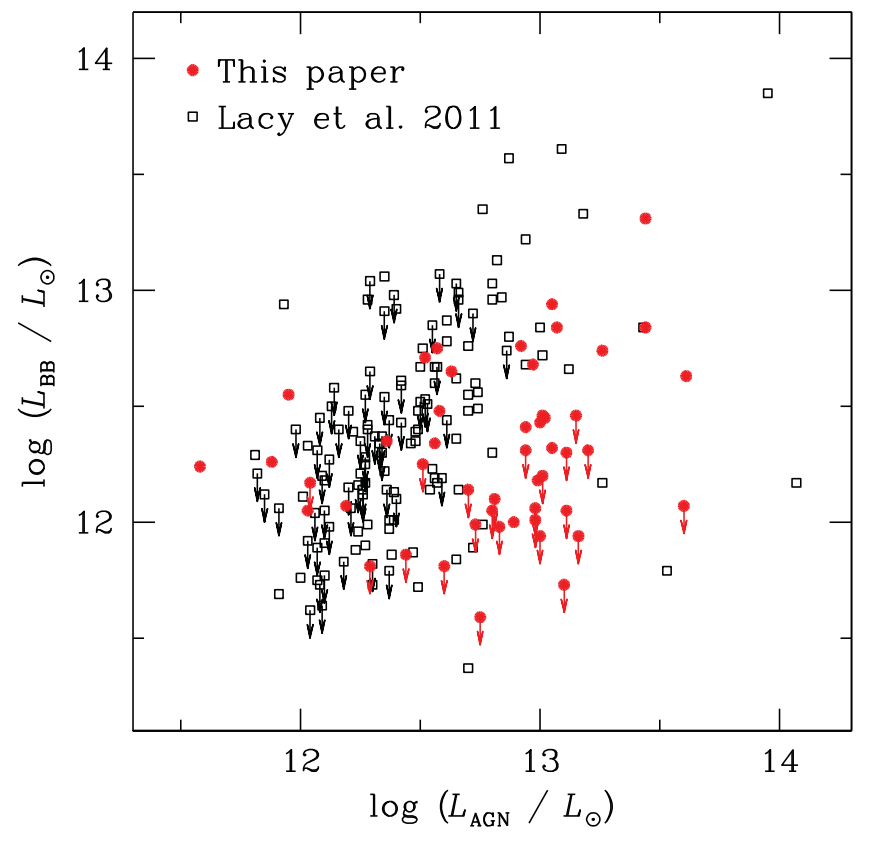

Figure 9. Comparison of the Spitzer-selected sample of Lacy11 with our sample in the $L_{\mathrm{AGN}}$ vs. $L_{\mathrm{BB}}$ plane. For this figure we show the BB luminosity from our $50 \mathrm{~K}$ model to match the method used by Lacy 11.

the higher values may require a starburst (Delvecchio et al. 2015).

Our RT modeling of our best observed source favored the presence of a young (10-15 Myr), presumably compact, starburst contributing about $50 \%$ of the $1-1000 \mu \mathrm{m}$ luminosity. Compact starbursts have been investigated in several local galaxies. The nuclear structures around the twin nuclei of Arp 220 are thought to be powered by compact starbursts with relatively high dust temperatures (over $100 \mathrm{~K}$ ) and to be highly optically thick out to $\gg 100 \mu \mathrm{m}$ (Wilson et al. 2014; BarcosMunoz et al. 2015; Scoville et al. 2015). Tsai et al. (2015) have considered in more detail the possibility that a compact starburst could contribute a significant luminosity to their most luminous Hot DOGs, those with $L_{\text {bol }}>10^{14} L_{\odot}$. Using He 2-10 as a local analog and the STARBURST99 code (Leitherer $\&$ Chen 2010), they find SFRs $>5 \times 10^{3} M_{\odot} \mathrm{yr}^{-1}$ for the conservative case of a top heavy IMF.

Tsai et al. (2015) conclude that there is insufficient $\mathrm{CO}$ in these systems to support very large amounts of star formation. In our case we find mean ISM masses of between $\sim 2$ and $9 \times$ $10^{10} M_{\odot}$, depending on the assumed dust temperature. For the range of estimated SFRs across our models the gas would be depleted in $\sim 2 \mathrm{Myr}-2 \mathrm{Gyr}$. The lower depletion times (corresponding to the higher SFRs) are not insupportable, in the scenario of a late-stage, violent, gas-rich merger. Therefore it is quite possible that a vigorous starburst is present in some of our systems, and that a compact young starburst may contribute to the warmest dust emission.

\subsection{High Accretion Rates Relative to Star Formation}

In Figure 9 we plot $L_{\mathrm{AGN}-M i n}$ versus $L_{\mathrm{BB}-50 \mathrm{~K}}$ : no correlation is apparent. We compare our sample to the radio-quiet SpitzerIRAC-selected quasar sample of Lacy et al. (2011, Lacy11), for which fits have been done using the same formalism as here. The Lacy11 sample has a wider range in mid-IR color selection than our sample, including Type 1 quasars, reddened Type 1 quasars and Type 2 quasars, and is therefore representative of the IR-bright AGN population as a whole. There is some evidence that the heavily obscured quasars in our sample have similar far-infrared luminosities to the Lacy11 sample, i.e., similar SFRs, but systematically higher AGN luminosity. This is consistent with them being in a phase of systematically higher accretion rate relative to star formation.

\subsection{ISM Masses}

The masses of the BH, the ISM, and the stellar component are tabulated in Table 5. The ISM mass is directly proportional to the $870 \mu \mathrm{m}$ luminosity, but depends much less strongly on dust temperature than do $L_{\mathrm{BB}}$ and SFR.

We found in Section 5.1 that the ISM masses are comparable to those of the "IR-bright" high-redshift sources in the COSMOS field (Scoville et al. 2014). We can also compare our sample to the compilation of all known molecular masses for $z>1$ systems of Carilii \& Walter (2013), their Figure 9. Assuming a $\mathrm{CO}$ to $\mathrm{H}_{2}$ conversion factor appropriate for starbursts (Downes \& Solomon 1998; Bolatto et al. 2013) of $\alpha_{\mathrm{CO}} \sim 0.8 M_{\odot} /\left(\mathrm{K} \mathrm{km} \mathrm{s}^{-1} \mathrm{pc}^{2}\right)$, and assuming $50 \%$ of the gaseous ISM is in molecular form (Carilii \& Walter 2013), our median detected ISM mass implies a median molecular gas mass of $2.95 \times 10^{10} M_{\odot}$ and a median CO line luminosity of $3.7 \times 10^{10} \mathrm{~K} \mathrm{~km} \mathrm{~s}^{-1} \mathrm{pc}^{2}$. This lies at about the 80th percentile of the high-redshift QSOs in Figure 9 of Carilii \& Walter (2013), and about the 50th percentile of the SMGs. It is lower than four of the RGs in this figure and comparable to the other two.

In summary, if we assume no contribution to the measured ALMA fluxes from non-thermal synchrotron emission, we find that the implied ISM masses are quite high, comparable to those of typical "IR-bright" star-forming systems in the COSMOS field at these redshifts, to the most gas-rich galaxies in the local CO survey of Leroy et al. (2009), and consistent with the large $\mathrm{CO}$ masses of $z>1$ QSOs, SMGs, and RGs. This gas and dust could exist in nuclear, AGN-heated structures, or it could be powered by star formation somewhere within the host system.

\subsection{BH Masses}

The BH mass estimates derived by Kim et al. (2013) for three of the six quasars with available [O III] $\lambda 5007 \AA$ line luminosities are significantly larger than the values derived from our MIR data. Our masses may be underestimated due to extreme extinction in the MIR (although we might expect to recover such dust-absorbed energy in the FIR-submillimeter), and our assumption of $\sim 100 \%$ covering factor may be incorrect. Another possibility is that the [O III] line strengths of Kim et al. (2013) are boosted by shocks and outflows. We will address the relationship between $L_{\mathrm{MIR}}$ and $L_{[\mathrm{O} \text { II] }}$ for these systems (cf. Mullaney et al. 2013) in a forthcoming paper presenting the spectroscopy.

\subsection{Nature of the Radio Sources}

We will address the morphology of the radio sources in our paper presenting the VLA results, where we will show that the majority of the sample are compact on $\sim 1-3 \mathrm{kpc}$ scales. The rest-frame $3 \mathrm{GHz}$ radio powers lie in the range log $\left(P_{3.0} \mathrm{GHz}\right.$ $\left./ \mathrm{W} \mathrm{Hz}^{-1}\right)=24.74-27.33$. The radio power of both RQ and RL systems evolves with redshift (Best et al. 2014), and some 

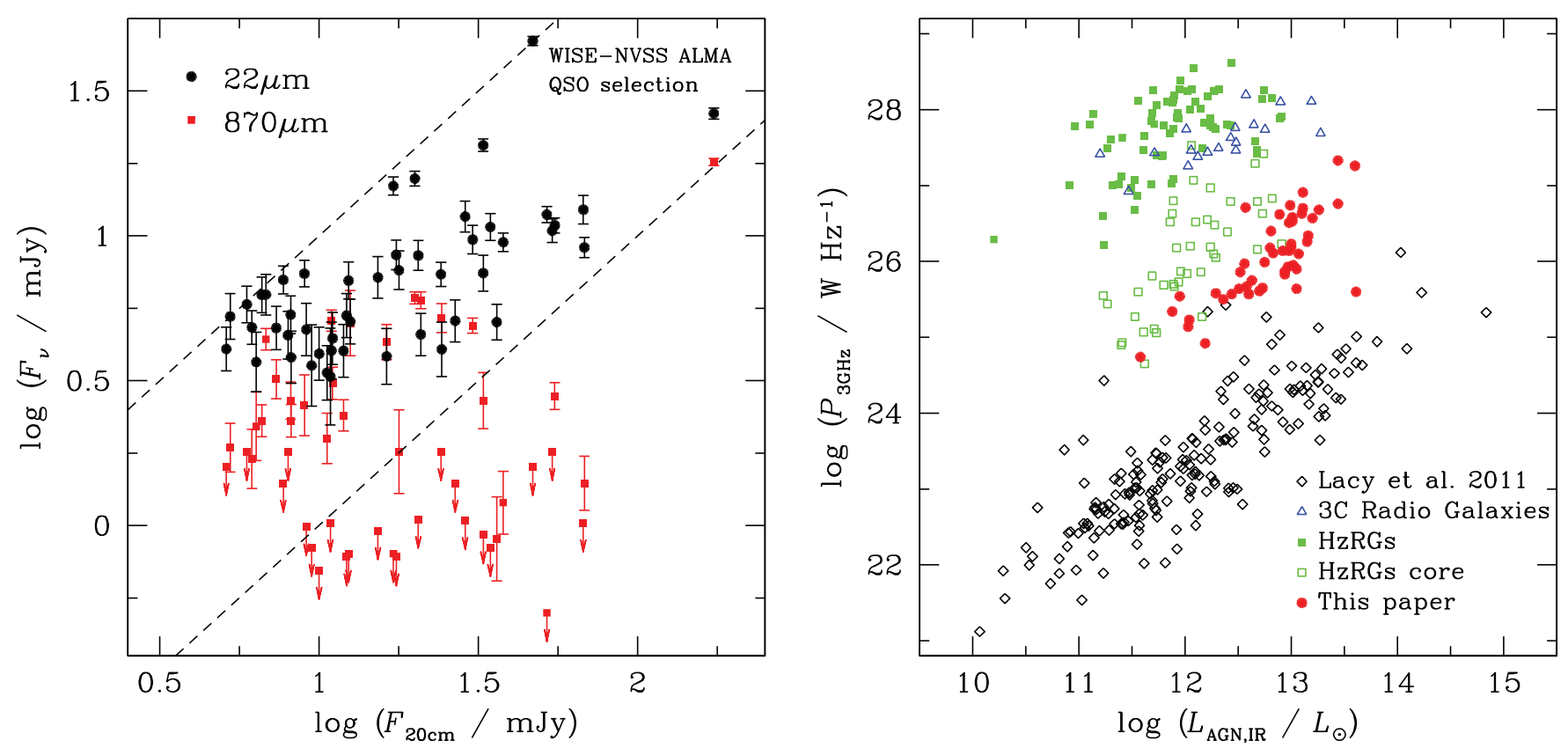

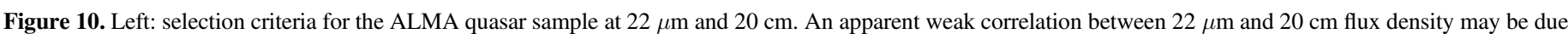

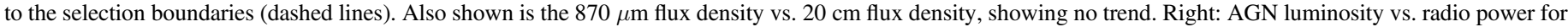

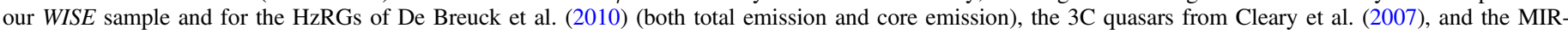
selected AGNs of Lacy11.

radio sources at $z=1-3$ with radio powers in this range are found to be radio-quiet based on the $24 \mu \mathrm{m} q$ value, $q_{24}=\log \left(f_{24 \mu \mathrm{m}} / f_{20 \mathrm{~cm}}\right)$ (Simpson et al. 2012). Our sources have values of $q_{22}$ that are significantly too low for them to be considered radio-quiet by this criterion.

In Figure 10 -right we compare $L_{\mathrm{AGN}-M i n}$ with $P_{\text {Radio-3 GHz }}$, and also include the high-redshift $(1<z<5.2)$ radio galaxy (HzRG) sample of De Breuck et al. (2010) and the 3C radio galaxy sample of Cleary et al. (2007) at $0.4<z<1.2$. For the HzRG sample we also include the estimated core flux, using the $20 \mathrm{GHz}$ core fractions given by De Breuck et al. (2010) and assuming that the same fraction is appropriate at $3 \mathrm{GHz}$. There is a large disparity between our sample, the Lacy11 sample, and the two high-redshift samples. The HzRGs and 3C RGs have 2-3 orders of magnitude more radio power than our sample, for a given mid-IR AGN luminosity, which is expected given that the power of the HzRGs is dominated by the extended lobes. The core radio powers for the classical HzRGs have a similar range to our sample but our sources are significantly more luminous in the infrared, consistent with a higher accretion rate. The radio-blind MIR-selected sample of Lacy11 has on average 2-3 orders of magnitude less radio power than our sample, as expected for an RQ-dominated sample.

All four samples in this figure display an apparent correlation between $L_{\mathrm{AGN}}$ and $P_{\text {Radio-3 GHz}}$. Given the rarity of these sources and the large redshift range involved, the correlation for our sample may be an artifact of Malmquist bias resulting from the $22 \mu \mathrm{m}$ and the $1.4 \mathrm{GHz}$ flux density thresholds. We show in Figure 10-left that there exists an apparent correlation between $22 \mu \mathrm{m}$ and $20 \mathrm{~cm}$ flux density that may be caused by the limited dynamic range in the flux ratio selection thresholds for the WISE-NVSS-ALMA sample: $-1<\log \left(f_{22 \mu \mathrm{m}} / f_{20 \mathrm{~cm}}\right)<0$ (dashed lines). Therefore the apparent correlation seen between
$L_{\mathrm{AGN}}$ and $P_{\text {Radio-3 GHz }}$ in Figure 10-right may not be real, at least for our sample.

\section{CONCLUSIONS}

We have selected a sample of extremely red, luminous, RP sources in the $0.5<z<3$ redshift range using WISE MIR colors and the NVSS and FIRST $20 \mathrm{~cm}$ radio surveys. We present ALMA $870 \mu \mathrm{m}$ photometry for 49 southern sources from the total sample of 156 red sources, and redshifts for 45 of them from a combination of optical and near-infrared spectroscopy. JCMT imaging at $850 \mu \mathrm{m}$ has been presented for 30 of the northern sample by Jones et al. (2015). Combined, 30 sources have a detection at 850 or $870 \mu \mathrm{m}, 25$ of them with a known redshift. We also have $R$-band imaging for 27 sources, Herschel photometry for 15 sources, including two of the ALMA sample, and CSO $350 \mu \mathrm{m}$ data four additional sources, including two from the ALMA sample.

Having compared the SEDs of the red WISE-selected sources with other samples and template SEDs, we conclude that the rest-frame MIR-submillimeter SEDs of our WISE-NVSS sources are dominated by AGN emission in the MIR. They have extremely red optical-MIR colors and high bolometric luminosities in the ULIRG and HyLIRG regime, in some cases approaching or exceeding $10^{14} L_{\odot}$. They are redder in the NIRMIR than previous samples selected from Spitzer surveys, including almost all of the Spitzer DOGs. They also display systematically warmer overall MIR-submillimeter SEDs, and probably have higher levels of accretion, relative to star formation, than the DOGs. BH mass estimates for our sample are $7.7<\log \left(M_{\mathrm{BH}} / M_{\odot}\right)<10.2$. We conclude that these sources are best labeled as obscured RP QSOs. The rest-frame $3 \mathrm{GHz}$ radio powers are $24.7<\log \left(P_{3.0 \mathrm{GHz}} / \mathrm{W} \mathrm{Hz}^{-1}\right)<27.3$, and all sources are radio-intermediate or radio-loud. The ability of WISE to find this rare and unique sample is due to the large volume accessible to WISE. 
Our best constrained source has RT solutions with approximately equal contributions for an obscured AGN and a young (10-15 Myr) compact starburst. Simpler two-component fits to the whole sample find that the SFRs of the sample could be in the range of hundreds to thousands of solar masses per year, but it also possible to fit a significant fraction of the ALMA data for some sources with a centrally heated dusty structure, in which case most of the entire bolometric luminosity could be attributed to the obscured AGN. In that scenario it is likely that the emission is dominated by a small, high covering factor cocoon and/or an extended NLR.

Our sample is similar in MIR selection method to Eisenhardt et al. (2012), Wu et al. (2012), and Bridge et al. (2013), who did not use radio flux density as a selection criterion. Their Hot DOG samples exhibit similar SED shapes to our radio-selected sample. The MIR SEDs of the EWB12 samples may be steeper on average than those of our radio-selected sample, but this may be due to different selection effects between the MIRoptical color selection criteria for the two samples, resulting in an average higher redshift for the radio-blind sample.

This paper makes use of the following ALMA data: ADS/ JAO.ALMA\#2011.0.00397.S. ALMA is a partnership of ESO (representing its member states), NSF (USA), and NINS (Japan), together with NRC (Canada), NSC and ASIAA (Taiwan), and KASI (Republic of Korea), in cooperation with the Republic of Chile. The Joint ALMA Observatory is operated by ESO, AUI/NRAO, and NAOJ. This publication makes use of data products from the Wide-field Infrared Survey Explorer, which is a joint project of the University of California, Los Angeles, and the Jet Propulsion Laboratory/ California Institute of Technology, funded by the National Aeronautics and Space Administration. This work is based on observations made with the Caltech Submillimeter Observatory, which is operated by the California Institute of Technology under funding from the National Science Foundation, contract AST 90-15755. This paper uses data from SDSS (DR 8). Funding for SDSS-III has been provided by the Alfred P. Sloan Foundation, the Participating Institutions, the National Science Foundation, and the U.S. Department of Energy Office of Science. The SDSS-III web site is http://www.sdss3.org/. RJA was supported by Gemini-CONICYT grant number 32120009. We thank the anonymous referee for comments that helped improve the paper. The National Radio Astronomy Observatory is a facility of the National Science Foundation operated under cooperative agreement by Associated Universities, Inc.

Facilities: WISE, VLA, ALMA, CTIO (SOAR-Goodman), Palomar 200 inch, VLT, Herschel, CSO, Magellan.

\section{REFERENCES}

Aalto, S., Garcia-Burillo, S., Muller, S., et al. 2015, A\&A, 574, 85 Appleton, P. N., Fadda, D. T., Marleau, F. R., et al. 2004, ApJS, 154, 147 Appleton, P. N., Xu, K., Reach, W., et al. 2006, ApJS, 639, L51 Archibald, E. N., Dunlop, J. S., Hughes, D. H., et al. 2001, MNRAS, 323, 417 Ashby, M. L. N., Stern, D., Brodwin, M., et al. 2009, ApJ, 701, 428 Ashby, R. F., Stanford, S. A., Brodwin, M., et al. 2013, ApJS, 209, 22 Assef, R., Stern, D., Kochanek, C. S., et al. 2013, ApJ, 772, 26 Assef, R. J., Eisenhardt, P. R. M., Stern, D., et al. 2015, ApJ, 804, 27 Assef, R. J., Kochanek, C. S., Brodwin, M., et al. 2010, ApJ, 713, 970 Axon, D. J., Robinson, A., Young, S., Smith, J. E., \& Hough, J. H. 2000, AJ, 120,2284

Baldwin, J. A., Phillips, M. M., \& Terlevich, R. 1981, PASP, 93, 5 Banerji, M., Fabian, A., \& McMahon, R. G. 2014, MNRAS, 439, L51
Barcos-Munoz, L., Leroy, A. J., Evans, A. S., et al. 2015, ApJ, 799, 10 Becker, R. H., White, R. L., \& Helfand, D. J. 1995, ApJ, 450, 559 Bendo, G., Baes, M., Biancji, S., et al. 2015, MNRAS, 448, 135 Best, P. N., \& Heckman, T. M. 2012, MNRAS, 421, 1569

Best, P. N., Ker, L. M., Simpson, C., Rigy, E. E., \& Sabater, J. 2014, MNRAS, 445,955

Bolatto, A. D., Wolfire, M., \& Leroy, A. K. 2013, ARA\&A, 51, 207

Bridge, C. R., Blain, A. W., Borys, C. J. K., et al. 2013, ApJ, 769, 91 (EWB12)

Carilii, C., \& Walter, F. 2013, ARA\&A, 51, 105

Cattaneo, A., \& Teyssier, R. 2007, MNRAS, 376, 1547

Cleary, K., Lawrence, C. R., Marshall, J. A., Hao, L., \& Meier, D. 2007, ASPC, 373, 475

Combes, F., Garcia-Burillo, S., Casaola, V., et al. 2013, A\&A, 558, 124

Condon, J. J., Cotton, W. D., Greisen, E. W., et al. 1998, AJ, 115, 1693

Croton, C. 2006, MNRAS, 365, 11

Cutri, R., Wright, E. L., Conrow, T., et al. 2012, WISE Explanatory Supplement, http://wise2.ipac.caltech.edu/docs/release/allwise/expsup/ index.html

Dallacasa, D., Stanghellini, C., Centonza, M., \& Fanti, R. 2000, A\&A, 363,887

Dasyra, K. M., \& Combes, F. 2012, A\&A, 541, L7

Dasyra, K. M., Combes, F., Novak, G. S., et al. 2014, A\&A, 565, 46

De Breuck, C., Seymour, N., Stern, D. J, et al. 2010, ApJ, 725, 36

Delvecchio, I., Lutz, D., Berta, S., et al. 2015, MNRAS, 449, 373

Dey, A., Soifer, B. T., Desai, V., et al. 2008, ApJ, 677, 943

De Young, D. 2010, ApJ, 710, 743

Dicken, D., Tadhunter, C., Axon, D., et al. 2011, ApJ, 741, 126

Dowell, C. D., Allen, C. A., Babu, R. S., et al. 2003, Proc. SPIE, 4855, 73

Downes, D., \& Solomon, P. 1998, ApJ, 507, 615

Efstathiou, A. 2006, MNRAS, 371, L70

Efstathiou, A., Christopher, N., Verma, A., \& Siebenmorgen, R. 2013 MNRAS, 436, 1873

Efstathiou, A., \& Rowan-Robinson, M. 1995, MNRAS, 273, 649

Efstathiou, A., Rowan-Robinson, M., \& Siebenmorgen, R. 2000, MNRAS, 313,734

Efstathiou, A., \& Siebenmorgen, R. 2009, A\&A, 502, 541

Eisenhardt, P. R. M., Wu, J., Tsai, C.-W., et al. 2012, ApJ, 755, 173 (EWB12) Elitzur, M. 2012, ApJL, 747, L33

Elvis, M., Wilkes, B. J., McDowell, J. C. R., et al. 1994, ApJS, 95, 1

Fabian, A. 2010, ARA\&A, 50, 455

Farrah, D., Bernard-Salas, J., Spoon, H. W. W., et al. 2007, ApJ, 667, 149

Feruglio, C., Fiore, F., Carniani, S., et al. 2015, in press

Feruglio, C., Maiolino, R., Pinconcelli, E., et al. 2010, A\&A, 518, L155

Fiolet, N., Omont, A., Polletta, M., et al. 2009, A\&A, 508, 117

Fischer, J., Sturm, E., Gonzalez-Alfonso, E., et al. 2010, A\&A, 518, L41

Fu, H., \& Stockton, A. 2007, ApJ, 666, 794

Garca-Burillo, S., Combes, F., Usero, A., et al. 2014, A\&A, 567, 125

Gonzalez-Alfonso, E., Fischer, J., Graci-Carpio, J., et al. 2014, A\&A, 561, 27

Griffin, M. J., Abreu, A., Ade, P. A. R., et al. 2010, A\&A, 518, L3

Grimes, J. A., Rawlings, S., \& Willox, C. J. 2005, MNRAS, 359, 1345

Guillard, P., Boulanger, F., Lehnert, M. D., et al. 2015, A\&A, 574, A32

Guillard, P., Ogle, P. M., Emonts, B. H. C., et al. 2012, ApJ, 747, 95

Heckman, T. M., \& Best, P. N. 2014, ARA\&A, 52, 589

Holt, J., Tadhunter, C. M., \& Morganti, R. 2008, AN, 387, 639

Ibar, E., Cirasuolo, M., Ivison, R., et al. 2008, MNRAS, 386, 953

Ivison, R. J., Chapman, S. C., Faber, S. M., et al. 2007, ApJ, 660, 77

Jarrett, T. H., Cohen, M., Masci, F., et al. 2011, ApJ, 735, 112

Jones, S., Blain, A. W., Lonsdale, C. J., et al. 2015, MNRAS, 448, 3325

Jones, S. F., Blain, A. W., Stern, D., et al. 2014, MNRAS, 443, 146

Kennicutt, R. C. 1998, ApJ, 498, 181

Kewley, L. J., Maier, C., Yabe, K., et al. 2013, ApJ, 774, 10

Kim, M., Ho, L. C., Lonsdale, C. J., et al. 2013, ApJL, 768, L9

Kormendy, J., \& Ho, L. C. 2013, ARA\&A, 51, 511

Lacy, M., Petric, A. O., Martinez-Sansigre, A., et al. 2011, AJ, 142, 196

Lacy, M., Ridgway, S. E., Sajina, A., et al. 2015, ApJ, 802, 102

Lanz, L., Ogle, P. M., Evans, D., et al. 2015, ApJ, 801, 17

Leitherer, C., \& Chen, J. 2010, NewA, 14, 356

Leroy, A. K., Walter, F., Bigiel, F., et al. 2009, AJ, 137, 4670

Lonsdale, C. J., Lonsdale, C. J., Smith, H. E., \& Diamond, P. 2003, ApJ, 592, 804

Lonsdale, C. J., Polletta, M., Omont, A., et al. 2009, ApJ, 692, 422

Lonsdale, C. J., Polletta, M., Surace, J., et al. 2004, ApJS, 154, 54

Lutz, D., Yan, L., Armus, L., et al. 2005, ApJL, 632, L13

Magnelli, B., Lutz, D., Santini, P., et al. 2012, A\&A, 539, 155

Maraston, C. 2005, MNRAS, 362, 799

Melbourne, J., Soifer, B. T., Desai, V., et al. 2012, AJ, 143, 125 
Morganti, R., Frieswijk, W., Oonk, R. J. B., Oosterloo, T., \& Tadhunter, C. 2013, A\&A, 552, L4

Morganti, R., Tadhunter, C. N., Oosterloo, T. A., et al. 2003, PASA, 20, 129 Mullaney, J. R., Alexander, D. M., Fine, S., et al. 2013, MNRAS, 433, 622

Mullaney, J. R., Alexander, D. M., Goulding, A. D., \& Hickox, R. C. 2011, MNRAS, 414, 1082

Nenkova, M., Sirocky, M. M., Ivezic, Z., \& Elitzur, M. 2008, ApJ, 685, 147 Nesvadba, N., Lehnert, M., De Breuck, C., Gilbert, A., \& van Breughel, W. 2008, A\&A, 479, 67

Nesvadba, N. H., Boulanger, F., Lehnert, M. D., Guillard, P., \& Salome, P. 2011a, A\&A, 536, L5

Nesvadba, N. P. H., Polletta, M., Lehnert, M. D., et al. 2011b, MNRAS, 415,2359

Netzer, H., Lutz, D., Schweitzer, M., et al. 2007, ApJ, 666, 806

O'Dea, C. 1998, PASP, 110, 493

Ogle, P., Boulanger, F., Guillard, P., et al. 2010, ApJ, 724, 1193

Pinconcelli, E., Vignali, C., Bianchi, S., et al. 2015, A\&A, 574, 9

Poglitsch, A., Waelkens, C., Geis, N., et al. 2010, A\&A, 518, 2

Polletta, M., Omont, A., Berta, S., et al. 2008, A\&A, 492, 81

Polletta, M., Tajer, M., Maraschi, L., et al. 2007, ApJ, 663, 81

Polletta, M., Wilkes, B. J., Siana, B., et al. 2006, ApJ, 642, 673

Priddey, R. S., Gallagher, S. C., Isaak, K. G., et al. 2007, MNRAS, 374, 867

Priddey, R. S., Isaak, K. G., McMahon, R. G., Robson, E. I., \& Pearson, C. P. 2003, MNRAS, 344, L74

Ramos-Almeida, C., Snchez-Portal, M., Prez Garca, A. M., et al. 2013, MNRAS, 417, 46

Richards, G. T., Lacy, M., Storrie-Lombardi, L. J., et al. 2006, ApJS, 166, 470

Sajina, A., Yan, L., Fadda, D., et al. 2012, ApJ, 757, 13

Scoville, N., Sheth, K., Walter, F., et al. 2015, ApJ, 800, 108
Scoville, N. Z., Aussel, H., Sheth, K. S., et al. 2014, ApJ, 783, 84 Seymour, N., Stern, D., De Breuck, C., et al. 2007, ApJS, 171, 353 Shang, Z., Brotherton, M. S., Wills, B. J., et al. 2011, ApJS, 196, 2 Shih, H.-Y., \& Stockton, A. S. 2014, ApJ, 794, 117

Silva, A., Sajina, A., Lonsdale, C., \& Lacy, M. 2015, ApJL, 806, L25

Simpson, C., Rawlings, S., Ivison, R., et al. 2012, MNRAS, 421, 3060

Stalevski, M., Fritz, J., Baes, M., Nakos, T., \& Popovic, L. 2012, MNRAS, 420, 2756

Stern, D., Assef, R. J., Benford, D. J., et al. 2012, ApJ, 753, 30

Stern, D., Lansbury, G. B., Assef, R. J., et al. 2014, ApJ, 794, 102

Sturm, E., Gonzalez-Alfonso, E., Veilleux, S., et al. 2011, ApJ, 733, 16

Tadhunter, C., Dicken, D., Morganti, R., et al. 2014a, MNRAS, 445, 51

Tadhunter, C., Holt, J., Gonzalez Delgado, R., et al. 2011, MNRAS, 412, 960

Tadhunter, C., Morganti, R., Rose, M., Oonk, J. B. R., \& Oosterloo, T. 2014b, Natur, 511, 440

Tsai, C.-W., Eisenhardt, P., Wu, J., et al. 2015, ApJ, 805, 90

Ulvestad, J. S., Wrobel, J. M., \& Carilli, C. L. 1999, ApJ, 516, 127

Veilleux, S., Melendez, M., Sturm, E., et al. 2013, ApJ, 776, 27

Wagner, A., \& Bicknell, G. 2011, ApJ, 728, 9

Wagner, A., Bicknell, G., \& Umemura, M. 2012, ApJ, 757, 136

Wagner, A., Umemura, M., \& Bicknell, G. 2013, ApJL, 763, L18

Wilson, C. D., Rangwala, N., Glenn, J., et al. 2014, ApJ, 789, 36

Wright, E. L., Eisnehardt, P., Mainzer, A. K., et al. 2010, AJ, 140, 1868

Wu, J., Bussmann, R. S., Tsai, C.-W., et al. 2014, ApJ, 793, 8

Wu, J. J., Tsai, C.-W., Sayers, J., et al. 2012, ApJ, 756, 96 (EWB12)

Yan, L., Chary, R., Armus, L., et al. 2005, ApJ, 628, 60

Yan, L., Donoso, E., Tsai, C.-W., et al. 2013, AJ, 145, 55

Zhang, K., Wang, T.-G., Yan, L., \& Dong, X.-B. 2013, ApJ, 768, 22 